University of Nebraska - Lincoln

DigitalCommons@University of Nebraska - Lincoln

Mechanical (and Materials) Engineering --

Dissertations, Theses, and Student Research

Mechanical \& Materials Engineering,

Department of

\title{
$5-2012$
}

\section{Material Handling System for Robotic Natural Orifice Surgery}

Jeff Midday

University of Nebraska-Lincoln, jeff.midday@yahoo.com

Follow this and additional works at: https://digitalcommons.unl.edu/mechengdiss

Part of the Mechanical Engineering Commons

Midday, Jeff, "Material Handling System for Robotic Natural Orifice Surgery" (2012). Mechanical (and

Materials) Engineering -- Dissertations, Theses, and Student Research. 30.

https://digitalcommons.unl.edu/mechengdiss/30

This Article is brought to you for free and open access by the Mechanical \& Materials Engineering, Department of at DigitalCommons@University of Nebraska - Lincoln. It has been accepted for inclusion in Mechanical (and Materials) Engineering -- Dissertations, Theses, and Student Research by an authorized administrator of DigitalCommons@University of Nebraska - Lincoln. 


\title{
MATERIAL HANDLING SYSTEM FOR ROBOTIC NATURAL ORIFICE SURGERY
}

by

Jeff Midday

\section{A THESIS}

\author{
Presented to the Faculty of \\ The Graduate College at the University of Nebraska \\ In Partial Fulfillment of Requirements \\ For the Degree of Master of Science
}

Major: Mechanical Engineering

Under the Supervision of Professor Carl A. Nelson

Lincoln, Nebraska

May, 2012 


\title{
MATERIAL HANDLING SYSTEM FOR ROBOTIC NATURAL ORIFICE SURGERY
}

\author{
Jeff Midday, M.S.
}

University of Nebraska, 2012

Advisor: Carl A. Nelson

Natural Orifice Translumenal Endoscopic Surgery (NOTES) is a relatively new surgical approach which uses no external incisions, thereby improving cosmetic outcomes, decreasing overall recovery time and reducing the risk of external infection. In standard NOTES, flexible endoscopic tools have been used to carry out a variety of surgical procedures in the abdomen. As an alternative, miniature in vivo robots can be fully inserted into the peritoneal cavity and utilized to perform various surgical procedures. These in vivo robots eliminate tool triangulation issues, improve multitasking capabilities and greatly increase freedom and dexterity when compared to standard endoscopic and laparoscopic tools. One major limitation is that once inserted, the in vivo robots are isolated within the abdomen and cannot send or receive materials to the external environment. The focus of this thesis is a Material Handling System (MHS) that has been developed to bridge this deficiency.

This system features a flexible silicone overtube and an open-loop control system with manual and automatic operation capabilities. The system utilizes the helix of a spring to advance a payload along the length of the overtube. All of the design rationale, design decisions, components and materials are discussed. Additional description of all of the electronic hardware, coupled with the programming logic, provides detailed insight into the open-loop control strategy. The bench-top and in vivo testing results of the completed device are presented. 
This thesis also addresses finite element modeling of the dimensional changes of silicone tubing under bending. The model looks at the complex issue of modeling a continuum rubber such as silicone, validated experimentally. The model provides general guidelines for the bending and kinking properties of a wide variety of tubing diameters and thicknesses. This tubing model can increase an engineer's ability to properly dimension and tolerance an overtube, such as that found in the MHS, based on the bending criteria of the device. 


\section{Acknowledgements}

Special thanks to Professor Carl A. Nelson for his assistance throughout the development of this device and the preparation of this manuscript. Thank you to Dmitry Oleynikov and others from the UNMC Center for Advanced Surgical Technology for your assistance conducting in vivo testing. Thank you also to Alan Goyzueta and all of the other researchers in the Applied Mechanisms and Design Research Lab for your contributions to this research. Finally, I would like to extend a big thank you to my family for supporting me throughout my educational pursuits. 


\section{Table of Contents}

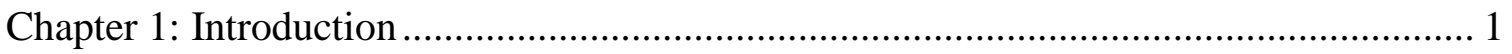

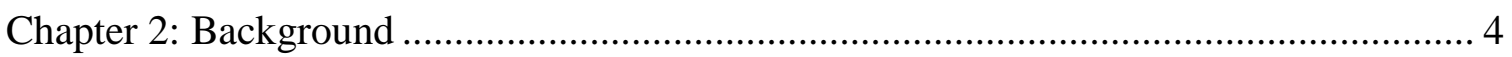

2.1 MIS: Laparoscopy, LESS \& NOTES.............................................................. 4

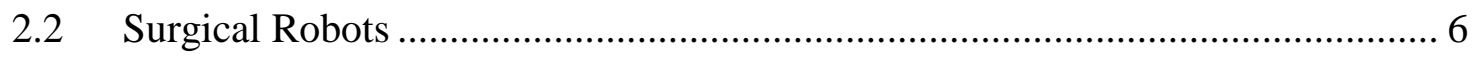

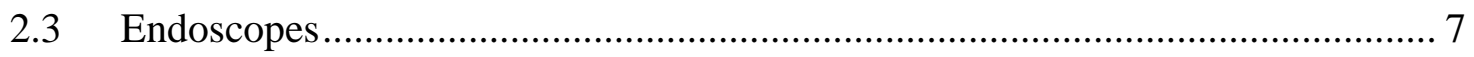

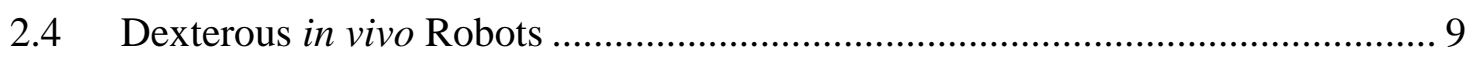

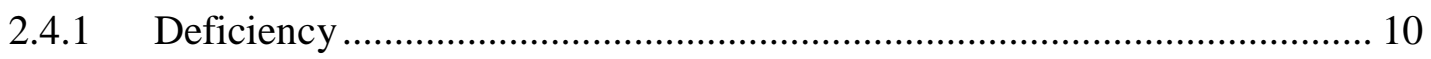

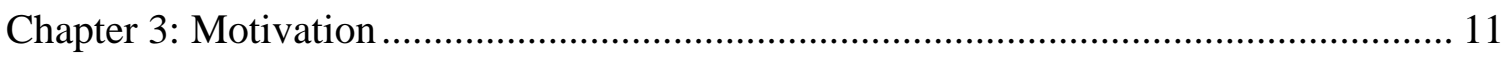

Chapter 4: Physical Design - Insertion Assembly........................................................... 13

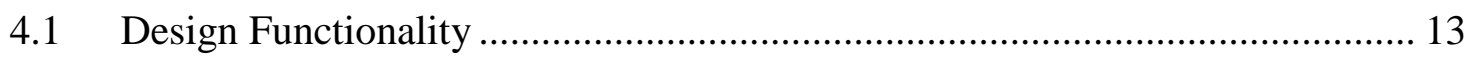

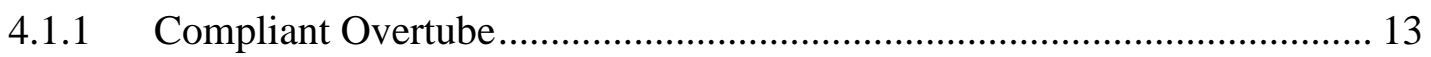

4.1.2 Material Capture Device ................................................................ 15

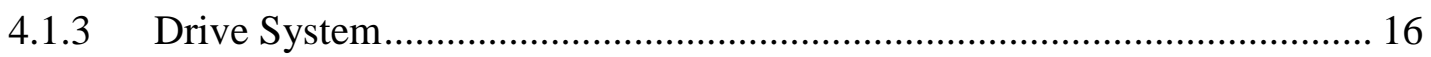

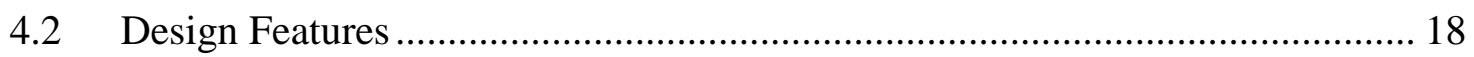

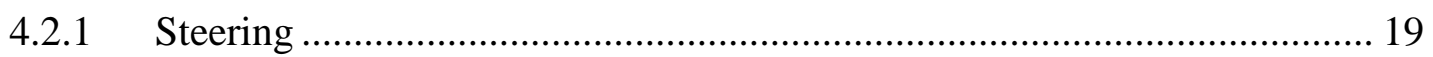

4.2.2 Lighting and Video ………………………….................................... 21

4.2.3 Suction and Irrigation ......................................................................... 21

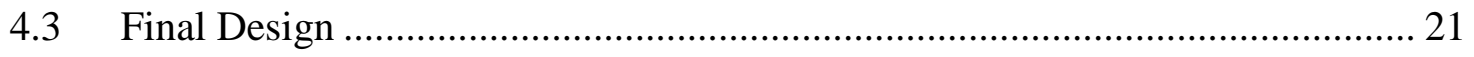

4.4 Insertion Assembly - Other Details .............................................................. 22

4.5 Special Manufacturing Considerations ............................................................ 25

Chapter 5: Electronics and Control System ................................................................ 26

5.1 Microprocessor and Motor Driver................................................................ 26

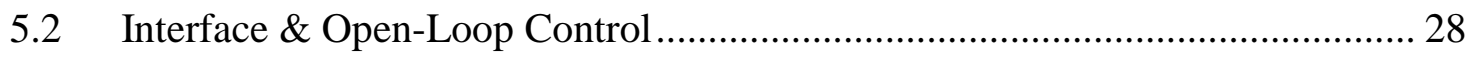

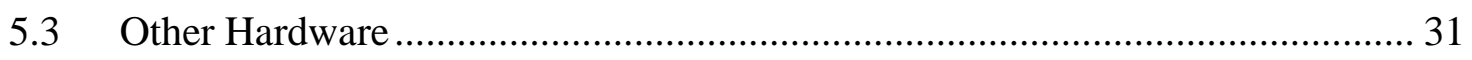

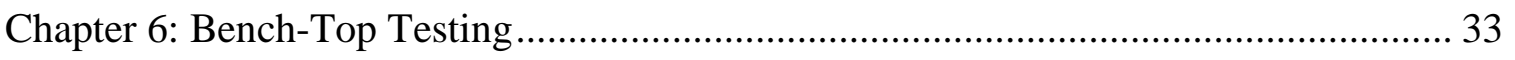

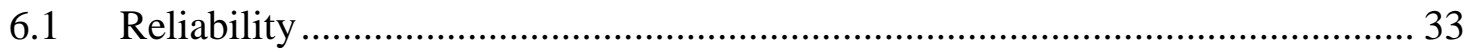

6.2 Maxima: Bend Radius \& Axial Twist............................................................. 34

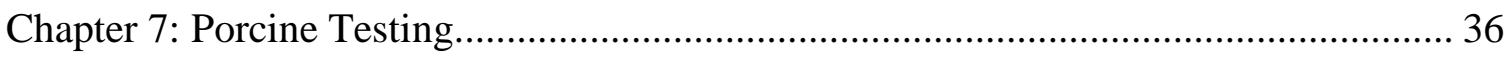

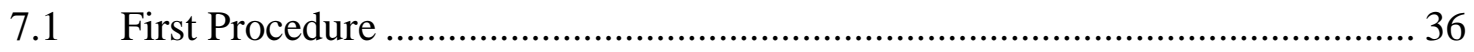

7.2 Improvements \& Second Procedure.................................................................. 38

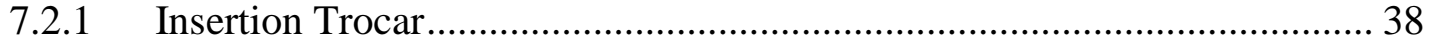

7.2.2 Second Procedure................................................................................... 40 
Chapter 8: FE Analysis of Silicone Tubing Under Bending.............................................. 44

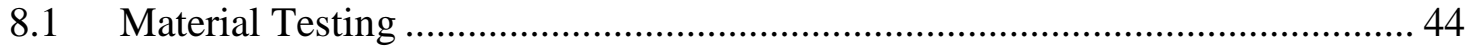

8.2 Material Model Verification....................................................................... 48

8.3 Finite Element Simulation............................................................................ 50

8.3.1 Bend Radius Evaluation....................................................................... 52

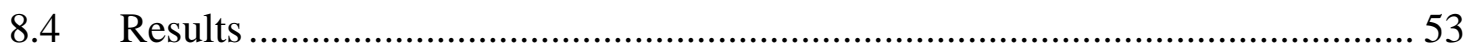

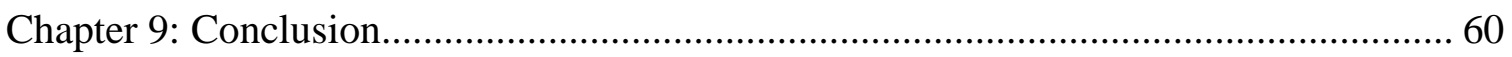

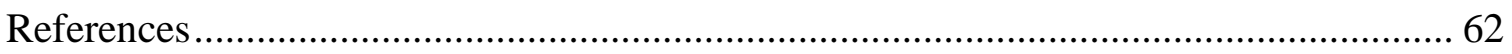

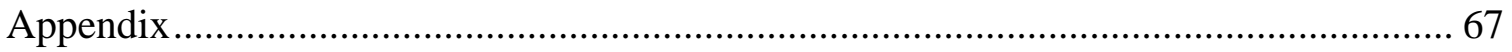

Appendix A: MHS Insertion w/ Articulated Endoscope ............................................... 67

Appendix B: MHS Start-up Procedure \& Control ....................................................... 69

Appendix C: Important Datasheets ....................................................................... 71

C.1 Low-Friction Coating for Silicone Tubing................................................... 71

C.2 Stepper Motor ......................................................................................... 72

C.3 Stepper Motor Encoder............................................................................... 74

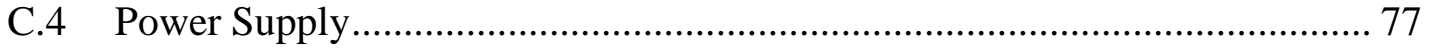

Appendix D: Control Logic \& Programming ........................................................... 79

Appendix E: LS-Dyna - Deck Code........................................................................ 85

Appendix F: Matlab FEA Processing Code................................................................ 89

Appendix G: Bill of Materials ............................................................................... 90

Appendix H: Assembly Drawings ........................................................................... 93

Appendix I: Component Drawings ............................................................................... 96 


\section{List of Figures}

Figure 1-1: NOTES Material Handling System........................................................... 3

Figure 2-1: Laparoscopic abdominal surgery - external setup [2-5] .............................. 4

Figure 2-2: Transumbilical laparoendoscopic single-site surgery [2-7] .......................... 5

Figure 2-3: Tract for NOTES transgastric approach.................................................... 6

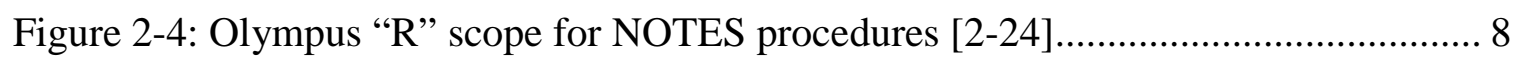

Figure 2-5: In vivo robots [2-30], [2-31] ( (C [2008], [2006] IEEE) .................................. 9

Figure 4-1: Overtube selection array, top to bottom: silicone, braided vinyl, plain vinyl,

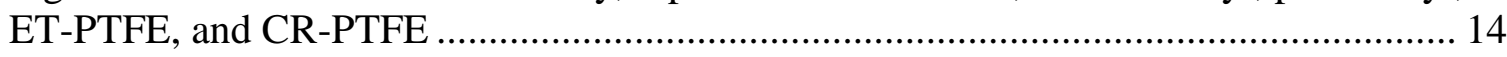

Figure 4-2: Passive Nitinol spring grasper (light lines approximate deflected contour) .. 15 Figure 4-3: Shuttle/overtube geometry (A) Offset tab configuration, (B) Shuttle tab

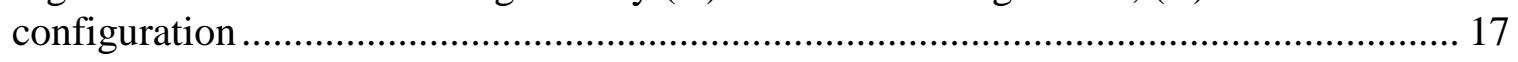

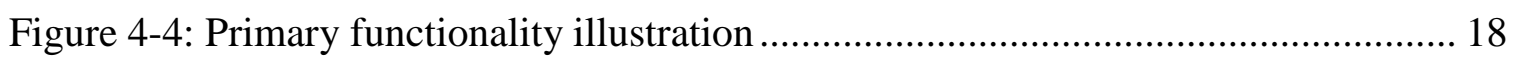

Figure 4-5: Comparison of round vs. non-round cross-sections .................................... 19

Figure 4-6: Standard endoscope and articulated fiberscope inserted into the overtube ... 20

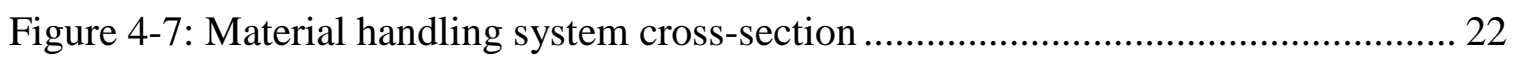

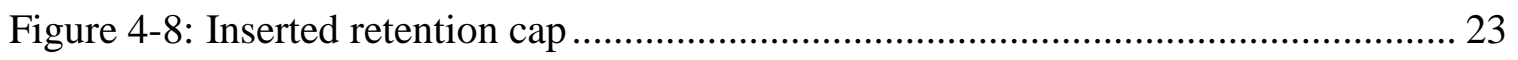

Figure 4-9: Distribution cap. (1) overtube, (2) grip pad, (3) motor coupling, (4) rotary insufflation seal, (5) magnetic attachment base, (6) fiberscope outlet, (7) 4mm quick

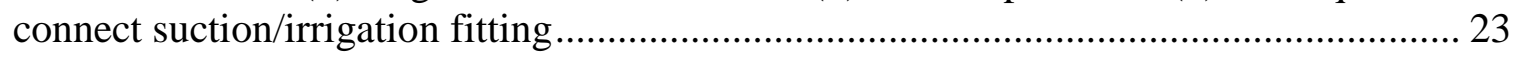

Figure 4-10: Magnetic attachment point for the distribution cap ................................. 24

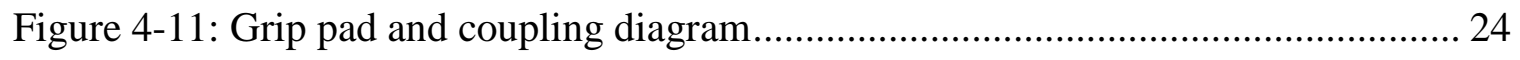

Figure 4-12: (A) Horizontal extrusion droop; (B) Accurate vertical extrusion ................ 25

Figure 5-1: FEZ Panda II (left), Ruggeduino (right) ................................................. 26

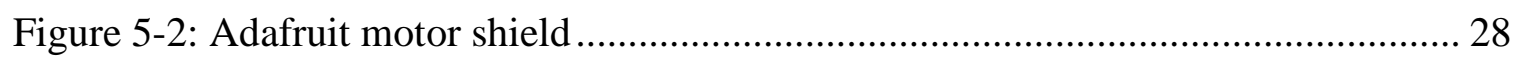

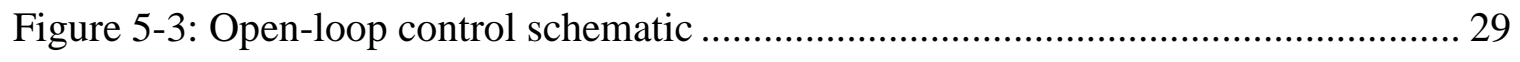

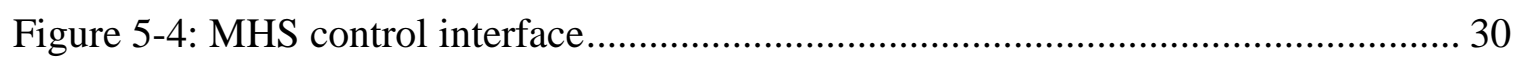

Figure 5-5: Debounce circuit diagram (left), implemented circuit (right) ....................... 31

Figure 5-6: Completely wired electronics package .................................................... 32

Figure 6-1: Repeatability test: $12.7 \mathrm{~cm}$ bend radius, $180^{\circ}$ axial twist test ...................... 34

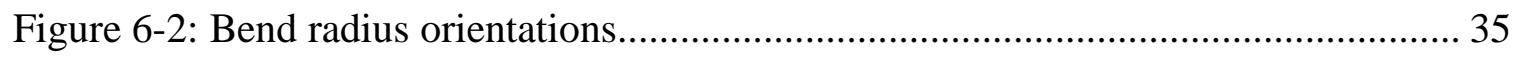

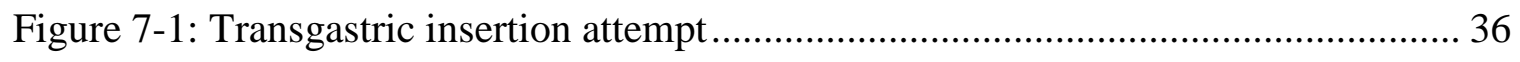

Figure 7-2: System inserted in vivo via gel port ..................................................... 37

Figure 7-3: A typical trocar configuration, listed by the FDA [7-1] .............................. 38

Figure 7-4: MHS trocar in the retracted (top) and extended (bottom) configurations...... 39 


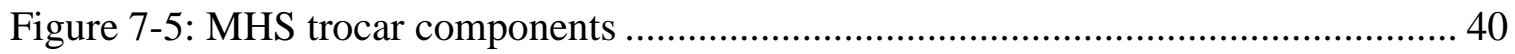

Figure 7-6: Transvaginal trocar insertion: exterior (left), interior (right) ....................... 41

Figure 7-7: Trocar piercing assembly removed from cannula...................................... 41

Figure 7-8: Insertion of MHS into trocar cannula: exterior (left), interior (right) ........... 42

Figure 7-9: Raised abdominal wall with inserted MHS, indicating proper insufflation... 42

Figure 7-10: Fully inserted shuttle, dyed blue for proper visualization.......................... 43

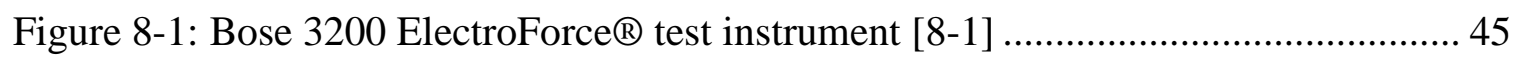

Figure 8-2: Young's modulus: engineering stress vs. strain ........................................ 45

Figure 8-3: Longitudinal wave transmission and reflections ( $2 \mu$ s divisions) ................... 46

Figure 8-4: Single element pull test load vs. deflection comparison.............................. 49

Figure 8-5: Modified shear modulus load/deflection curve comparison ......................... 50

Figure 8-6: Shell element silicone tubing model ....................................................... 51

Figure 8-7: Example of the circle-fit function through points on the neutral axis .......... 53

Figure 8-8: Tubing bending modes: ripple, a single kink and total collapse (left to right)

Figure 8-9: Kink point moving asymptotically toward the fixed end............................ 54

Figure 8-10: Bend radius/parameter relationships ..................................................... 56

Figure 8-11: Bend radius as a function of non-dimensional diameter/thickness ............. 57

Figure 8-12: Illustration of the dimension change in the plane cross section.................. 57

Figure 8-13: Dimensional Changes with respect to instantaneous bend radius................ 58

Figure 8-14: Stress distribution comparison between different bending modes.............. 59

\section{List of Tables}

Table 8-1: Parameter combinations for the silicone rubber FE study ............................. 51

Table 8-2: Bending mode behavior for all models ...................................................... 55 


\section{Chapter 1: Introduction}

In decades past, almost all surgical procedures were performed through "open” approaches. The surgical operating theatre has seen a paradigm shift from open procedures to a combination of laparoscopic and endoscopic minimally invasive surgery (MIS) techniques. The promise of reduced recovery time and decreased risk for infection has turned laparoscopic surgery into the new standard in healthcare. Additionally, endoscopic medical procedures have been in use for several decades for a number of procedures such as foreign object removal, ultrasonic imaging and injection therapy. More recently, endoscopy has also been adapted to perform increasingly advanced surgical procedures. These MIS procedures do have some limitations: reduced instrument maneuverability due to the constraint of surgical access points, difficulty maintaining proper visual orientation, and when using multiple tools in concert, difficult triangulation (accessing the surgical site from different angles).

An alternative to these MIS procedures is Natural Orifice Translumenal Endoscopic Surgery (NOTES). NOTES does not require any external incisions, but rather passes surgical instrumentation through a natural orifice such as the esophagus and into the abdominal cavity. This natural access point further decreases the risk of infection, completely eliminates visible scarring, and expedites the recovery process. The downside of NOTES, like traditional endoscopy, involves the spatial and tool triangulation issues associated with the kinematic constraints imposed by the access method. 
To eliminate the spatial constraints, a novel use of robotics can be employed. Miniature in vivo robots can be inserted via a natural orifice access point, and once introduced into the abdominal cavity, have the freedom and dexterity to navigate the entire region uninhibited by the access point. Fully inserting in vivo robots into the body introduces a limitation as they are physically isolated from the surgeons performing the procedure.

This thesis presents a novel device to eliminate the miniature in vivo robots' isolation from the surgeons and the external environment. The device, a Material Handling System (MHS) shown in Figure 1-1, which closely resembles a traditional therapeutic endoluminal endoscope, is inserted via the same natural orifice access point as the in vivo robots. The MHS remains in the natural orifice for the duration of a surgical procedure. The device has a primary working channel for securing and transporting payloads between the robots and the external environment. The MHS is also equipped with secondary and tertiary lumens through which additional features, including steering, lighting, video access, suction and irrigation, can be provided.

The MHS utilizes a flexible silicone rubber overtube. Like most soft rubber tubing, the dimensions of silicone rubber tubing change during flexure. This property becomes a driving factor when selecting proper geometry and clearances for components which operate within the overtube. For this reason, this thesis also presents the development of a finite element (FE) model of a cylindrical silicone tube. 


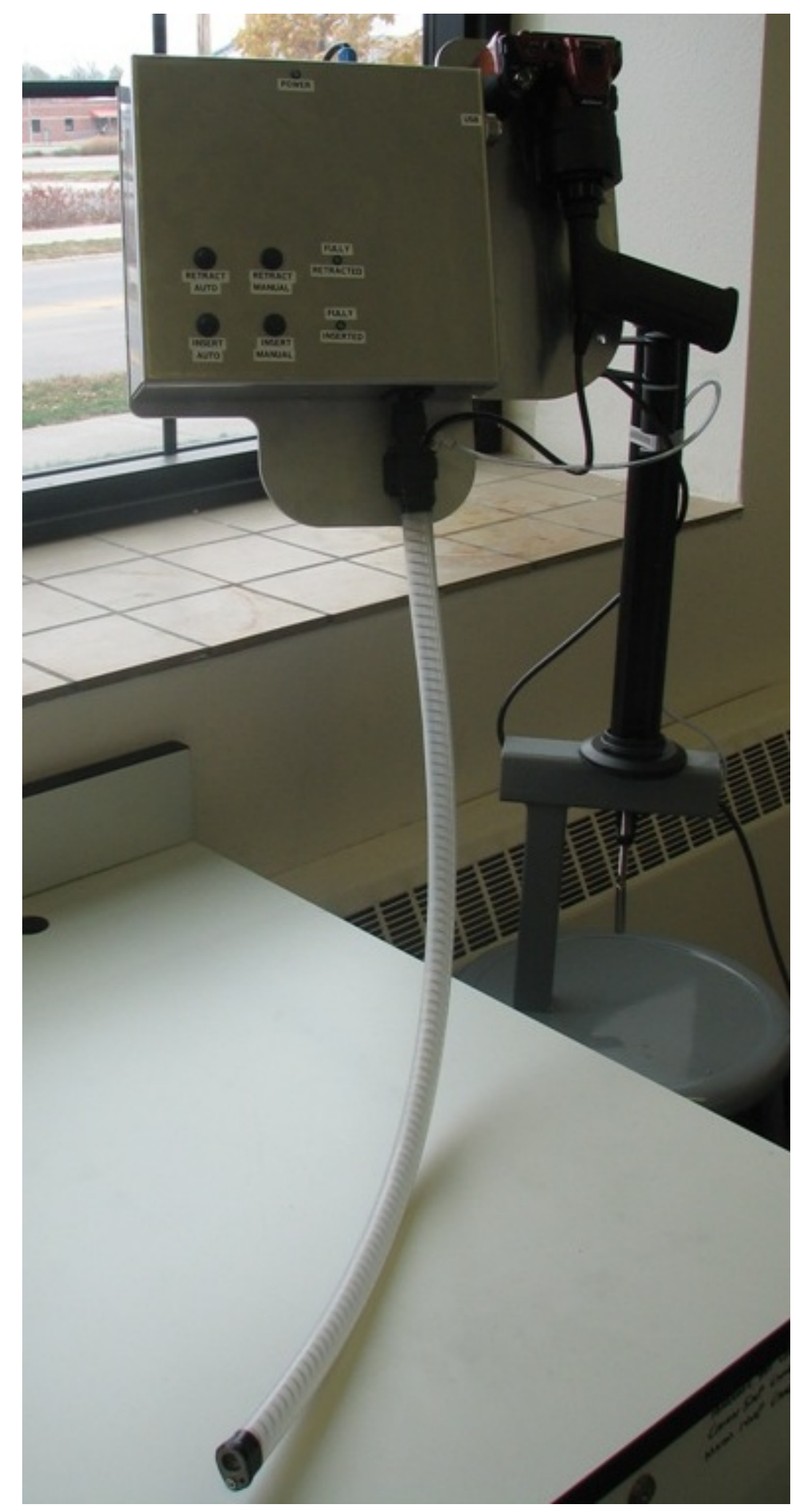

Figure 1-1: NOTES Material Handling System 


\section{Chapter 2: Background}

\subsection{MIS: Laparoscopy, LESS \& NOTES}

Minimally invasive surgery (MIS) is a broad label applied to any procedure that is done through a small incision or no incision at all. The first MIS procedure on a human was performed in 1910 by Hans Christian Jacobaeus [2-1], but this method was not widely adopted until the 1990s [2-2, 2-3]. MIS offers a large number of patient benefits, including reduced risk of infection, smaller incisions, less scarring and faster recovery times [2-4]. MIS presents a large number of challenges for a surgeon including poor depth perception, difficulty orienting and triangulating instruments, and spatial limitations introduced by the constraint of the access point.

Traditional laparoscopic surgery involves making a series of small incisions for multiple tools to pass into the abdominal cavity, as shown in Figure 2-1. Tools are usually passed through trocars, surgical instruments with a cutting point enclosed in a tube, or a gel port. The abdominal cavity is "insufflated" with low-pressure carbon dioxide to lift the abdominal wall above the organs, making room for the surgical instruments and allowing for visualization of the surgical field.

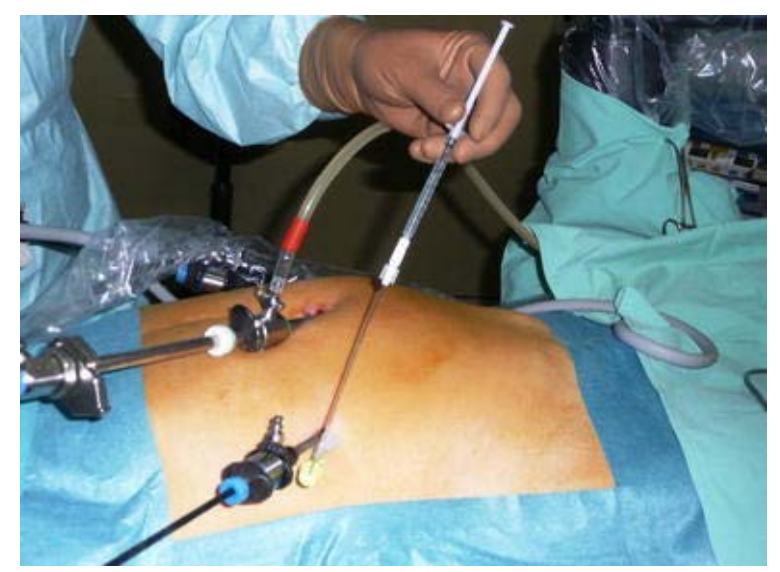

Figure 2-1: Laparoscopic abdominal surgery - external setup [2-5] 
Laparoendoscopic Single-Site (LESS) surgery is the next evolution of MIS procedures. LESS has been proven to reduce operation time and has comparable complications to traditional laparoscopy through a number of procedures such as cholecystectomies and appendectomies [2-6]. LESS surgical procedures present a unique challenge in that the instrumentation is crossed at the point of entry, making the external right-hand instrument the left instrument internally and vice versa [2-7]. An external view of a transumbilical LESS procedure is shown in Figure 2-2.

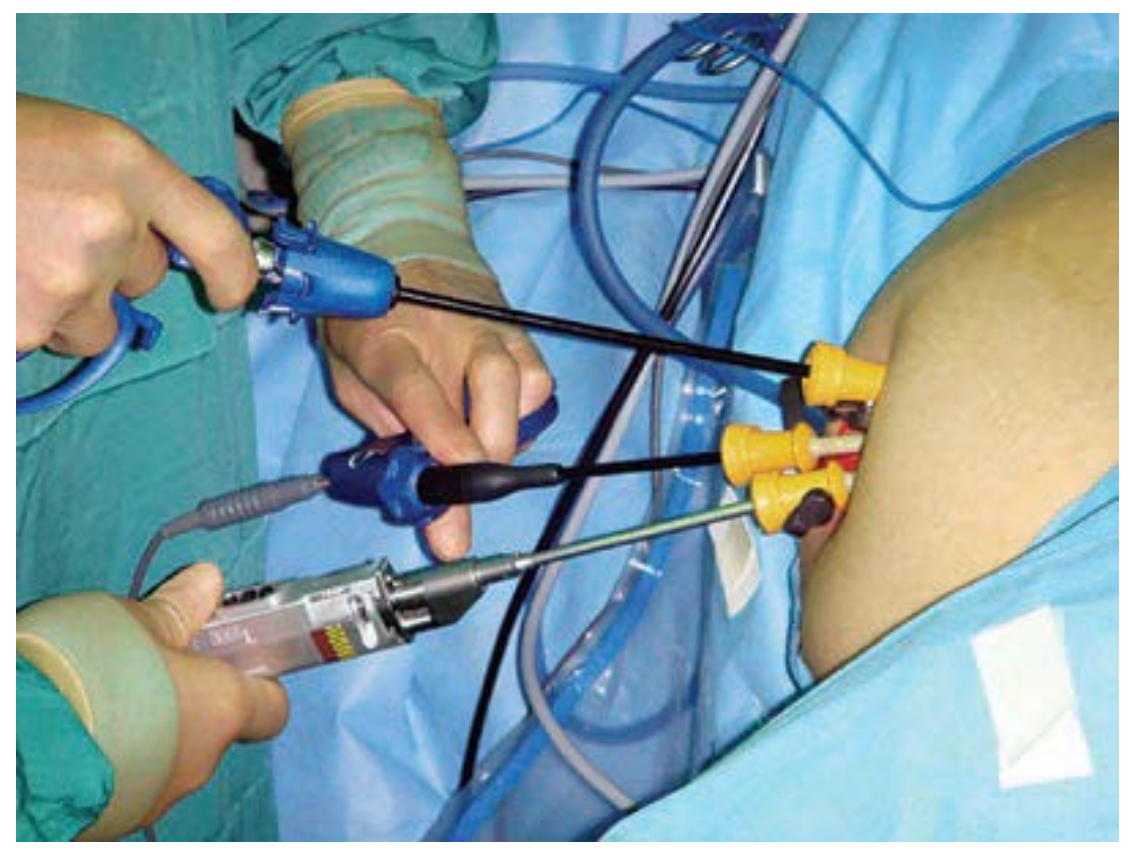

Figure 2-2: Transumbilical laparoendoscopic single-site surgery [2-7]

Natural Orifice Translumenal Endoscopic Surgery (NOTES) is the next level of decreased invasiveness in the MIS category. Traditional endoscopic surgery utilizes flexible, articulated instruments introduced through canals (working channels) in an endoscope to perform a variety of procedures, mainly in the peritoneal cavity [2-8]. NOTES builds off this basic endoscopic platform, and often uses a transgastric approach to gain entry to the abdominal cavity (Figure 2-3). 


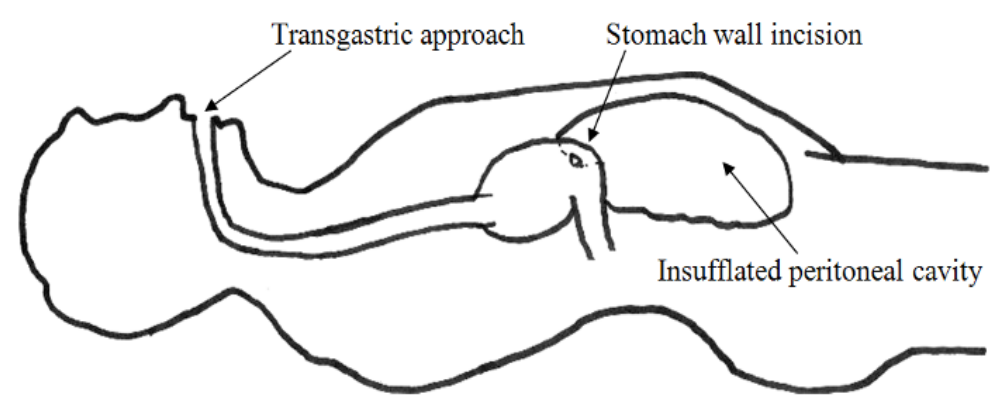

Figure 2-3: Tract for NOTES transgastric approach

NOTES eliminates the need for external incisions, vastly reducing the risk of external infection and expediting recovery [2-9]. NOTES is also advantageous compared to other MIS techniques because most organs are best accessed from a translumenal approach [2-10]. NOTES has been proven with successful survival animal surgeries [211] and Rao et al. performed the first NOTES procedure on a human [2-12]. The natural orifice access points, while having many patient benefits, present challenges for introducing instrumentation through the restrictive lumen size. Additionally, when using multiple tools, as in laparoscopy [2-13], tool triangulation can be difficult. Currently, however, the main downside to the approach is the lack of enabling technology [2-14].

\subsection{Surgical Robots}

Robotics has been moving rapidly to the forefront of the surgical world. There have been several laparoscopic robots approved by the FDA, including AESOP, da Vinci ${ }^{\circledR}$ and Zeus. The Automated Endoscopic System for Optimal Positioning (AESOP) is a camera control robot for laparoscopic procedures, but had little other functionality [215:2-17]. The da Vinci ${ }^{\circledR}$ system, created by Intuitive Surgical, is a cable-driven system with disposable tools capable of performing many laparoscopic procedures. The da Vinci ${ }^{\circledR}$ can also be operated via telepresence, as it provides a stereoscopic image of the patient and the workspace to the surgeon operating the robot and the control consoles can 
be networked [2-18]. The Zeus system, created by Computer Motion, is a remotely operated system like the da Vinci ${ }^{\circledR}$, but uses robotic arms that mimic the surgeon, and it does not have the same telepresence capability. These surgical systems have proven very effective in MIS [2-19], but lack dexterity to perform NOTES procedures.

\section{$2.3 \quad$ Endoscopes}

Dexterity is necessary for natural orifice procedures because of the need for unusual orientation of surgical tools [2-20]. Currently for NOTES, there are a number of non-robotic endoscopic devices on the market, most notably the Transport and the Cobra created by USGI Medical and Swanstrom et al. [2-21]. The Transport utilizes four large access channels and a 4-way steering tip. Most endoscopes have triangulation issues [222], and the Cobra aims to eliminate these issues by using three independent arms with fixed instrumentation. It has not been widely successful due to the imprecise control and time consuming nature of changing the instruments [2-23].

There are also several endoscopic based robotic platforms that are commercially available. Olympus designed the "R" scope specifically for NOTES procedures, shown in Figure 2-4. The device is similar to a therapeutic scope and has channels for suction, irrigation and for instruments to pass through. The tip is also dual-articulated, meaning it can bend in two separate directions, and the tip can also move freely when the rest of the body is locked. The device is difficult to control, is visually disorienting, and generates less-than-acceptable force levels to operate properly [2-24]. 


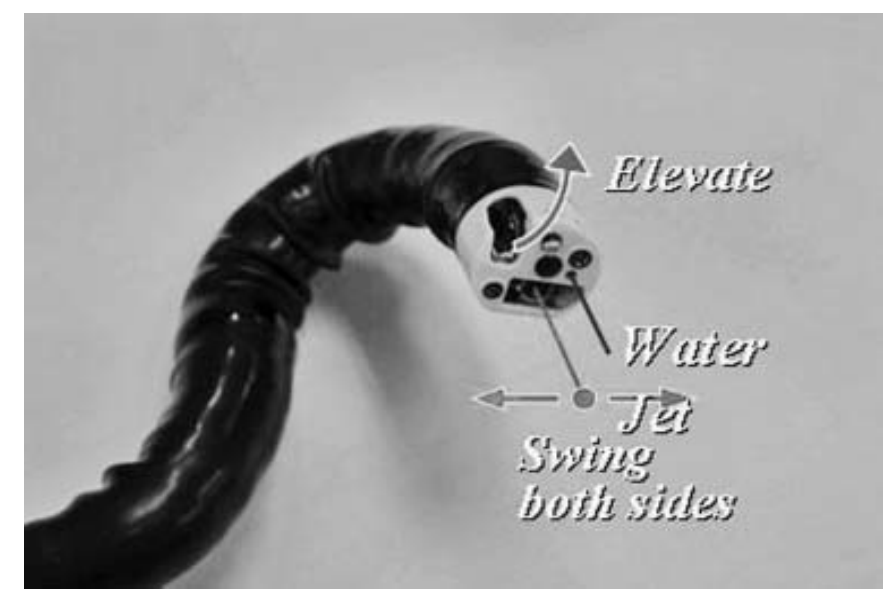

Figure 2-4: Olympus “R” scope for NOTES procedures [2-24]

The ViaCath System is a commercially available device that utilizes haptic feedback, and is set up in a master-slave configuration [2-25]. Both the ViaCath and the "R" scope are cable driven, which is reported by Lirici et al. to be too imprecise [2-26]. Other control mechanisms have been developed - a tendon and sheath configuration by Phee [2-27], and a rigid cylindrical link system by Ota [2-28]. The tendon and sheath method proved to have delay and hysteresis during operation, and the cylindrical link system provided larger force capability, but was still actuated by cable.

The multifunctional articulating surgical robot, developed by Nelson et al. [2-29], utilizes a single end-effector arm that is attached to a steerable and shape lockable drive system. The robot is equipped with a rotary tool changer, enabling up to three different tools to be utilized during a procedure, without ever removing the device from the natural access point. The aim of the device is to provide a dexterous robotic platform that can supply improved force transmission compared to the other commercially available NOTES endoscopes. All of these endoscopic platforms are dexterous enough (i.e., have enough degrees of freedom) to perform NOTES procedures, but platform stability, force generation, triangulation difficulty and accuracy issues are all still present. 


\subsection{Dexterous in vivo Robots}

An alternative to traditional endoscope-based techniques is using in vivo miniature robots for NOTES. These in vivo robots can be fully introduced into the peritoneal cavity via a transgastric, transvaginal or transcolonic approach [2-30]. Several examples of in vivo robots (Figure 2-5) have been developed and tested on porcine models. [2-30:2-37].

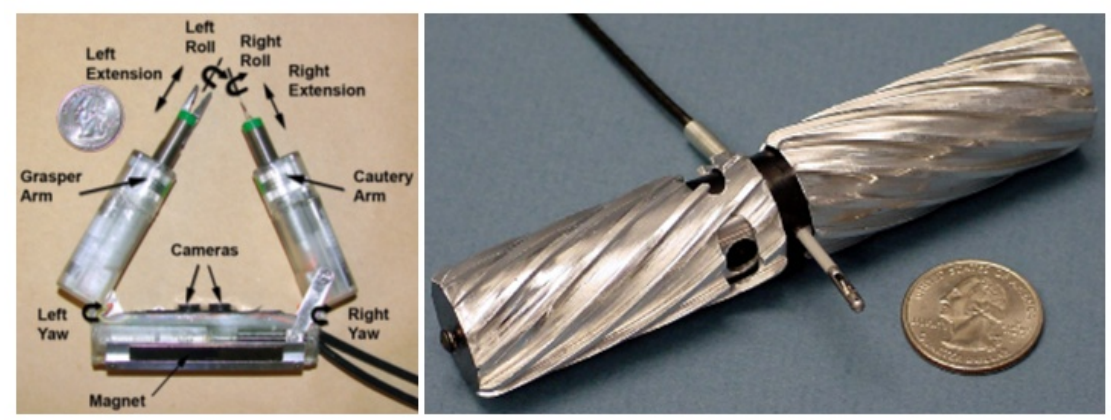

Figure 2-5: In vivo robots [2-30], [2-31] (@ [2008], [2006] IEEE)

One early example, developed by Lehman et al., is a stereoscopic two-armed dexterous robot [2-30]. The arms can be folded flat during insertion, and can be reoriented once situated in the abdominal cavity. The robot is attached to the upper interior abdominal wall via an external magnet system. The robot can be maneuvered around the insufflated abdominal cavity via the external magnet. The robot was originally intended for vision and task assistance during laparoscopic procedures [2-32]. Further iterations of the design showed the feasibility to apply significant forces and can be operated in multiple orientations [2-33]. Dumpert et al. outfitted the system with a proportionalintegral (PI) controller and a vision system to allow semi-autonomous tasks to be completed [2-34]. The main limitation is that manual manipulation of the robot is required to move its limited workspace to a desired location. 
Another type of miniature robotic platform is the mobile, wheeled in vivo robots originally developed by Rentschler et al. [2-35]. These robots are long and slender, consist of one tool and are equipped with their own on-board camera. The robot is capable of producing large mechanical forces and is capable of biopsying hepatic tissue. Further iterations of the robot were designed by Hawks et al. and included advancements such as wireless operation, and require no external systems other than the surgical interface [2-36]. These robots were also proven to be useful in cooperative applications, with each robot being equipped with a different tool to accomplish a singular task [2-37].

\subsubsection{Deficiency}

Once inserted into the body, these in vivo robots have significantly more freedom and flexibility, as space constraints and tool triangulation issues are drastically reduced in the insufflated abdominal cavity. Fully inserting in vivo robots into the body introduces a new limitation as they are physically isolated from the surgeons performing the procedure. This limitation has not yet been addressed. 


\section{Chapter 3: Motivation}

The miniature mobile in vivo robots have shown a lot of promise for NOTES procedures and have been embodied in a number of forms. These robots all share the same affliction that once inserted they are isolated from the outside environment. In the case of a biopsy robot, once the tissue has been excised, it may be desirable for the robot to continue working; in that case the tissue currently being held needs to be transported out of the body, but there are no external incisions to reach in and grab it. In the case of a multifunctional robot that is capable of using a number of different tools, it may be desirable for new tools to be transported near the surgical site and oriented properly so the robot can grab them. In the case of a suturing robot, if more staples or sutures are required than are located on board the robot, a means is needed by which more material can be provided.

Current technology suggests that a simple therapeutic endoscope with a grasper could be passed through a natural orifice, e.g., esophagus, vagina, or colon, and sent to administer or receive a payload. This approach is flawed in that each time the scope is passed into and out of the natural orifice, more patient trauma is induced, and operation time is increased. An alternative method might be to insert all of the tools, sutures, staples, etc. that the robot might need throughout an operation at the beginning of the procedure. All removed tissues could be placed in refuse bags, and then once the procedure is complete, all materials could be removed at once. The flaw with this approach is that there is limited space within the peritoneal cavity, and crowding it with unnecessary supplies would only hinder a procedure’s speed and effectiveness. Another 
issue is that remnants could potentially be left behind, which poses a serious risk for postoperative infection and other complications.

A new technology is needed that can move any required payload between the in vivo robots and the outside environment while minimizing patient trauma and risk of infection, and reducing the amount of equipment needed inside the abdominal cavity. This thesis presents a device, a Material Handling System (MHS) which is inserted via the same natural access point as the in vivo robots, and remains in the orifice for the duration of a procedure. The primary functionality of the device is to secure and transport payloads through a working channel within an overtube. The MHS is also equipped with features including steering capability for insertion, lighting, video access, suction and irrigation. 


\section{Chapter 4: Physical Design - Insertion Assembly}

\subsection{Design Functionality}

The basis of designing any device for a NOTES procedure is the geometric constraints imposed by the natural orifice access point. Gaining entry into the abdominal cavity with a transvaginal approach is the most common method [4-1]; however, a transgastric approach is the most restrictive NOTES approach, and is applicable to both males and females; therefore the spatial design parameters are established from the anatomy of this tract. The average esophagus has a bend radius of $7.5 \mathrm{~cm}$ and generally an endoscope does not exceed $1 \mathrm{~m}$ in length. These dimensions allow a $25 \mathrm{~mm}$ diameter tube to be inserted along its length [4-2]. Based on esophageal constraints, the main functionality of the device is achieved with three distinct components: a compliant overtube, a material interface device and a drive system. These three coupled features provide the bridge between the in vivo robots and the surgical team.

\subsubsection{Compliant Overtube}

The first component of the Material Handling System is a compliant overtube, which is necessary to protect the patient from any trauma and provide adequate access to the peritoneal cavity. It also needs to be able to bend to the desired contours of the esophagus as well as make the necessary high-angle turn to navigate from the esophagus to the incision in the stomach wall to access the peritoneal cavity. Various types of PTFE, silicone and vinyl materials were initially screened (Figure 4-1) for their biocompatibility and for their varying magnitudes of friction and flexure properties. Simple, physical bend radius and overall compliance tests showed the PTFE and vinyl materials are not flexible enough to match the contours required for surgery. Silicone has 
the best combination of flexural properties and rigidity, and is also a commonly used biomaterial.

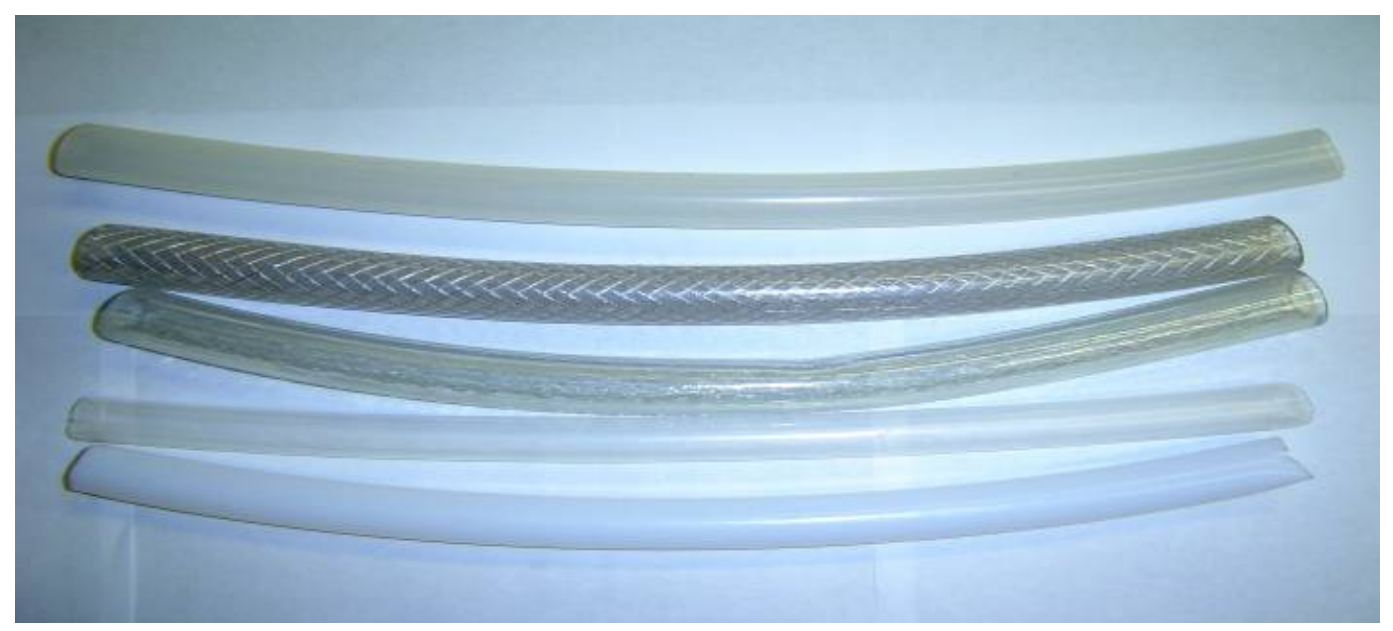

Figure 4-1: Overtube selection array, top to bottom: silicone, braided vinyl, plain vinyl, ET-PTFE, and CR-PTFE

The inherent issue with a silicone overtube and many other rubbery polymers is their high coefficient of friction. Depending on surface conditions, coefficients of friction greater than 3 are possible [4-3]. Both wet and dry lubricants may be applied to the surface of the silicone to reduce friction, thereby increasing the wear life of the system. It is desirable to use a more permanent dry coating, eliminating the need for reapplication. The typically selected dry coating is mechanically bonded Parylene, a rigid friction-reduction coating applied by vapor deposition. This treatment is expensive and usually cracks over time. Instead, a chemically bonded elastomeric coating, SlickSil ${ }^{\circledR}$ LSR (Surface Solutions Group, LLC), has been selected. This lower cost treatment is made specifically for medical silicones, reduces surface friction by approximately 50\% and also has anti-microbial properties; it is USP class IV certified for biocompatibility. 


\subsubsection{Material Capture Device}

A material interface to "shuttle" items between the miniature in vivo robots and the outside environment must be placed within the overtube. The interface must secure and properly orient the payload it is requested to carry. Possible materials the device may carry include various robotic tool tips, staples, refuse bags, and excised tissue. Many of the in vivo robots are still evolving, and tool tips such as cauteries, forceps and shears have varying geometries, so it is desirable to have a flexible platform to accept future generations of robotic tools. Passive compliant members are used to secure payload materials within the shuttle. These members are fabricated using a thin ribbon of a superelastic Shape Memory Alloy (SMA), Nitinol, shaped into a plateau-like profile (Figure 4-2) and run the length of the shuttle. The geometry of the "spring grasper" allows it to readily accept materials up to $4.8 \mathrm{~mm}$ in diameter and $20 \mathrm{~mm}$ long, so the shuttle can traverse the prescribed bend radius. Once the payload is fully inserted into the shuttle, the spring grasper provides a clamping force to secure the item.

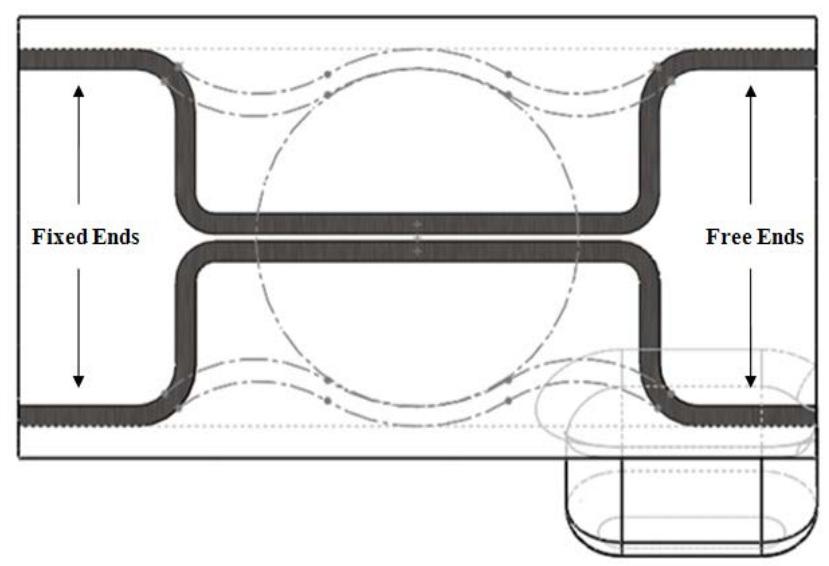

Figure 4-2: Passive Nitinol spring grasper (light lines approximate deflected contour) 


\subsubsection{Drive System}

Once material is placed in the spring grasper, it must traverse the length of the overtube. It is desirable that this operation be automated, and that it be as fast as possible, to minimize the impact on a procedure's duration, with the overall device being small in size (less than Ø25mm). A twist-tip mechanical pencil served as a source of inspiration for the design of this device. The material delivery system utilizes the helix of a spring to advance the shuttle, just as the pencil advances the pencil lead. As the spring rotates, the shuttle advances along the length of the overtube. The spring will be placed into the ID of the overtube, and will be longitudinally constrained so that it cannot translate, but only rotate on its longitudinal axis. The spring grasper is placed within a rigid "shuttle" which is concentrically positioned within the drive spring. For the shuttle to translate with respect to the overtube, it cannot be permitted to rotate.

To prevent the shuttle from rotating within the overtube, it must be mechanically constrained to the tubing. Initially an offset tab design to orient the shuttle with respect to the overtube (Figure 4-3A) was selected. Two tabs on the shuttle would contact opposing tabs on the tubing, thus restricting rotation. Silicone is a very difficult material to manufacture in any type of complex shape with marginal precision, and as a result, this idea was abandoned. After investigating manufacturing feasibility with several extrusion companies who specialized in medical silicone extrusions, adding a longitudinal groove or keyway slot to the tubing was a more feasible design. The shuttle has a protruding stud (Figure 4-3B) that fits between two coils of the drive spring and into the keyway in the overtube. 


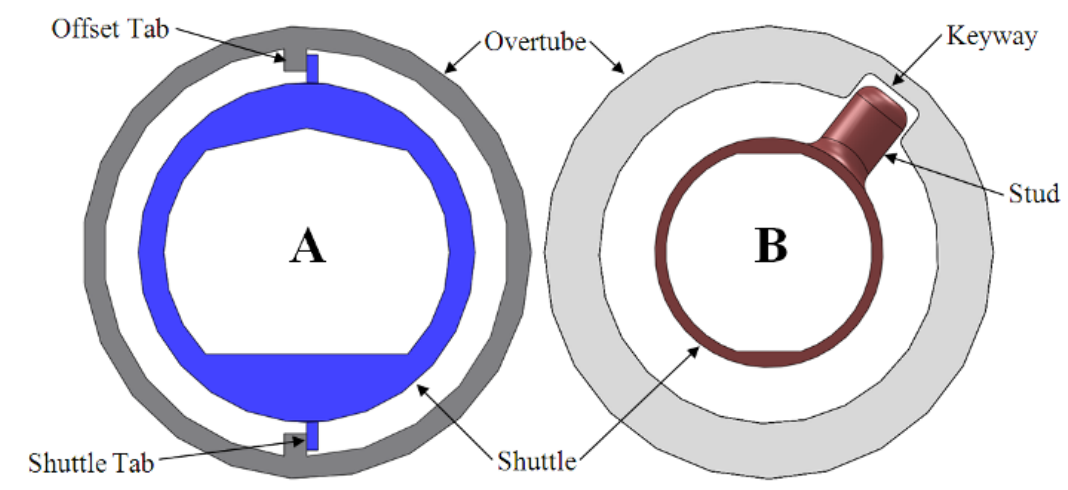

Figure 4-3: Shuttle/overtube geometry (A) Offset tab configuration, (B) Shuttle tab configuration

The shuttle, drive spring and overtube are all oriented concentrically, and use each other as bearing surfaces (Figure 4-4). The spring is made from $\varnothing 1.6 \mathrm{~mm} 316$ stainless steel, selected for its biocompatibility and relative strength. By controlling the number of coils per inch, and the rotation rate, friction can be minimized. If there are too many coils per inch, then there is an excess of bearing surface, increasing frictional forces. If the spring is rotated too quickly, then excessive heat buildup may be generated, which increases the coefficient of friction on the silicone surface. A rotation rate of $120 \mathrm{rpm}$ with 1.2 coils/cm allows the shuttle to traverse the entire system length in approximately 50 seconds. A stepper motor with position control is used to automate the process and will be discussed later. 


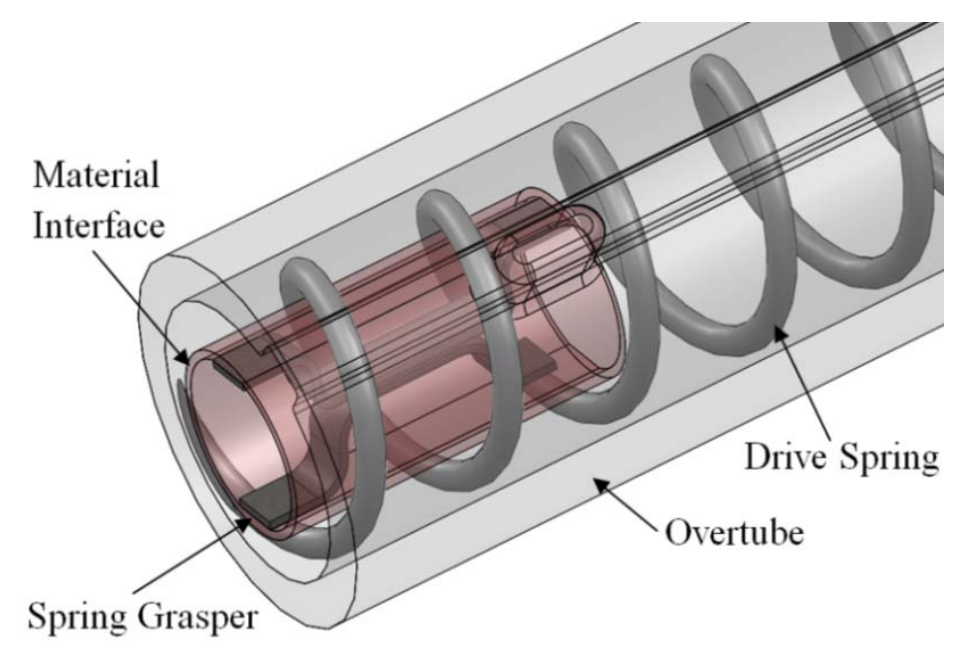

Figure 4-4: Primary functionality illustration

\subsection{Design Features}

In addition to the primary functionality of the system, several additional features add value to the overall system's impact on NOTES procedures. These additional features are steering, lighting and video capability, suction, and irrigation. To add these features, modifications to the overtube geometry in Figure 4-4 must be made. Adding secondary and tertiary lumens allows for these features to be added. Although most overtubes used in endoscopy are cylindrical in shape, the esophagus is relatively compliant, and will conform to quasi-round shapes with an effective diameter $\leq 25 \mathrm{~mm}$ [4-2]. Effective diameter is calculated by taking the total perimeter of the non-round cross-section and calculating the diameter of a round tube with the same perimeter. Placing three lumens into a non-round tubing cross-section will enable a more compact design when compared to a round OD with the same three lumens (Figure 4-5). 


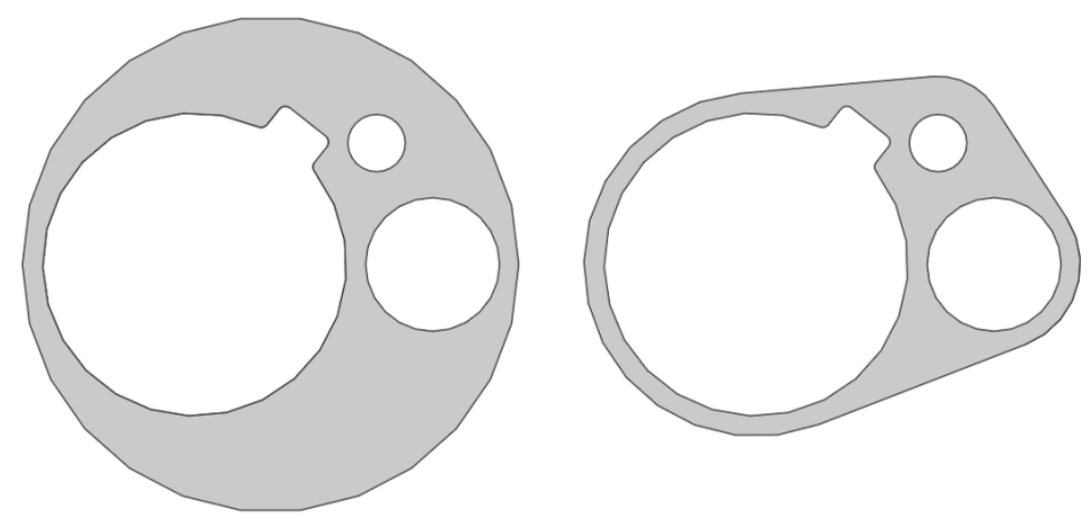

Figure 4-5: Comparison of round vs. non-round cross-sections

\subsubsection{Steering}

Having the ability to steer the system as it is inserted through the gastrointestinal tract and into the peritoneal cavity is paramount for proper placement. Steering the device can be achieved by a number of methods including custom shape memory alloys (SMAs), cables, hydraulics and pneumatics. Steering can also be achieved by inserting an endoscope or articulated fiberscope into the overtube. Because of the sensitivity of SMAs to minor temperature changes, the relative complexity of cable driven systems, and the relative bulk of pneumatic systems, inserting a pre-existing articulated device such as a flexible borescope (fiberscope) or endoscope into the system is the best option, shown in Figure 4-6. 


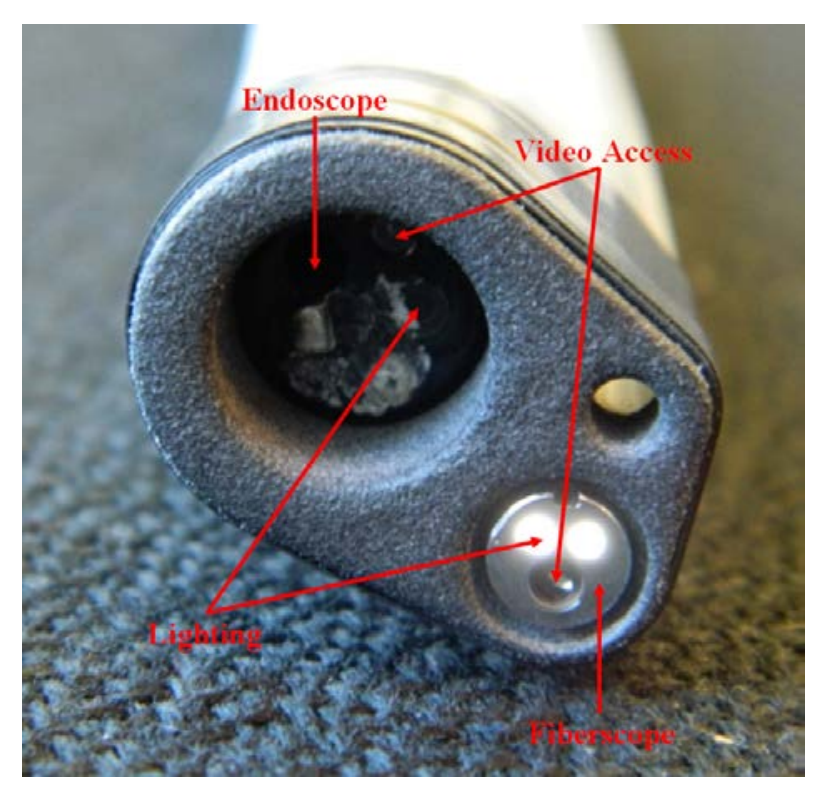

Figure 4-6: Standard endoscope and articulated fiberscope inserted into the overtube

The system has been designed to accommodate both standard 11mm endoscopes and standard $6 \mathrm{~mm}$ fiberscopes. The endoscope will fit in the main working channel of the MHS; however, the drive spring and shuttle must first be removed. These components have been designed in a modular fashion, meaning they can be removed and re-inserted as a monolithic assembly. Once the overtube assembly is in its desired location, the endoscope can be removed from the channel and the spring and shuttle assembly can be replaced. There is a secondary lumen in the overtube that will accept any flexible $6 \mathrm{~mm}$ inspection fiberscope. This channel is isolated from the rest of the working environment, eliminating the need for a medical-grade fiberscope. The primary advantage to using the fiberscope is that it can remain in the MHS for the duration of the procedure, enabling the use of its built-in lighting and video capability throughout a procedure, and eliminating the need to disassemble the main working channel of the device. 


\subsubsection{Lighting and Video}

As stated in the previous section, lighting and video capabilities can both be achieved through a secondary lumen by using a fiberscope.. A non-articulated fiberscope is significantly ( $\sim 10$ times) less expensive than the articulated variety. Most operating rooms that would be utilized for NOTES procedures would be equipped with an endoscope. Therefore a significant cost savings can be had by using an endoscope to articulate the tube, without investing in an articulated fiberscope. Having the nonarticulating fiberscope provides lighting and video capability throughout a procedure and adds little cost and minimal complexity to the device.

\subsubsection{Suction and Irrigation}

Suction and irrigation are commonly used during surgery [4-2], and as such, are useful additions to the Material Handling System. A 3mm channel with a $2 \mathrm{~mm}$ wall thickness is implemented to sustain the $-710 \mathrm{mmHg}$ pressure at which most surgical suction machines operate. To verify the capacity to withstand vacuum pressure, the overtube was attached to a roughing pump at one end, and sealed at the other. The tube was then bent to its minimum bend radius $(7.5 \mathrm{~cm})$, at which point no collapse was seen. Irrigation can also be passed through this same 3mm lumen.

\subsection{Final Design}

Figure 4-7 shows the modified system geometry with all of the additional aforementioned functionalities. The system, although having a maximum cross-sectional width of $28 \mathrm{~mm}$, has an effective circular diameter of $22.6 \mathrm{~mm}$, which is well under the allowable 25mm effective diameter of the human esophagus. 

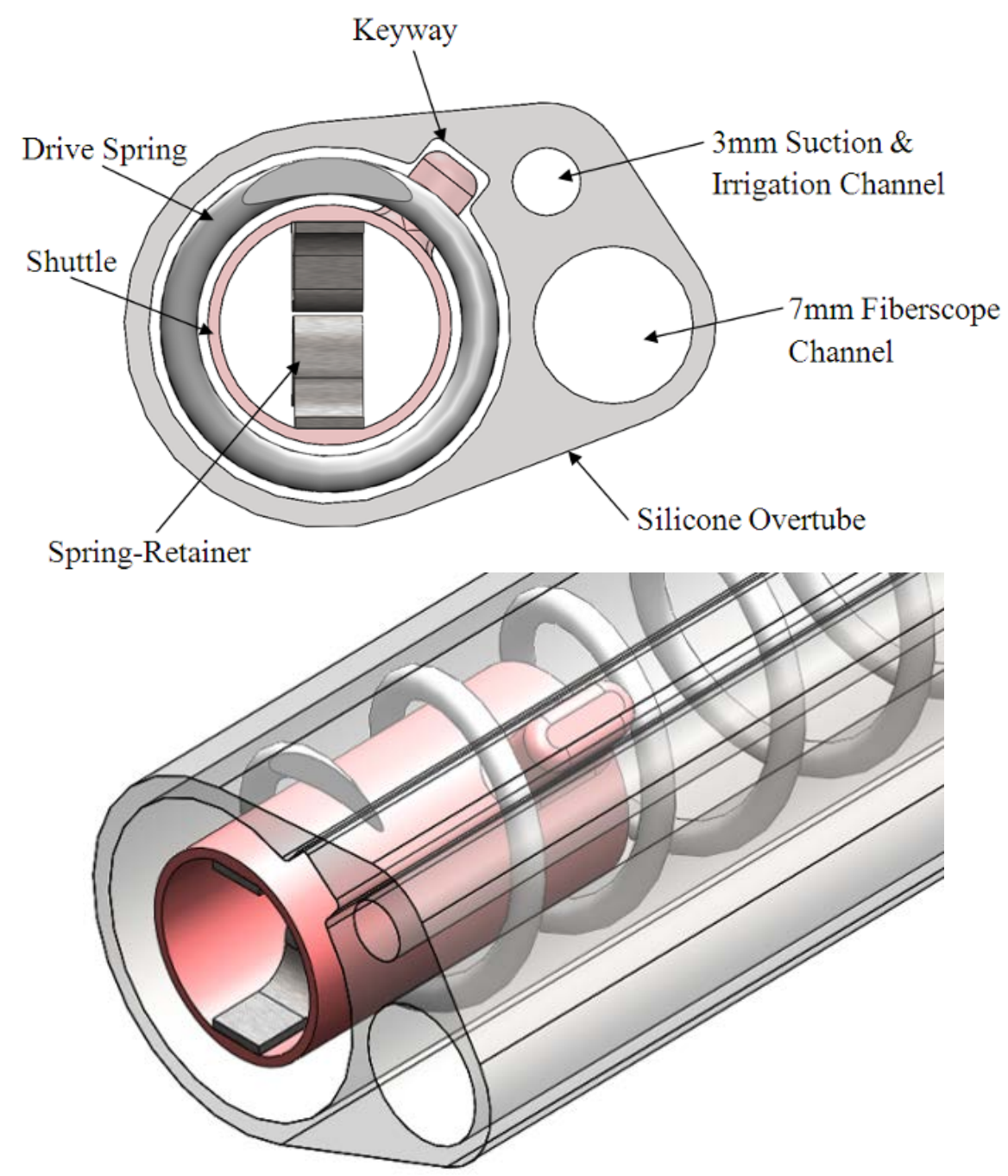

Figure 4-7: Material handling system cross-section

\subsection{Insertion Assembly - Other Details}

Payloads can be inserted or removed from either end of the system. The inserted end of the overtube has a simple retention cap to retain the spring and flexible endoscope (Figure 4-8). The distribution cap is more complicated as it routes all three lumens to their respective purposes; the primary lumen to the drive motor, the secondary lumen to a camera mount, and the tertiary lumen to a suction/irrigation port (Figure 4-9). 


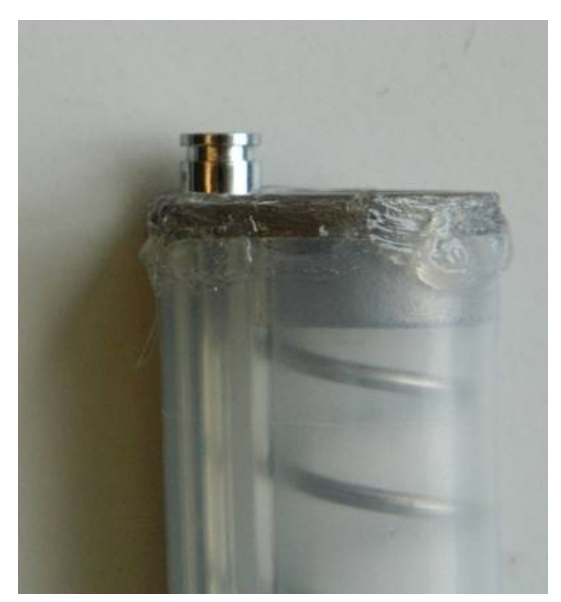

Figure 4-8: Inserted retention cap

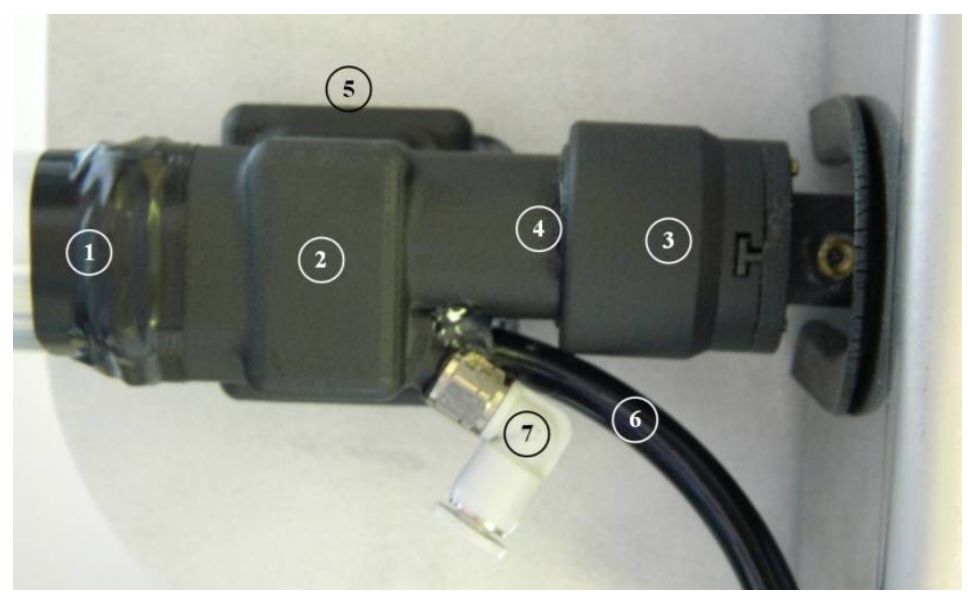

Figure 4-9: Distribution cap. (1) overtube, (2) grip pad, (3) motor coupling, (4) rotary insufflation seal, (5) magnetic attachment base, (6) fiberscope outlet, (7) 4mm quick connect suction/irrigation fitting

The tubing assembly is attached to a grip pad. The grip pad couples with the control interface via a neodymium magnet (Figure 4-10), and to the drive motor via a keyed coupling. The keyed coupling uses spring plungers to provide positive locking, while allowing for easy removal. The grip pad and keyed coupling have a hollow passageway that allows the surgical team access to the payload located within the shuttle, shown in Figure 4-11. 


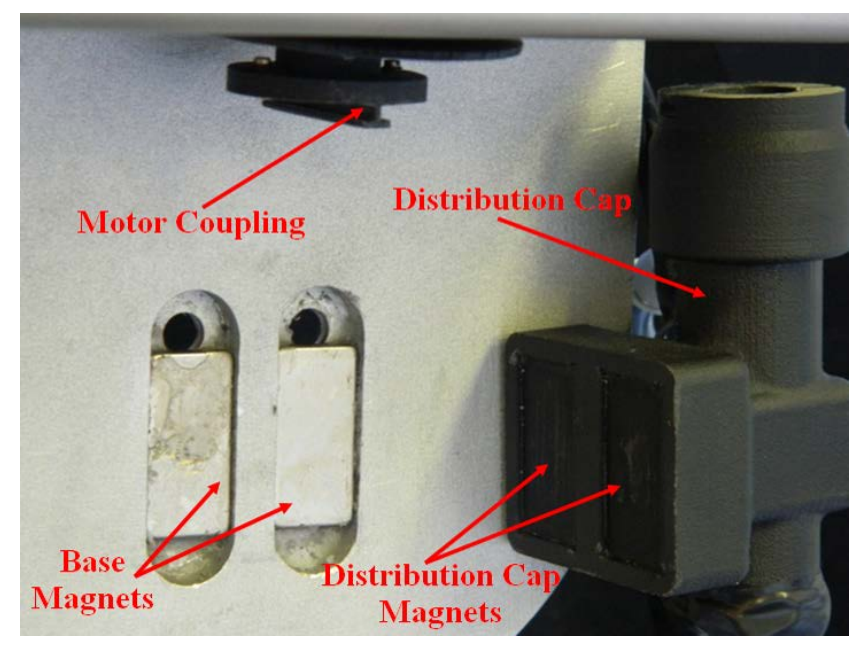

Figure 4-10: Magnetic attachment point for the distribution cap

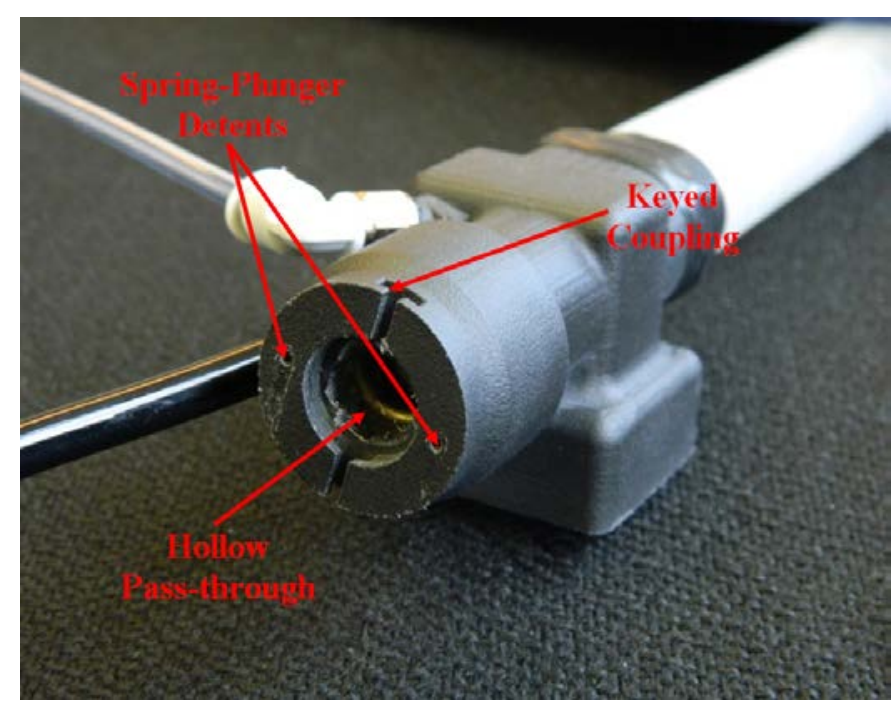

Figure 4-11: Grip pad and coupling diagram

One major concern during a NOTES procedure is maintaining abdominal insufflation. To maintain this pressure, there is a rotary insufflation seal (O-ring) between the grip pad of the distribution cap and the motor coupling. The distribution cap routes the fiberscope away from the coupling and into a camera, to allow for remote visualization. There is also a $4 \mathrm{~mm}$ flexible quick connector to hook up a suction/irrigation machine. When an operator wishes to insert or remove an object into 
the spring grasper, the distribution cap can be removed with one hand by grasping the grip pad and perpendicularly pulling the assembly away from the control interface.

\subsection{Special Manufacturing Considerations}

The design of the silicone overtube was largely dictated by manufacturability of the product. Extruding small features $(<1 \mathrm{~mm})$ is possible on tubing with lumens less than $10 \mathrm{~mm}$ in diameter. From $10-25 \mathrm{~mm}$, feature sizes no less than $1 \mathrm{~mm}$ are possible, as the material will not flow through the extrusion dies properly. It is for this reason that a minimum wall thickness of $1 \mathrm{~mm}$ was required. Also, to improve the tolerances of the tubing, a high durometer ( 80 Shore A) was selected so that the material would flow more precisely during the extrusion process. Additionally, silicone rubber, once extruded, must be vulcanized to lock in the shape. Before the silicone is vulcanized, droop due to gravity can occur, such as shown in Figure 4-12A. To remedy this issue, the overtube was extruded vertically, shown in Figure 4-12B.
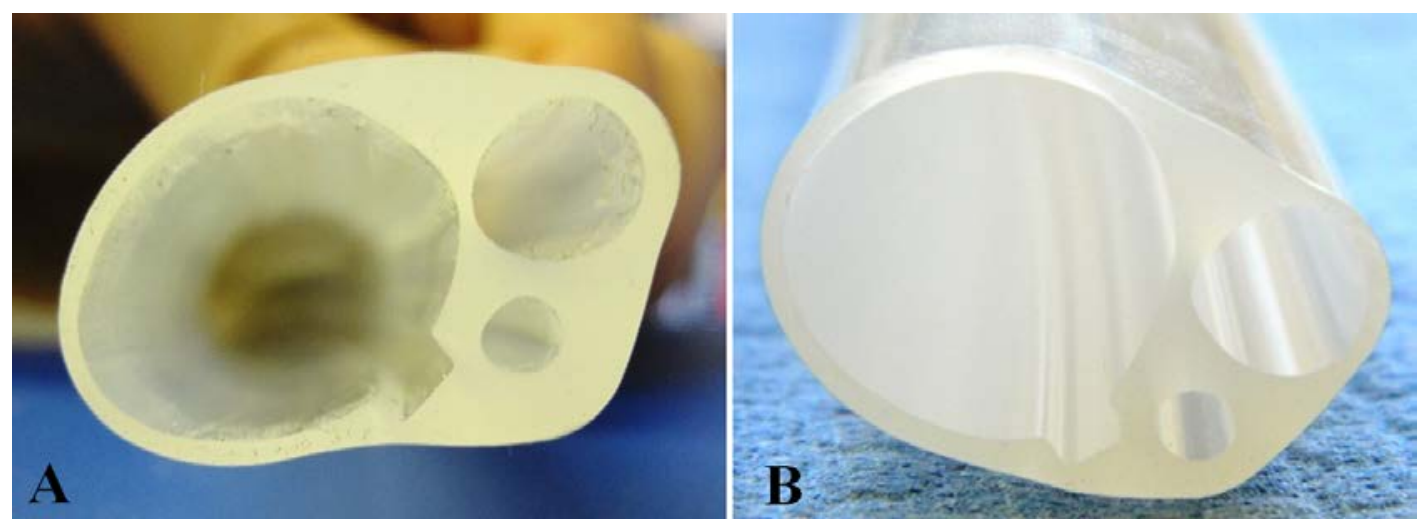

Figure 4-12: (A) Horizontal extrusion droop; (B) Accurate vertical extrusion 


\section{Chapter 5: Electronics and Control System}

\subsection{Microprocessor and Motor Driver}

It is desirable to keep all electronics outside the harsh environment of the human body. The first and most critical component that must be selected is the control module. There are a number of different solutions available; including pre-configured GUI systems like National Instruments cRIO and the dSPACE prototyping system. These solutions are very expensive, and have far more capabilities than are required for the Material Handling System. The least expensive route is to choose a basic microcontroller that utilizes basic digital/analog I/Os and a common high-level programming language such as C. Two boards were selected for testing, the Ruggeduino and the FEZ Panda II, shown in Figure 5-1.

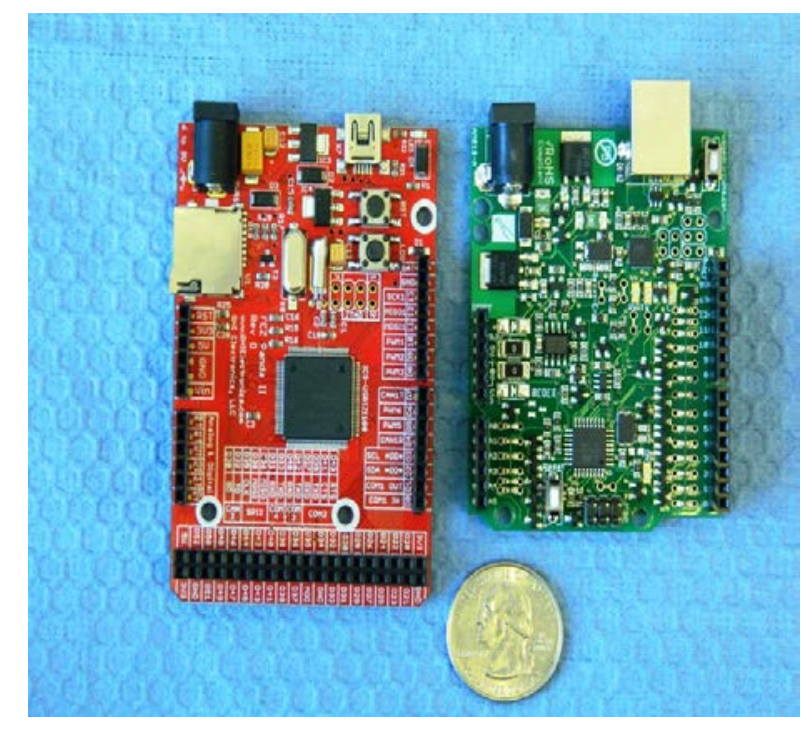

Figure 5-1: FEZ Panda II (left), Ruggeduino (right)

The Arduino platform is a very common hobby and prototyping microcontroller.

Programming is done in traditional C through an Arduino bootloader. The downside to the Arduino is that it only operates at $16 \mathrm{MHz}$, and has a limited number of I/Os. As an 
alternative, the 72MHz 32-bit FEZ Panda II is a so-called “netduino”, meaning it is based off the Arduino platform and can utilize all of the same shields and hardware, but is instead programmed from the .NET framework through Windows in C\#. Programming in .NET is more complex, and taking advantage of the 32-bit multi-threading capabilities proved difficult. Ultimately, the Ruggeduino, a more durable version of the Arduino, was chosen for its open-source architecture, programming simplicity, and the availability of pre-configured and coded motor shields. Furthermore, Hribernik et al. [5-1] concur that the Arduino platform is an excellent method for quickly and easily prototyping quite complex intelligent products.

The drive spring on the insertion assembly required very precise position control, and it was ideal to keep all of the sensors external to the body, so an open-loop control strategy was a reasonable logic configuration. For this control, a 200-step optical encoder was selected, in conjunction with a 200-step NEMA 17 high-torque stepper motor (Anaheim Automation), to operate the drive spring. A stepper motor is ideal for making incremental movements without requiring external sensors to monitor the rotation. The optical encoder was selected to act as a redundant measure for maintaining an accurate reading of the angular position. Matching the step count between the optical encoder and the stepper motor is important for the open-loop system control. To drive the stepper motor, the Adafruit motor shield was selected, shown in Figure 5-2. The shield is equipped with a dual H-bridge configuration, which is ideal for driving a number of different types of motors. Additionally, there were several open-source libraries in the Arduino programming GUI that allowed for easy programming of the shield. 


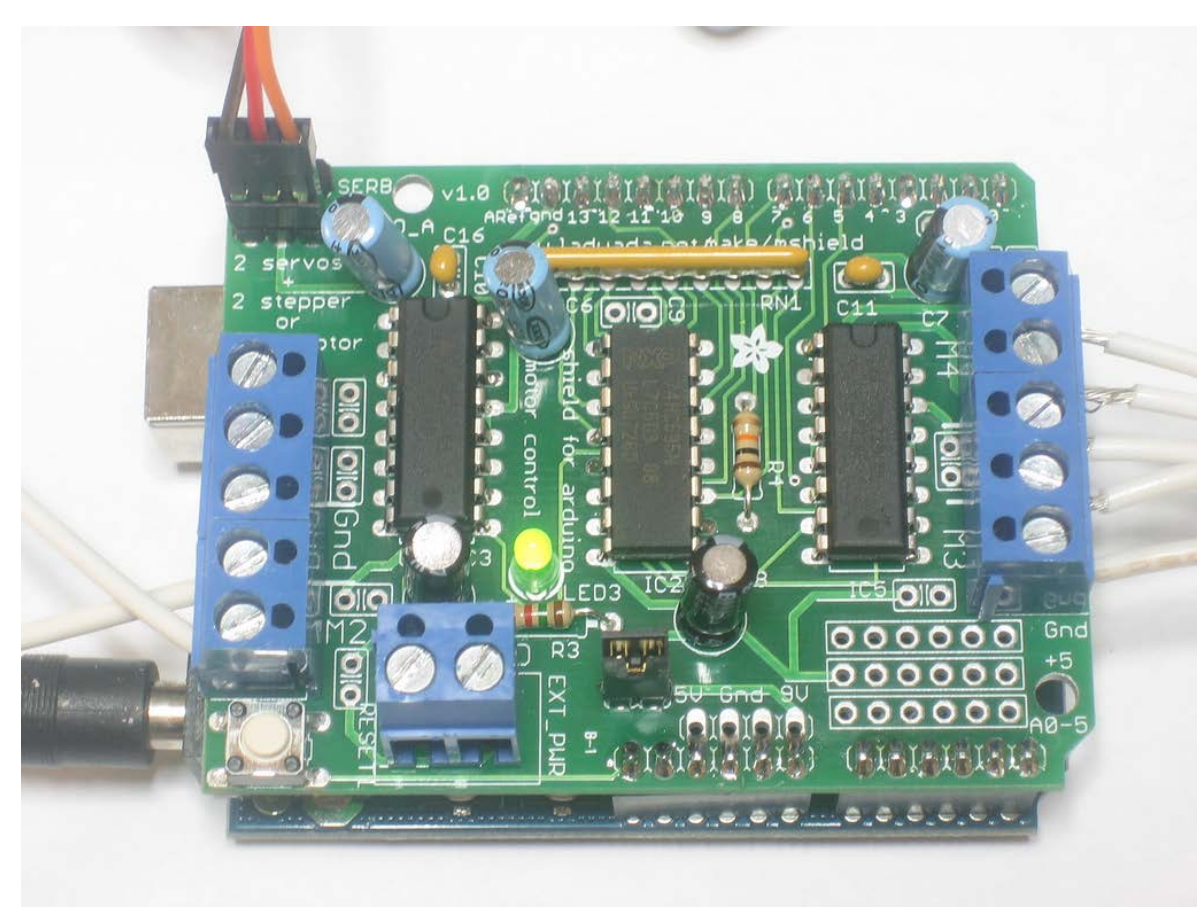

Figure 5-2: Adafruit motor shield

The stepper is single stepped, giving it a $1.8^{\circ}$ incremental step angle. This step angle coupled with the 1.2 coils/cm drive spring yields a small linear step size of 0.05 millimeters. This step size allows for fine linear placement of the material interface.

\subsection{Interface \& Open-Loop Control}

An open-loop control strategy is implemented for maintaining the simplicity and durability of the Material Handling System. The control strategy, shown in Figure 5-3, enables the system to be operated in either automatic or manual jog mode. 

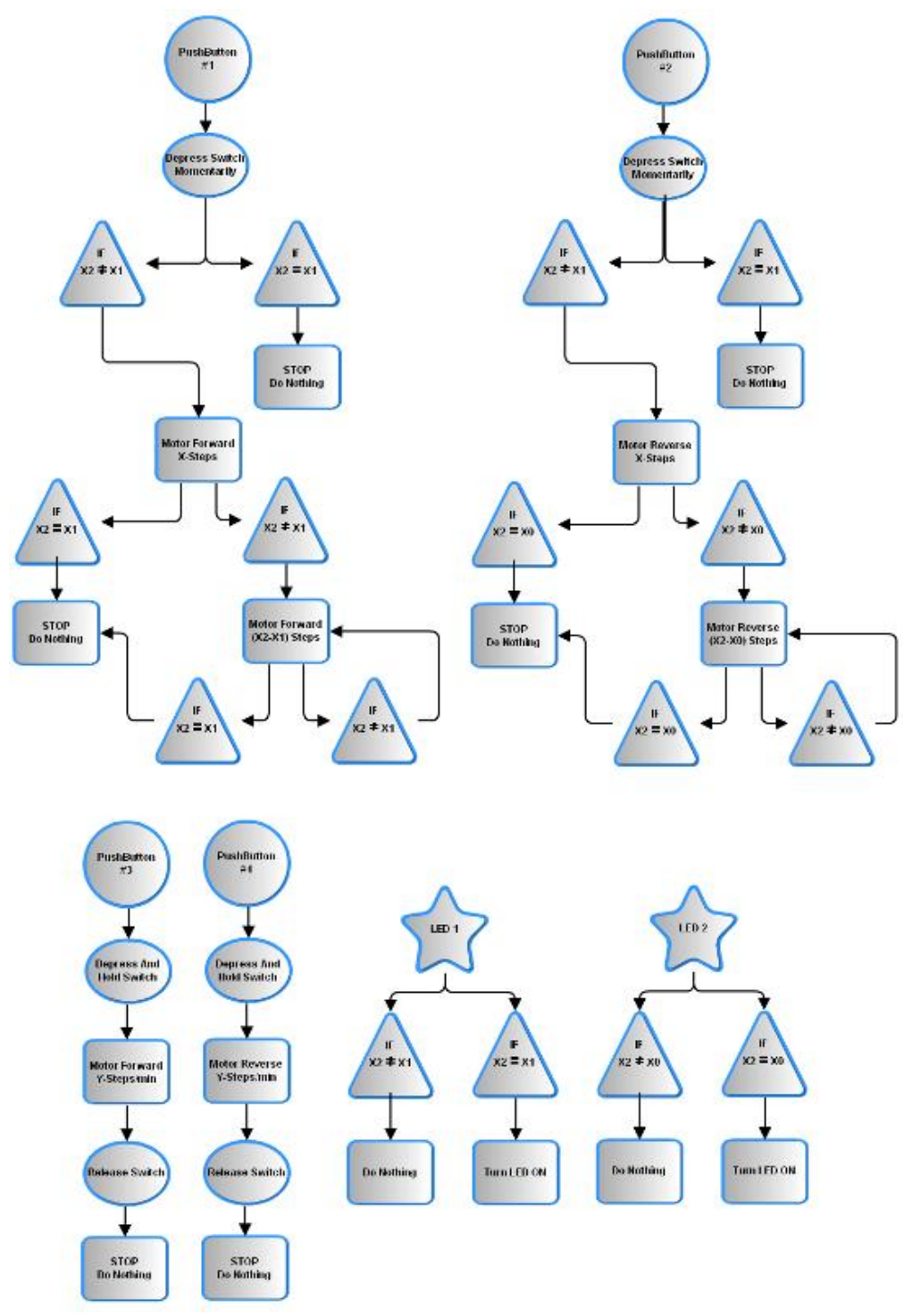

Figure 5-3: Open-loop control schematic

The control interface has four buttons, retract automatic, insert automatic, retract manual and insert manual. Both operational modes utilize LEDs to indicate whether the shuttle is fully inserted or fully retracted (Figure 5-4). 


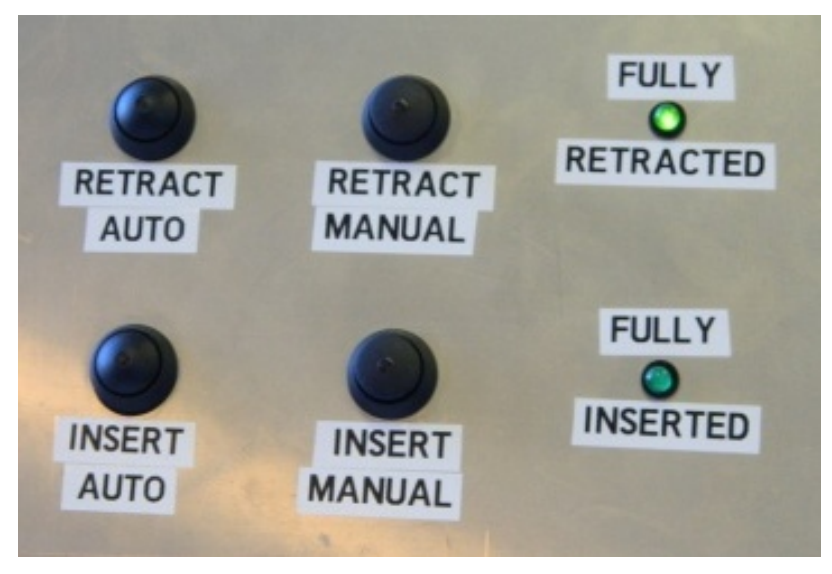

Figure 5-4: MHS control interface

When placed in automatic mode, the shuttle traverses the entire length of the overtube. The microcontroller sets the number of step impulses to the drive motor, corresponding to the overtube length, and monitors the position of the shuttle by counting lines from the optical encoder. If the number of motor pulses matches the encoder reading, the shuttle has traveled the appropriate distance. The main downside to a stepper motor is that if the maximum rated torque is exceeded, then slipping can occur, thus losing the index for true position. If the overtube is twisted or obstructed such that the motor slips, the encoder does not match the number of motor steps. The microcontroller automatically calculates the remaining travel distance and sends that number of step commands to the motor. The system also has soft stops built into the operation code to prevent the shuttle from crashing into either hard stop at the ends of the overtube. When either soft stop is reached, an indicator illuminates, alerting the operator of the shuttle position. If the shuttle were to reach a hard stop, the drive spring would bind and cause the motor to slip, but no component failure would occur.

Depressing the manual button moves the shuttle until the button is released, or until a soft stop is reached. The system can be placed in automatic mode even after being 
manually operated; the microcontroller automatically adjusts the number of automatic step commands based on the shuttle’s current position.

\subsection{Other Hardware}

There are a few other components included in the design of the control interface that are worthy of brief discussion. The system utilizes a 75W dual power supply. The Ruggeduino, Adafruit motor shield and stepper motor utilize a 24V supply, and the LEDs and momentary contact pushbuttons utilize a 5V supply. Once testing commenced, switch bounce, a common issue with momentary contact pushbuttons, was apparent. Essentially the contacts within the button do not maintain contact upon initial depression, and as a result, so-called phantom signals were causing the MHS to cycle unintentionally. To correct the issue, an RC circuit with an inverter, specifically designed as a switch debouncer, was implemented and is shown in Figure 5-5.

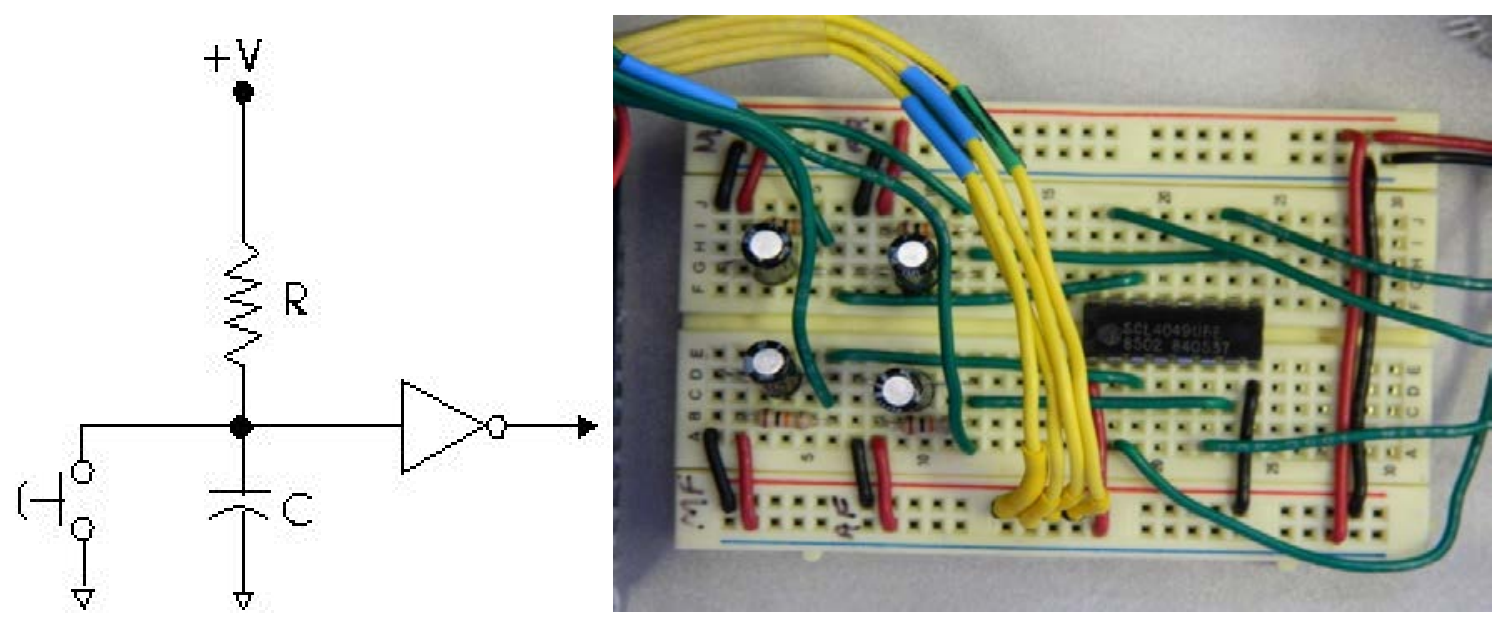

Figure 5-5: Debounce circuit diagram (left), implemented circuit (right) 
The other issue that developed during testing was heat build-up on the motor. The stepper motor was wired to use its maximum allowable power level to optimize torque levels. Additionally, when the MHS is idle, the motor is set in full-lock mode, which essentially sends full power to all of the motor poles. To remedy this heat, a large heat sink was attached to the motor, and two quiet, high velocity computer fans were also placed within the electronics panel. The fully assembled electronics package is shown in Figure 5-6.

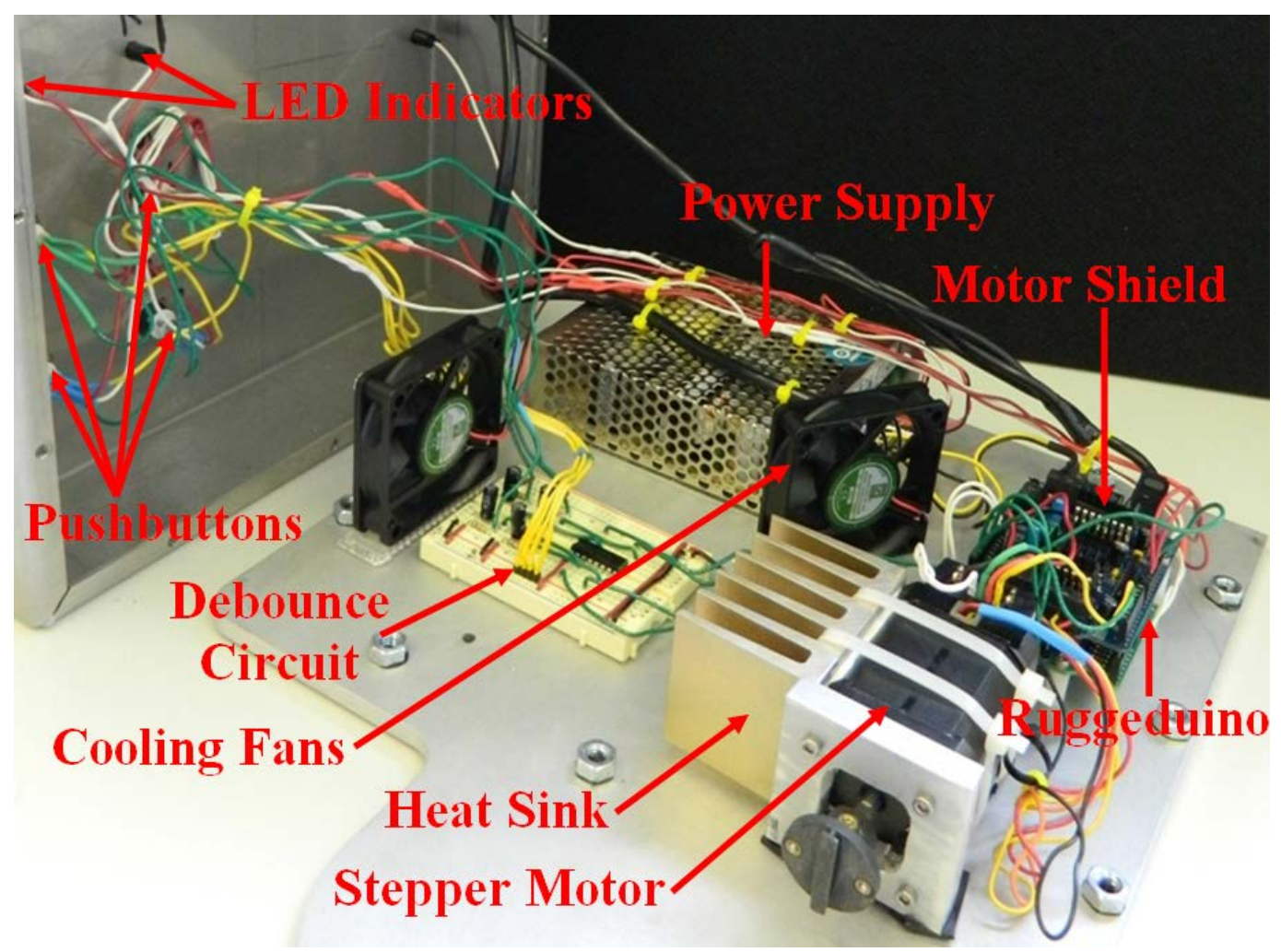

Figure 5-6: Completely wired electronics package 


\section{Chapter 6: Bench-Top Testing}

\subsection{Reliability}

The complete system has been subjected to a number of tests to quantify satisfaction of functional requirements. Primary success of all tests is based on visual verification that the shuttle can navigate the length of the overtube in both directions. The first test is a repeatability test, in which the system was subjected to a $12.7 \mathrm{~cm}$ bend radius and an axial twist of $180^{\circ}$ (Figure 6-1). These parameters were chosen as an approximation to the type of compound flexure that may be encountered during a transgastric insertion. The system was repeatedly cycled in automatic mode and visually inspected to verify successful movement and final placement of the shuttle in the overtube. The operation was also timed, and the position of the shuttle relative to the retention cap and to the insufflation cap was measured. Across 20 samples, the time of travel was $55.8 \pm 0.2$ seconds in both directions, yielding an average speed of 1.8 $\mathrm{cm} / \mathrm{second}$. The distance from both caps was measured with the heel of a dial caliper, and was within $1 \pm 0.15 \mathrm{~mm}$ across the sample set. 


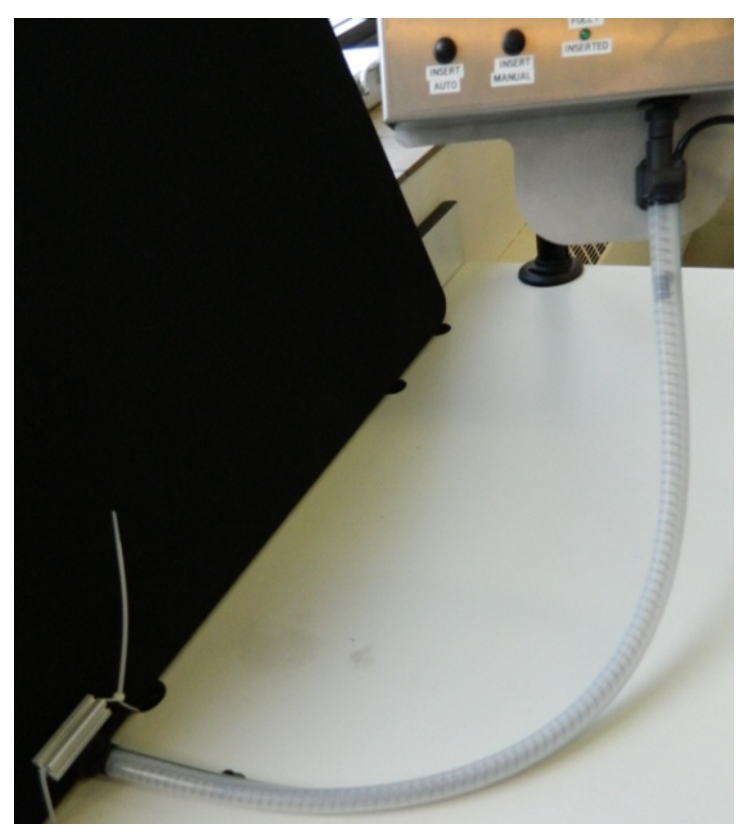

Figure 6-1: Repeatability test: $12.7 \mathrm{~cm}$ bend radius, $180^{\circ}$ axial twist test

\subsection{Maxima: Bend Radius \& Axial Twist}

The next tests performed included finding the maximum allowable bend radius and axial twist of the system, without the drive spring binding, which would cause the stepper motor to slip. Allowable bend radius was variable depending on the direction of bend, as the wall thickness of the overtube is not uniform in all directions relative to the material interface lumen. The bend radius was applied manually at the midpoint of the overtube length. A radius of $7.5 \pm 0.63 \mathrm{~cm}$ was attainable in the upward, downward and left directions, and a radius of $12.7 \pm 0.63 \mathrm{~cm}$ was attainable in the right direction (Figure 6-2). 


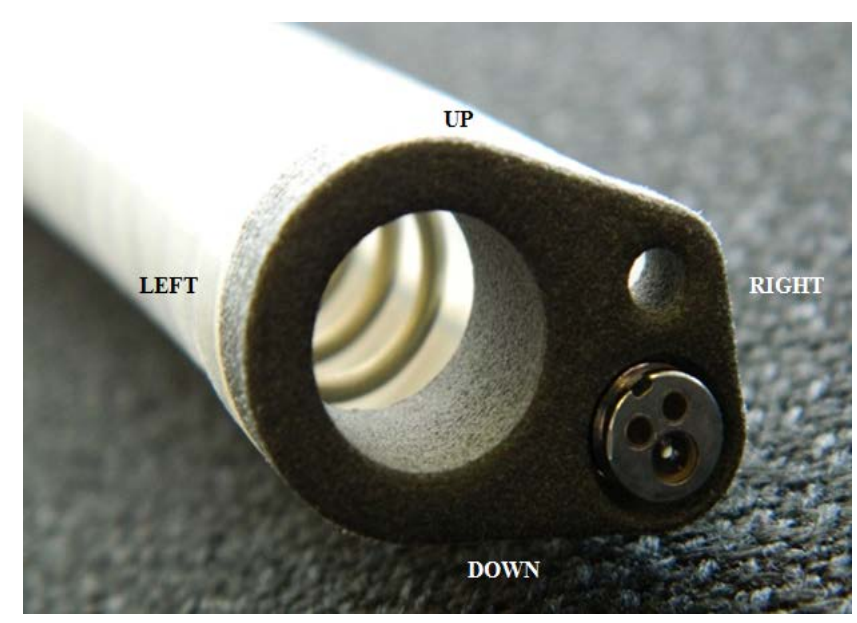

Figure 6-2: Bend radius orientations

To obtain the maximum axial twist of the insertion assembly, the distribution cap was attached to the magnetic mounting pad normally, and then the inserted retention cap was rotated. An axial twist over the entire length of the overtube exceeding $360^{\circ}$ allowed for successful system operation. Greater axial twist values may have been acceptable, but the torque required to displace the tube beyond that value caused the magnetic attachment to become uncoupled, rendering the tests infeasible.

When the system was pushed beyond its maximum limits, the spring did bind, and caused the motor to slip. Once the system was placed back within its operating bounds, the microcontroller was still able to adjust the travel distance appropriately and deliver the payload to the appropriate soft stop with the same accuracy found during the repeatability test. 


\section{Chapter 7: Porcine Testing}

\subsection{First Procedure}

As part of the functional verification of the MHS, in vivo testing on a non-survival porcine model was performed. The goal of the procedure was to insert the device transgastrically and operate the system as designed, thus validating device functionality in a harsh environment. The anatomy of a pig esophagus is prohibitively small at the entry point, both in diameter and bend radius. To avoid this restriction, an incision was made in the neck, just below the natural bend of the esophagus, shown in Figure 7-1. During the attempt to perform insertion of the device, some excessive tearing of the tissue occurred, making the transgastric insertion impossible. This tearing can be attributed to the smaller overall diameter of the pig esophagus compared to the average human esophagus. As the MHS is designed for the anatomy of a human, this outcome, although not desirable, is acceptable.

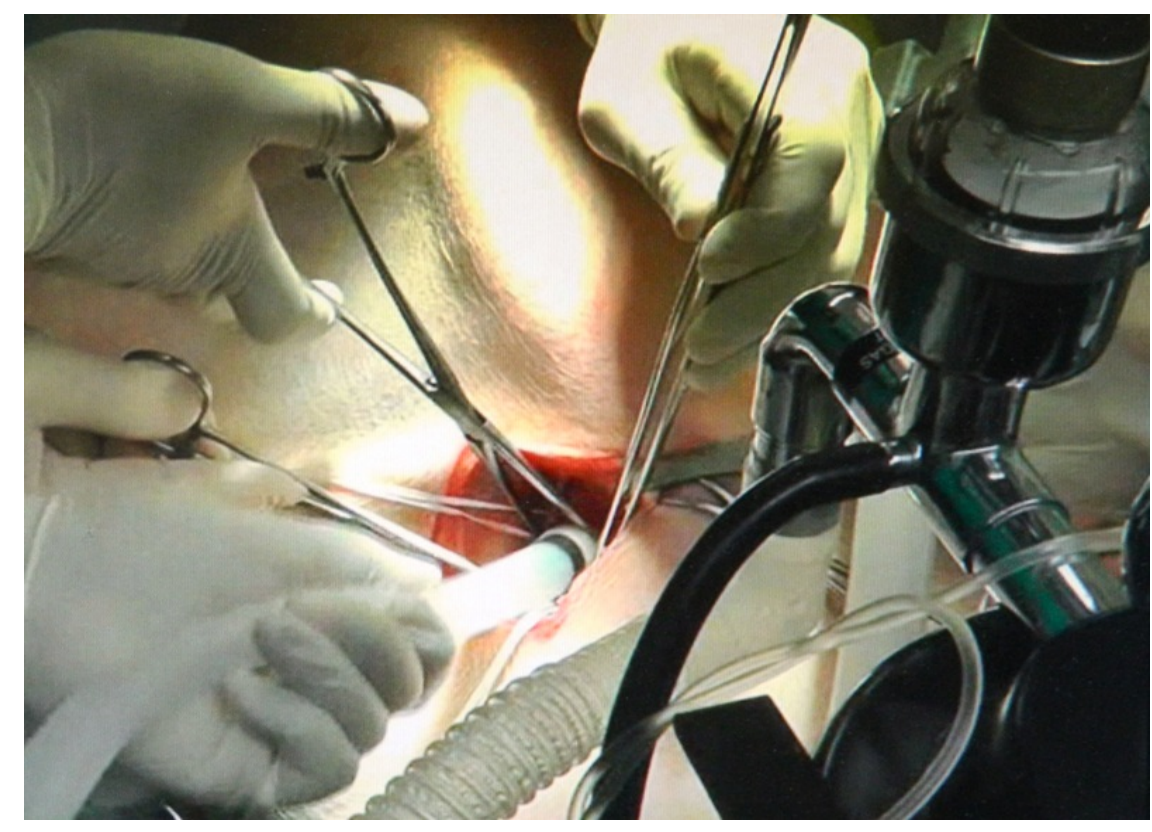

Figure 7-1: Transgastric insertion attempt 
As an alternative to the natural orifice insertion, the MHS can be inserted through an external incision in the abdominal cavity. The porcine model was insufflated with two $12 \mathrm{~mm}$ trocars, and an additional $50 \mathrm{~mm}$ gel port was inserted at the abdominal midline of the animal. After the unsuccessful attempt to enter transgastrically, the system was instead inserted into the insufflated peritoneal cavity via the gel port. Approximately $45 \%$ of the 1 meter long overtube assembly was introduced to the harsh environment of the abdomen (Figure 7-2).

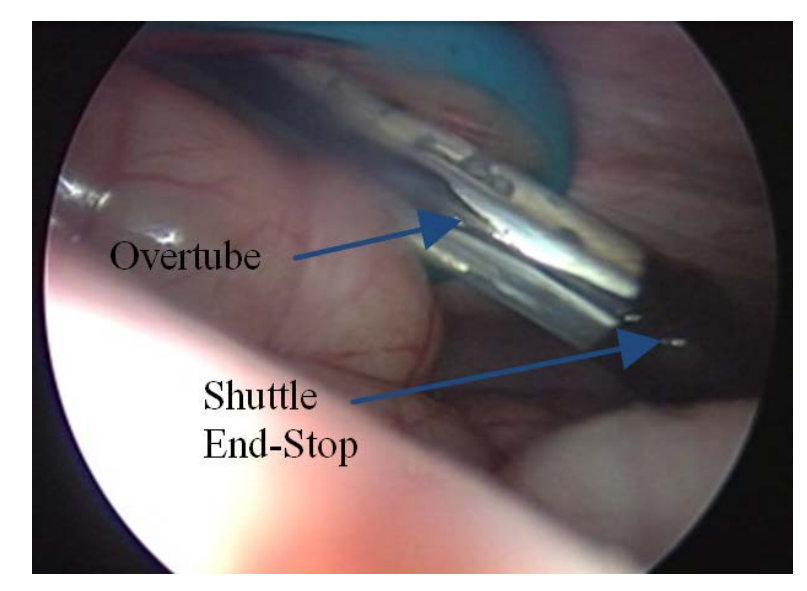

Figure 7-2: System inserted in vivo via gel port

Once inserted, the system was operated in automatic mode, cycling the system multiple times from fully retracted to fully inserted. Additionally, a surgical staple was placed within the spring grasper while the shuttle traversed the overtube, and was held securely. Both with and without a payload, the MHS cycled properly, even under some unusual bend angles induced by the gel port location. It was determined by visual inspection that the shuttle was delivered to the appropriate soft stops after each cycle. These results confirm that even in the wet and sticky abdominal cavity, the Material Handling System is a robust device. 


\subsection{Improvements \& Second Procedure}

\subsubsection{Insertion Trocar}

The first procedure elucidated two deficiencies in the MHS: the lack of a proper insufflation seal, and the lack of a robust way of gaining natural orifice access in a porcine model. Although the system is designed for a human, all medical devices must be tested and validated in vivo before gaining approval for human testing. An oversized trocar was designed to allow natural orifice insertion via a transvaginal approach. The trocar is designed to mimic other production trocars, such as the ones described by Fuller et al. [7-1], and shown in Figure 7-3.

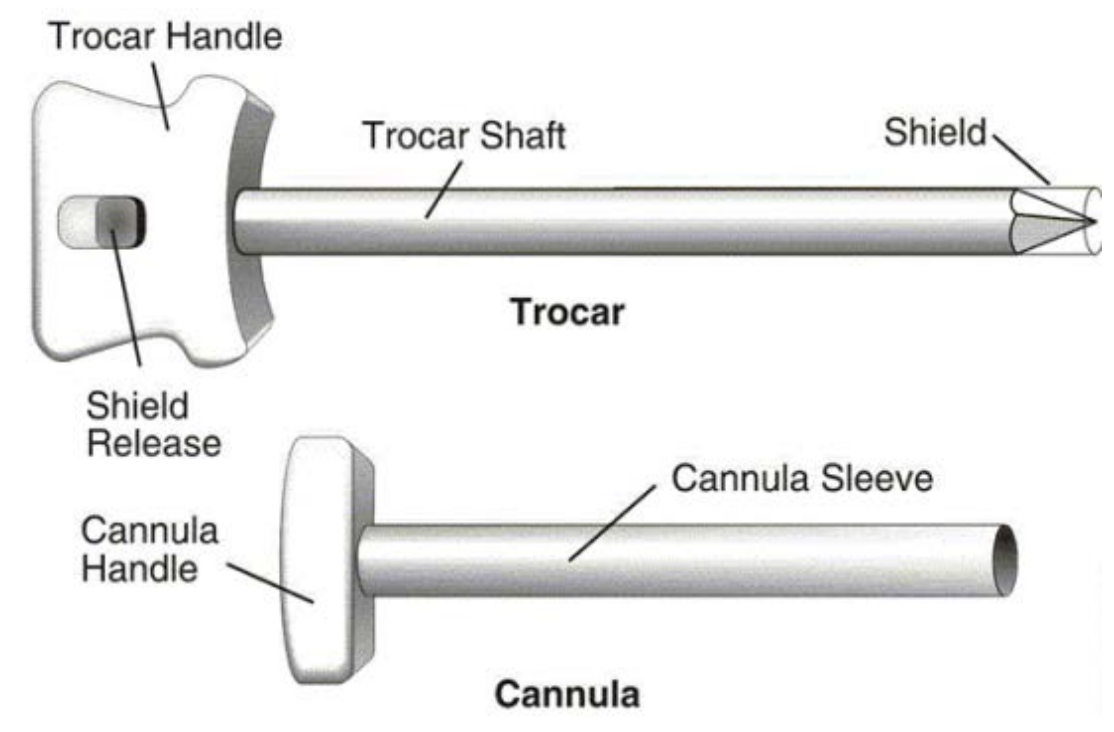

Figure 7-3: A typical trocar configuration, listed by the FDA [7-1]

The MHS trocar is composed of a 1.125 inch $(28.6 \mathrm{~mm})$ diameter cannula sleeve, through which the piercing trocar passes. The entire retracted assembly passes transvaginally and when located appropriately near the interior abdominal wall, the piercing tip can be extended, puncturing an appropriate size hole such that the cannula 
sleeve can be advanced. The retracted and extended trocar configurations can be seen in Figure 7-4.

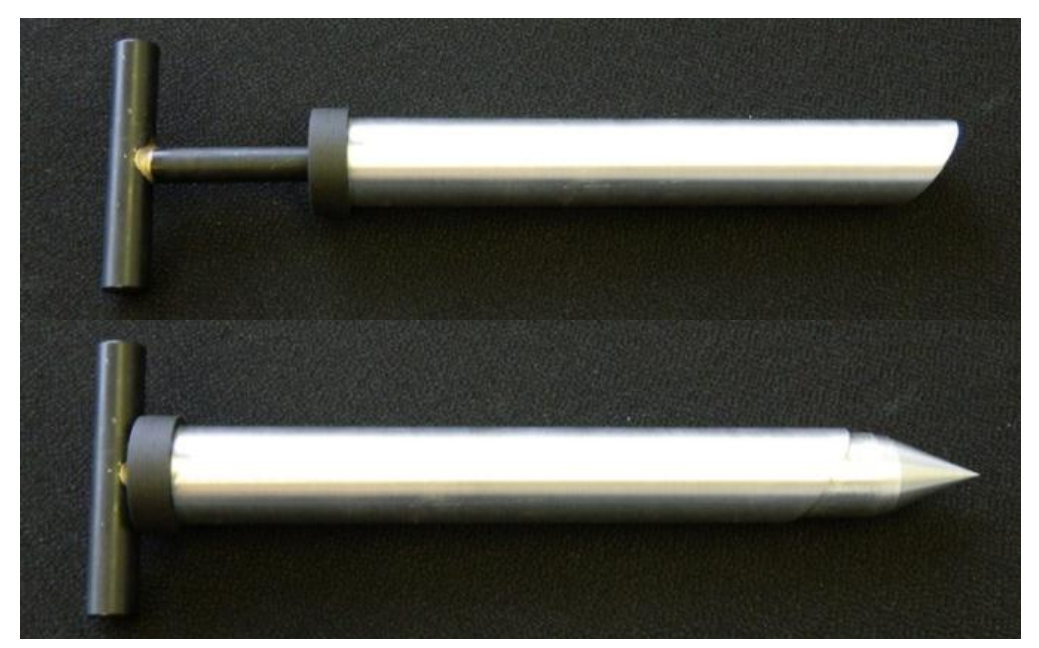

Figure 7-4: MHS trocar in the retracted (top) and extended (bottom) configurations

The trocar is equipped with three O-ring insufflation seals to maintain abdominal insufflation during puncture. Once inserted, the piercing trocar is removed from the cannula sleeve, and the overtube insufflation cap is placed over the end of the sleeve. Once lubricated, the MHS overtube can be advanced through the cap and the cannula sleeve into the abdominal cavity. The tight fit and lubrication between the cap and overtube will maintain insufflation within the abdomen. A diagram of all critical components can be seen in Figure 7-5. 


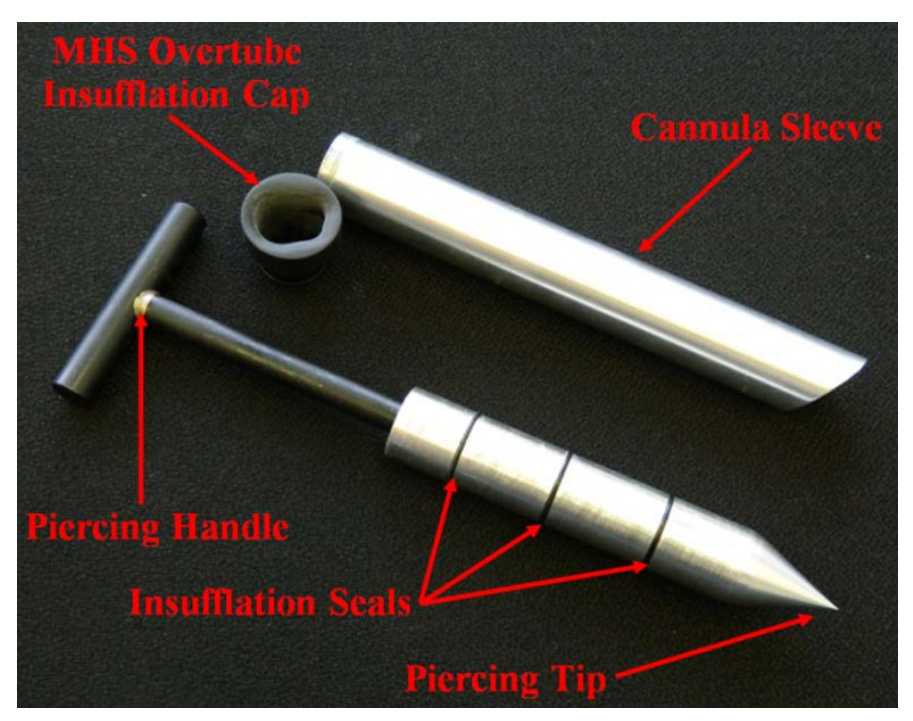

Figure 7-5: MHS trocar components

\subsubsection{Second Procedure}

With the addition of the trocar, natural orifice insertion proved to be a rather simple approach. During the second procedure, the trocar was inserted transvaginally, with the piercing tip initially retracted. Once the trocar was fully inserted into the vagina, the piercing handle was advanced, and the tip passed easily through the vaginal wall and into the insufflated abdominal cavity, shown in Figure 7-6. At this point, insufflation was fully maintained without any noticeable loss in pressure. The piercing assembly was then removed from the cannula sleeve, shown in Figure 7-7, at which point insufflation was lost. 


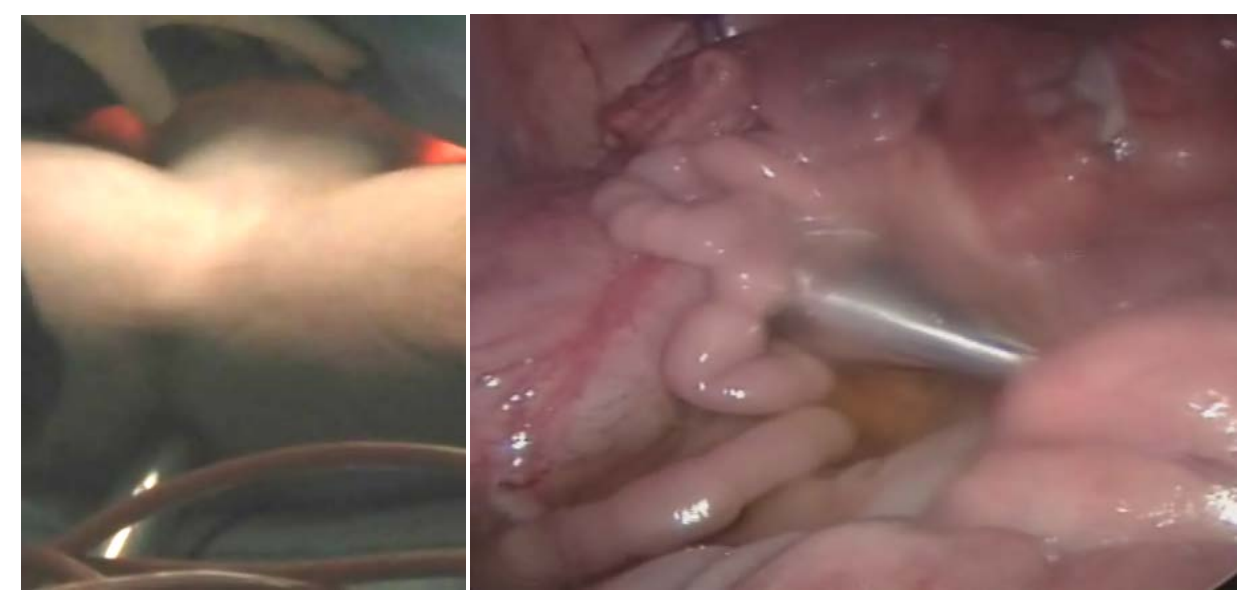

Figure 7-6: Transvaginal trocar insertion: exterior (left), interior (right)

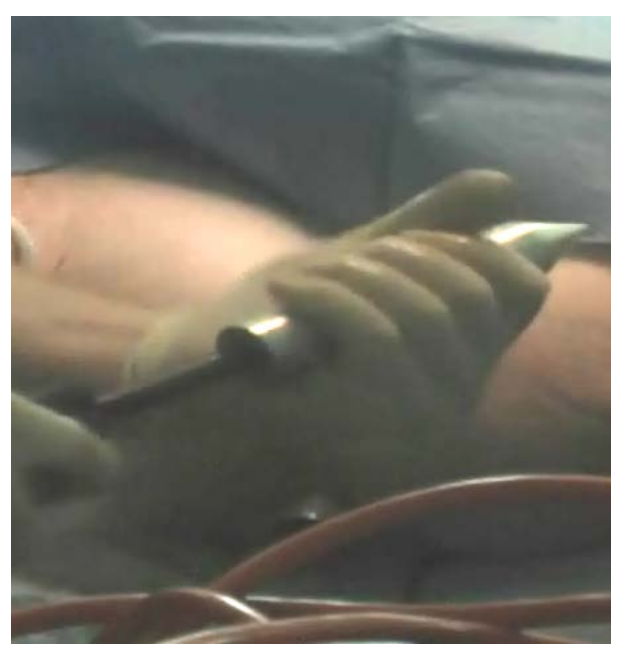

Figure 7-7: Trocar piercing assembly removed from cannula

The MHS was then inserted into the insufflation cap on the cannula sleeve and passed freely into the abdominal cavity, as shown in Figure 7-8. At this point, all of the insufflation seals, both at the trocar cap and throughout the MHS insertion assembly, worked as designed. A lifted abdominal wall is a clear indication of a proper insufflation seal, which is shown in Figure 7-9. 


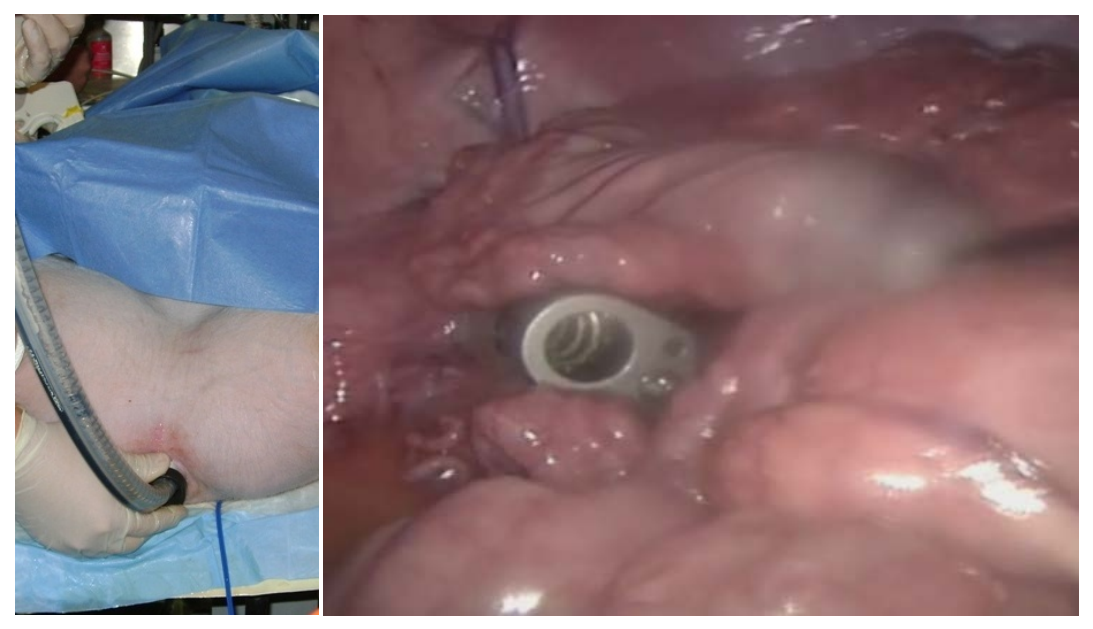

Figure 7-8: Insertion of MHS into trocar cannula: exterior (left), interior (right)

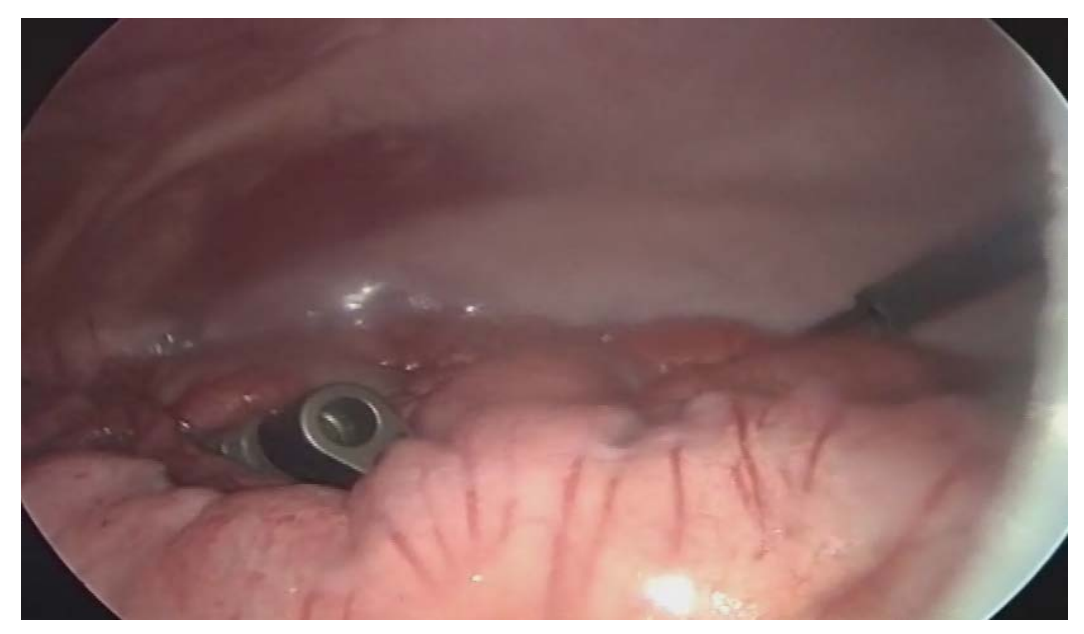

Figure 7-9: Raised abdominal wall with inserted MHS, indicating proper insufflation

The system was then actuated in automatic mode, advancing the shuttle through the trocar and into the abdominal cavity. As with the previous procedure, proper operation of the device is indicated with the delivery of the shuttle to the end of the MHS overtube. The shuttle was dyed blue for this procedure for clear indication, and can be seen in Figure 7-10. 


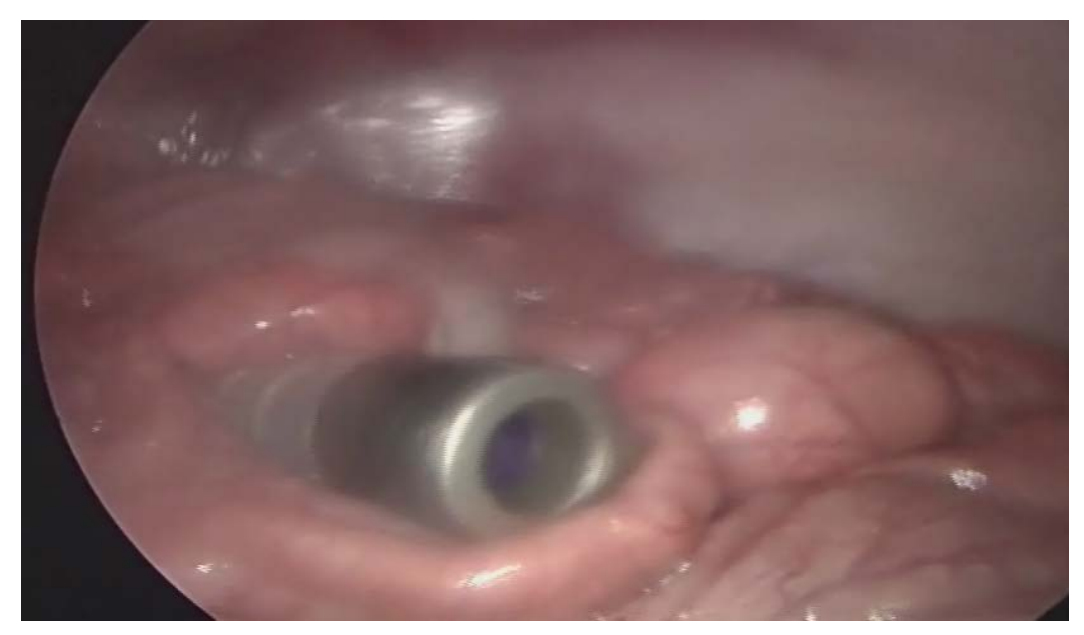

Figure 7-10: Fully inserted shuttle, dyed blue for proper visualization

The system functioned flawlessly as intended, both in automatic insert, and automatic retract modes, delivering the shuttle to the appropriate soft stops in each direction. This result was particularly impressive, as the bend of the tubing at the entry point of the trocar induced a large bend curvature, even causing some local ripple of the tubing. The robustness of the system was also confirmed as during the procedure, the bladder was inadvertently punctured, causing a large amount of urine to flow in and around the MHS. Regardless of this liquid, the entire system functioned properly. This procedure serves as a solid demonstration that the MHS is a reliable, robust, accurate system that can function during a multitude of abdominal procedures, and can be successfully inserted through a natural orifice. 


\section{Chapter 8: FE Analysis of Silicone Tubing Under Bending}

The silicone overtube comprises a large and very important portion of the design of the Material Handling System. As a continuation of the Material Handling System study, more investigation into the behavior of silicone tubing was desired. Determining the behavior of a silicone tube under bending can further improve the knowledge base for designing proper clearances to allow for correct operation of medical devices which make use of overtubes.

\subsection{Material Testing}

Structural silicone, specifically the medical grade NuSil MED 4080 silicone used in the Material Handling System overtube, can be a very difficult material to model. Any hyper-elastic materials with a high Poisson's ratio, such as many rubbers, have a complex response to loading, as a great deal of thickening/thinning can occur in the elastic range. Before any modeling could take place, material testing needed to be performed to ensure properties used in the simulation were accurate.

The FE material model selected and discussed in the following section requires the shear modulus, density and Poisson's ratio. The density and Poisson's ratio were provided by the manufacturer as $1210 \mathrm{~kg} / \mathrm{m}^{3}$ and 0.463 , respectively. The shear modulus was not published data, and therefore needed be determined experimentally. Shear modulus can be determined in a number of ways, but two different methods, tensile testing and ultrasonic testing, were utilized for this analysis.

Tensile testing was performed using a BOSE ElectroForce ${ }^{\circledR} 3200$ uniaxial test instrument with a 225N load cell (Figure 8-1). The system was calibrated, unloaded, using a built-in feature which varies the amplitude and frequency of a sine wave pulse. 
Once calibrated, three $45 \mathrm{~mm}$ long, $13 \mathrm{~mm}$ wide samples were loaded into the gripping jaws and stretched at a linear rate of $1 \mathrm{~mm} / \mathrm{second}$ to $6.5 \mathrm{~mm}$, a strain of $14.4 \%$. It should be noted that there was some minor hysteresis exhibited by the silicone as it was relaxed back to its initial length; however, that hysteresis is not applied to the FE material model.

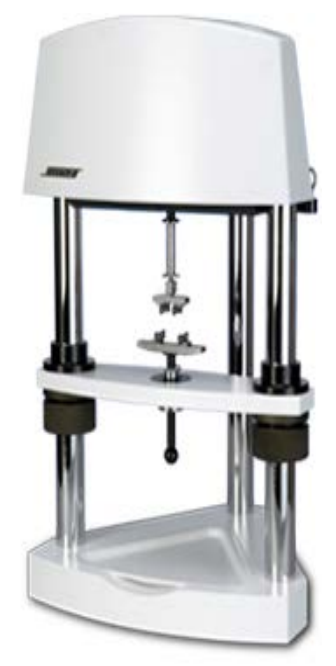

Figure 8-1: Bose 3200 ElectroForce ${ }^{\circledR}$ test instrument [8-1]

To determine the shear modulus of the material from the load-displacement data provided by the Bose machine, Young's modulus must first be found; this is the slope of the line fit to the engineering stress/strain curve (Figure 8-2).

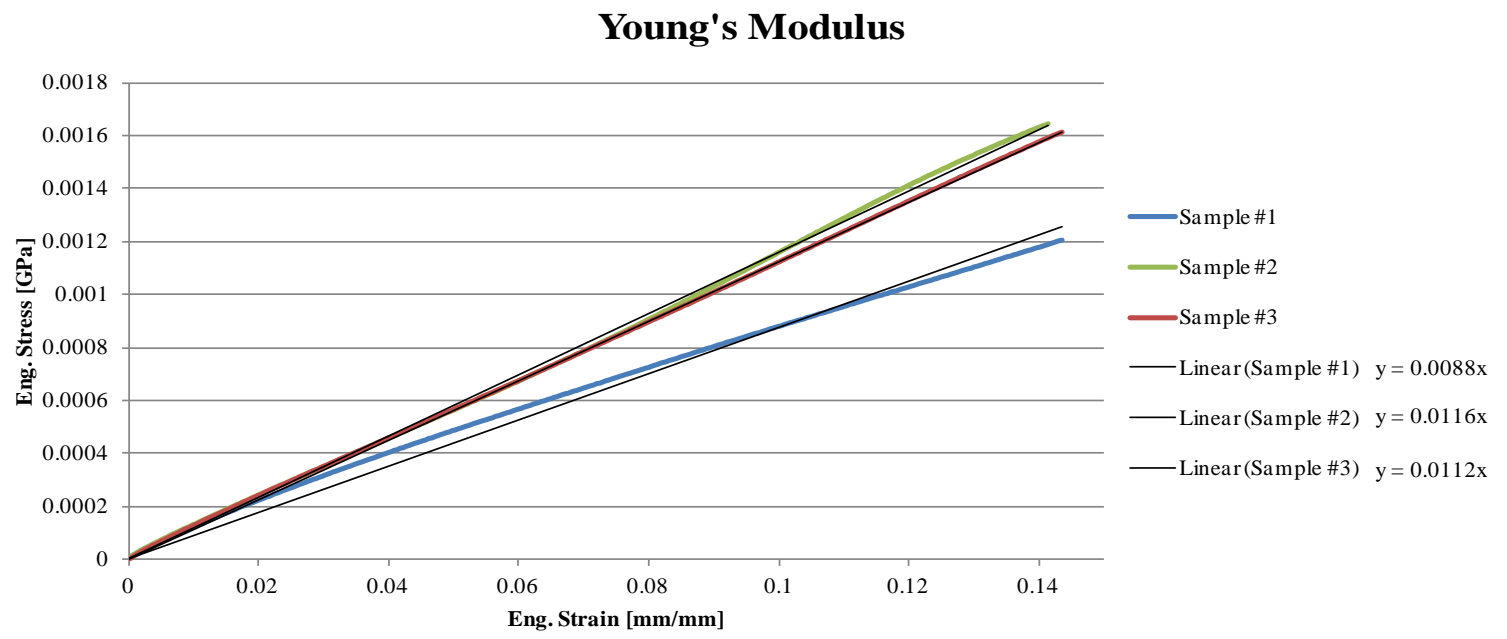

Figure 8-2: Young's modulus: engineering stress vs. strain 
An average Young's modulus of 10.5MPa was found. Knowing the Poisson's ratio $(\mu)$ and the elastic modulus $(E)$, the shear modulus $(\mathrm{G})$ can then be found (Eq. 8-1) [8-2]. An average shear modulus of 3.6MPa was found. According to published literature, this value is reasonably similar to previously tested structural silicones [8-3].

$$
G=\frac{E}{2(1+\mu)}
$$

As the shear modulus is not being directly measured, a secondary method of approximating the value is appropriate for validation. Ultrasonic testing is a widely accepted method for determining the elastic and shear moduli of materials [8-4]. All materials have an associated sound speed in both the longitudinal and shear directions which are directly related to the elastic and shear moduli, respectively. A common issue for materials with a very high Poisson's ratio, such as structural silicones, is that the sound speed of a shear wave is difficult to ascertain.

For the purpose of testing the MED 4080 silicone, a $5 \mathrm{MHz}$ longitudinal acoustic transducer was used to measure the sound speed. A Gaussian pulse is transmitted through the material, and then reflected back to the transducer. The initial waveform and two reflected waves were observed on a high resolution oscilloscope (Figure 8-3).

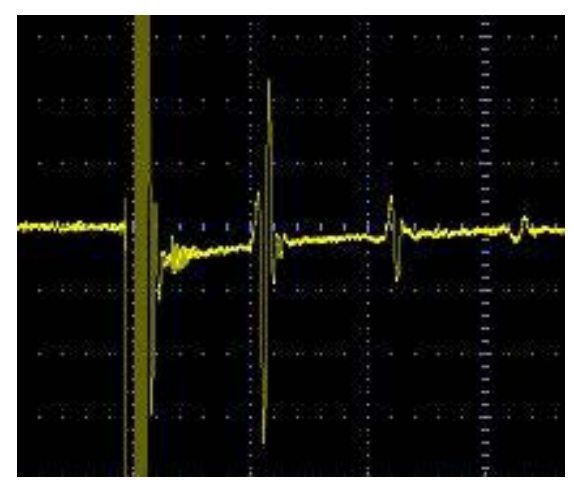

Figure 8-3: Longitudinal wave transmission and reflections ( $2 \mu$ s divisions) 
The sound speed of the material was obtained by measuring the time between peaks of the reflected waveforms. The travel distance of the wave (twice the material thickness) was divided by this peak-to-peak time, and resulted in an average longitudinal wave speed of $1164 \mathrm{~m} / \mathrm{s}$. Folds [5] published sound speed values of $960-1110 \mathrm{~m} / \mathrm{s}$ for RTV silicones at $0^{\circ} \mathrm{C}$. Using thermal correlation coefficients, at room temperature $\left(21^{\circ} \mathrm{C}\right)$, the expected sound speed would range from $1023-1173 \mathrm{~m} / \mathrm{s}$. Based on this literature, the longitudinal wave speed of this structural silicone is accurate. The correlation between longitudinal and shear wave speed is relatively straightforward (Eq. 8.2-8.4). Sample calculations are carried out in the Appendix. The shear wave speed of the silicone rubber is $305 \mathrm{~m} / \mathrm{s}$, resulting in a shear modulus of $113 \mathrm{MPa}$. This result is contrary to the previous tensile testing results and contrary to published data [8-3]. This unexpected result may be due to the high level of internal damping in the silicone. When testing the shear wave speed of a material such as steel, the sample is usually backed with a silicone rubber sheet, which is used to damp out any undesirable noise, or secondary reflections. This high damping may have caused the primary wave reflection to damp out or slow down on its way back to the transducer. This delay in transmission could be the error seen in the shear modulus.

$$
\begin{aligned}
& C_{\mathbb{L}}^{2}=\frac{\lambda+2 G}{\rho} \\
& \mu=\frac{\lambda}{2(\lambda+G)} \\
& C_{S}^{2}=\frac{G}{\rho}
\end{aligned}
$$


Despite that the ultrasonic sound speed tests seem to have provided an inaccurate result, FE simulation can go on with confidence, as the tensile testing resulted in comparable values to those published in literature.

\subsection{Material Model Verification}

The use of computer simulation to model the behavior of silicone is a difficult problem, and has been addressed in some detail previously [8-6]. A finite element engine, LS-Dyna, was utilized for the analysis of the MED 4080 silicone rubber. The previous literature suggests that using a one-parameter rubber model, namely the BlatzKo model, provides good physical correlation of the structural silicone, especially when strain levels exceed 20\%. The Blatz-Ko material model assumes that the Poisson's ratio of the material being analyzed is 0.463 and is intended for modeling nearly incompressible continuum rubber [8-7]. The parameters that need to be specified are the mass density and the shear modulus, both of which are now known.

To verify that the simulation is performing as intended, a single element pull test study has been conducted. In this study, and single shell element is loaded uni-axially, just like the tensile tests conducted in the previous section. The Blatz-Ko material model was used, with the default Belytschko-Tsay shell element formulation. The results of the simulated pull test were plotted against those conducted on the BOSE machine, and can be seen in Figure 8-4. 


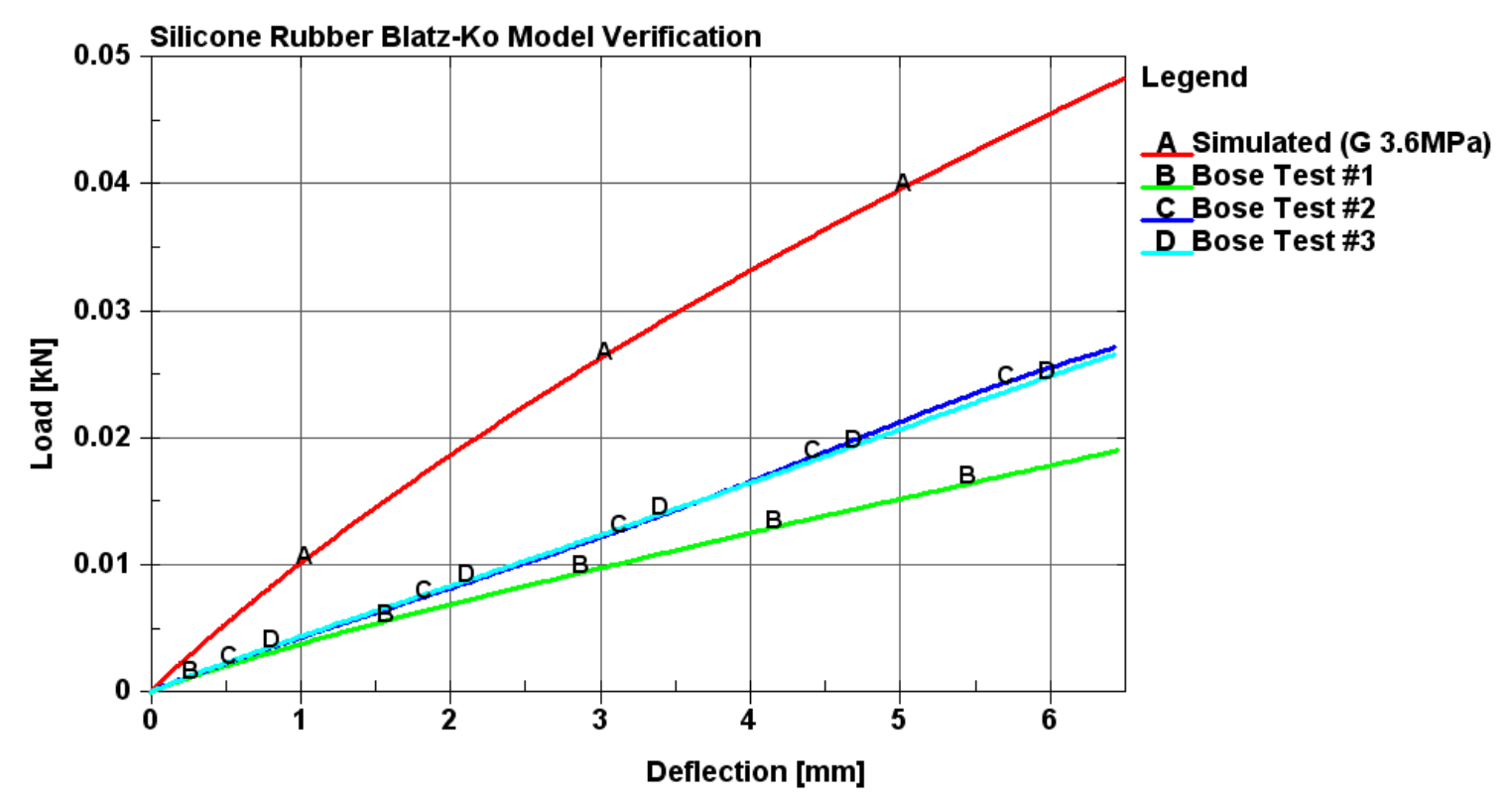

Figure 8-4: Single element pull test load vs. deflection comparison

The results of the simulation have the same loading/deflection trend; however, the loading is approximately double that of the experimental data. This difference most likely can be attributed to the assumed Poisson's ratio, as that is not a variable that can be changed in the 1-D material model. A secondary model was constructed that basically "guessed" at a different value for the shear modulus that would achieve a load displacement curve more similar to that seen in the BOSE tests. A final value of 1.8MPa, approximately half that of the measured value, achieved an appropriate load curve, shown in Figure 8-5. 


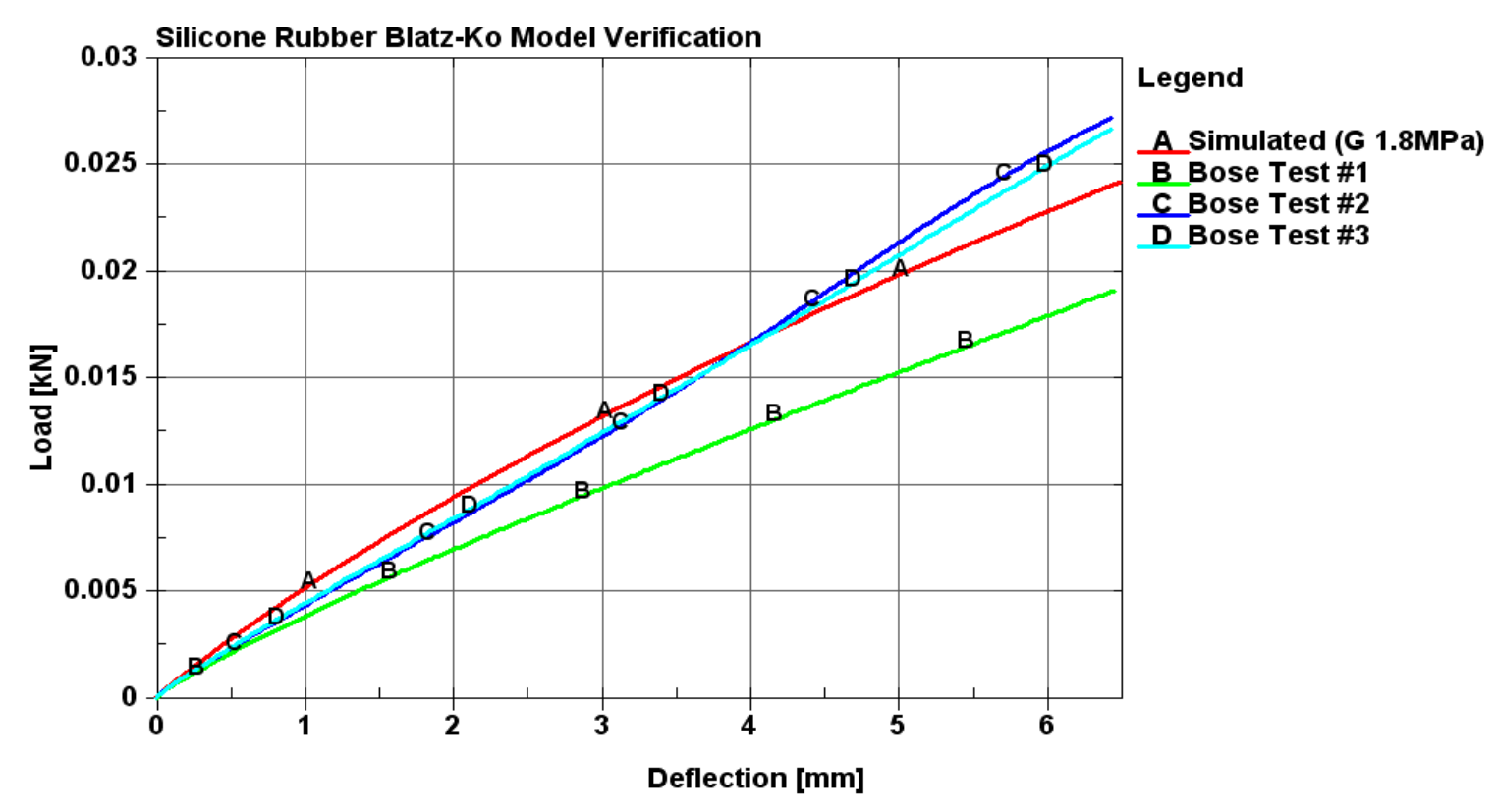

Figure 8-5: Modified shear modulus load/deflection curve comparison

At small local deflections, the load-deflection curve using the original shear modulus is an acceptable approximation of true behavior. As a relatively fine mesh has been selected for the actual FE simulation, large local deflections will not be present, and therefore it was decided to utilize the measured shear modulus of 3.6MPa.

\subsection{Finite Element Simulation}

The aim of this FE analysis is to develop a correlation between the change in internal dimensions of a cylindrical silicone tube and a given bend radius. The two primary dimensions of a tubing are its inside diameter and the wall thickness. The FE model is a dual-parameter study, consisting of five different inside diameters, and four different wall thicknesses, yielding a study size of 20 different arrangements. A summary of all the different parameters can be seen in Table 8-1. 
Table 8-1: Parameter combinations for the silicone rubber FE study

\begin{tabular}{|c|c|c|c|c|}
\hline Diameter & $\mathrm{T}_{1}$ & $\mathrm{~T}_{2}$ & $\mathrm{~T}_{3}$ & $\mathrm{~T}_{4}$ \\
\hline$[\mathrm{mm}]$ & {$[\mathrm{mm}]$} & {$[\mathrm{mm}]$} & {$[\mathrm{mm}]$} & {$[\mathrm{mm}]$} \\
\hline 13 & 0.5 & 1 & 1.5 & 2 \\
\hline 16 & 0.5 & 1 & 1.5 & 2 \\
\hline 19 & 0.5 & 1 & 1.5 & 2 \\
\hline 22 & 0.5 & 1 & 1.5 & 2 \\
\hline 25 & 0.5 & 1 & 1.5 & 2 \\
\hline
\end{tabular}

All of the different simulations are set up like a traditional fixed-end cantilever beam problem, have a tubing length of $100 \mathrm{~mm}$. The bending of the tube occurs in the XZ-plane with the application of a follower force perpendicular to the free end of the beam. The force was varied for each of the different thicknesses so that some form of failure (i.e., kinking or collapse) was apparent. The simulation structure is shown in Figure 8-6.

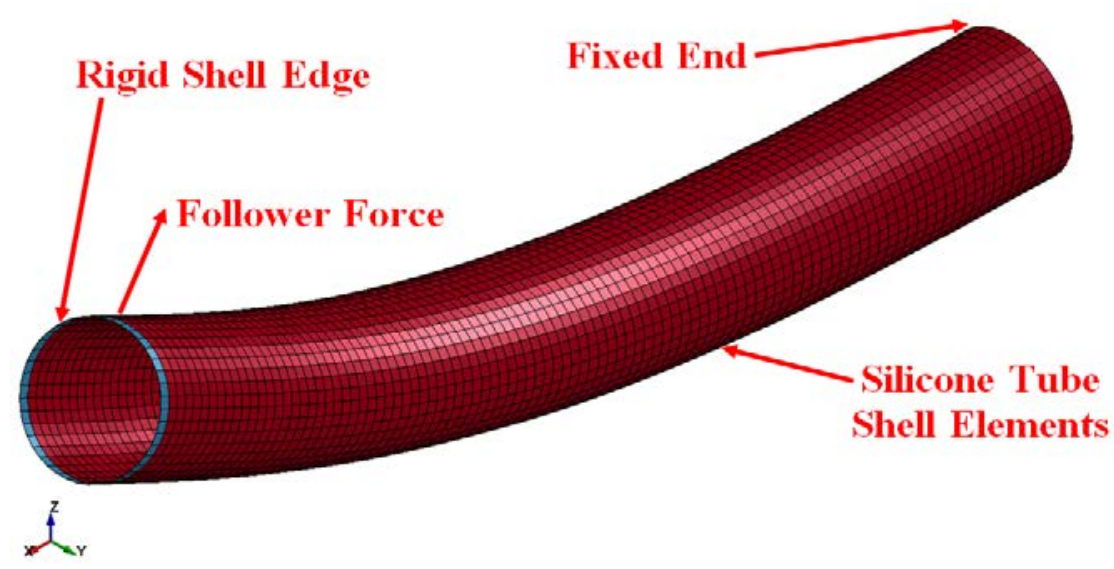

Figure 8-6: Shell element silicone tubing model

The model is constructed of Belytschko-Tsay shell elements with three integration points through the cross section. The element size of $1 \mathrm{~mm} \times 1 \mathrm{~mm}$ is consistent for all 20 models. The silicone tubing is made of Blatz-Ko rubber, and the free end of the tube has a ring of rigid elements. The follower force is applied to the rigid ring to more evenly distribute the loading. The row of nodes closest to the fixed end is constrained such that 
the nodes are not allowed to translate or rotate in any direction. The simulation takes place over a period of $10 \mathrm{~ms}$. The point of greatest interest on the tubing is wherever the largest change in dimensions occurs (i.e., the failure point). This location was determined visually on each of the 20 models by running a preliminary simulation, and representative nodes were selected for tracking during the final simulation.

\subsubsection{Bend Radius Evaluation}

Initially, the bend radius of the cantilever tubing could not be determined directly from the simulation. Using the Euler-Bernoulli beam theory [8-8], a relationship between the bending moment and beam curvature exists for long isotropic beams. The curvature of the beam, $\kappa$, can be calculated using the first and second derivatives of the beam deflection at any given point, $\mathrm{x}$.

$$
\kappa=\frac{\frac{P}{E I}(L-x)}{\left[1+\left[\frac{P X}{2 E I}(2 L-x)\right]^{2}\right]^{3 / 2}}
$$

Although a good approximation, the function does not provide an accurate representation of curvature for hyper-elastic materials, such as silicone rubber. The alternate, more accurate solution can be obtained using Elliptic Integrals [8-8], but due to the iterative numerical solution requirements, a more direct method of measuring the bend radius is desirable. During the simulation, there were ten nodes spaced evenly along the tubing, at its longitudinal neutral axis, whose positions were tracked. Using this position information, a post-processing script was written such that a circle was fit to the effective arc created by the bending tubing. The circle was fit at every time-step, such that a direct correlation could now be constructed between bend radius and the changing dimensions of the tubing. An example of this circle fit is shown in Figure 8-7. 


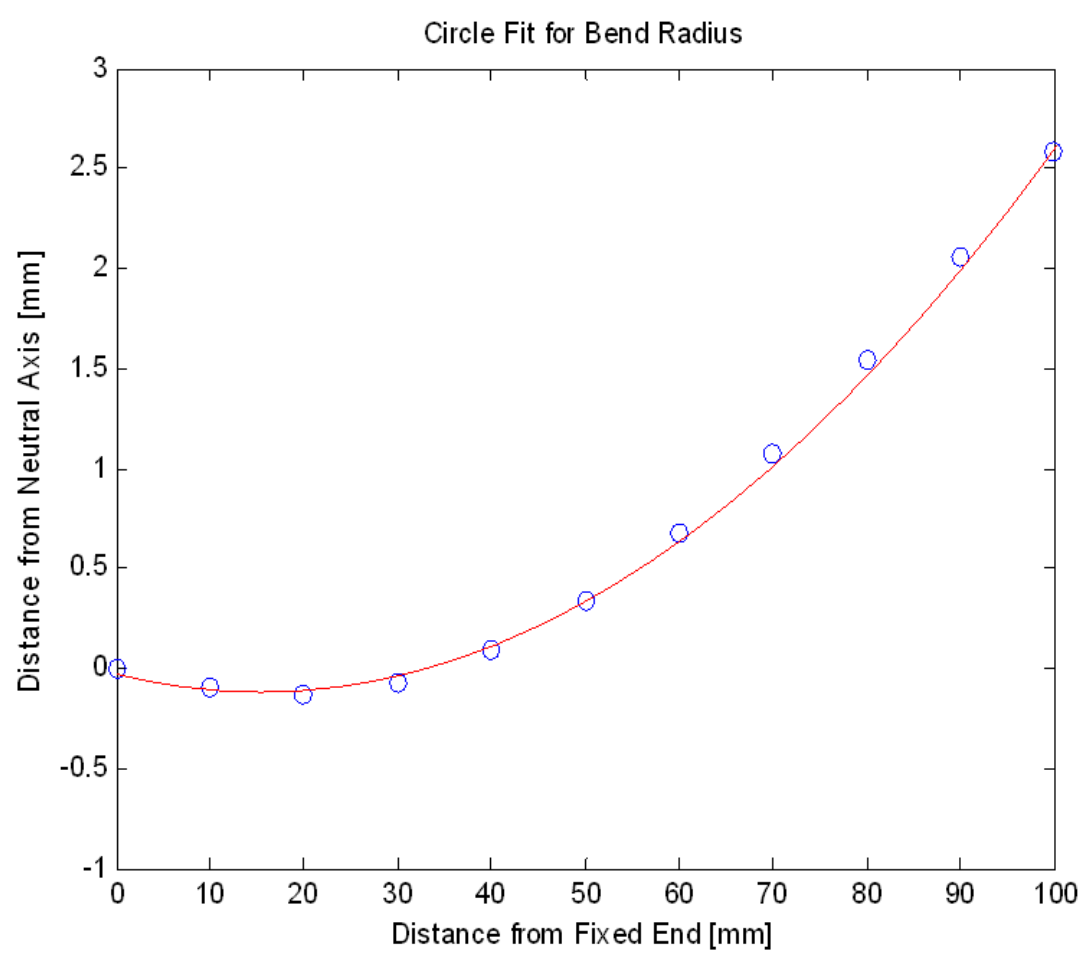

Figure 8-7: Example of the circle-fit function through points on the neutral axis

\subsection{Results}

Each of the 20 trials used one massively parallel processor (MPP), and all executed in approximately 30 seconds. The original approach of this study was to develop a relationship for the change in the internal tubing dimensions based on the tubing thickness, tubing diameter and bend radius. This approach was based on the assumption that all of the tubing would bend at a single cross-section. After examining the output of all twenty trials, there were actually three different bending modes, a ripple, a single kink and a total tubing collapse, as shown in Figure 8-8.
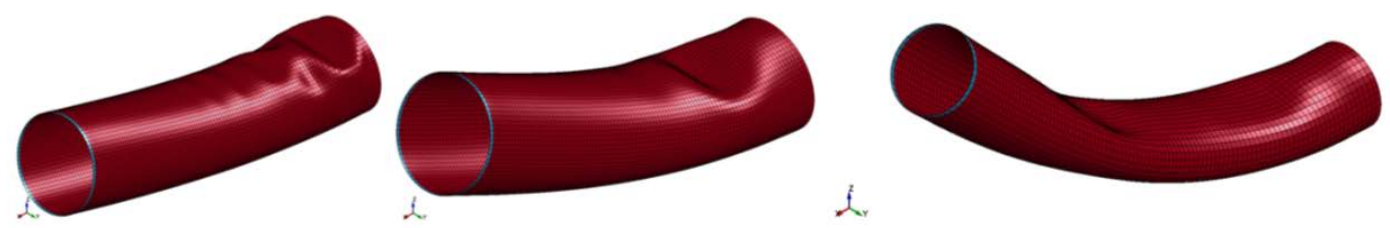

Figure 8-8: Tubing bending modes: ripple, a single kink and total collapse (left to right) 
Also, when the tubing did kink in a single location, the location along the length of the tubing changed as tubing thickness changed, up to a threshold. The location of kink moves asymptotically towards the fixed end as thickness is increased, as shown in Figure 8-9.

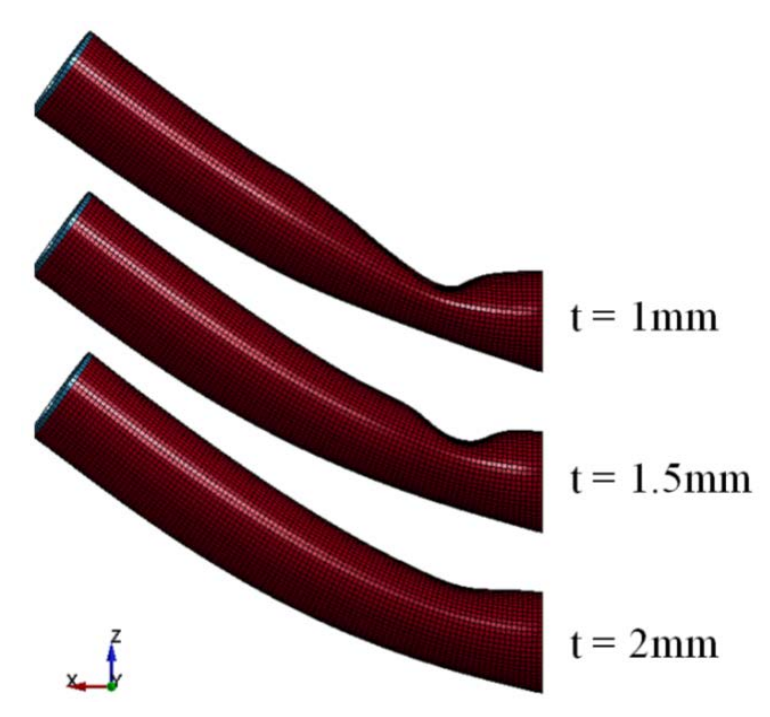

Figure 8-9: Kink point moving asymptotically toward the fixed end

As there are so many different modes of bending and kinking locations, this analysis will focus on the development of general guidelines for how a given tubing thickness and diameter will behave under bending especially right before failure (i.e., kinking), rather than on the development of analytical expressions for the behavior. Table 8-2 summarizes the bending mode(s) and failure point bend radii for all of the simulated models. 
Table 8-2: Bending mode behavior for all models

\begin{tabular}{|c|c|c|c|c|}
\hline $\mathrm{T}=0.5 \mathrm{~mm}$ & Max Bend Radius & Ripple & Kink & Collapse \\
\hline$\varnothing 13$ & 141.62 & $\mathrm{~N}$ & $\mathrm{~N}$ & $\mathrm{Y}$ \\
$\varnothing 16$ & 203.81 & $\mathrm{Y}$ & $\mathrm{N}$ & $\mathrm{Y}$ \\
$\varnothing 19$ & 245.3 & $\mathrm{Y}$ & $\mathrm{N}$ & $\mathrm{Y}$ \\
$\varnothing 22$ & 284.72 & $\mathrm{Y}$ & $\mathrm{Y}$ & $\mathrm{N}$ \\
$\varnothing 25$ & 387.93 & $\mathrm{Y}$ & $\mathrm{Y}$ & $\mathrm{N}$ \\
\hline $\mathrm{T}=1.0 \mathrm{~mm}$ & Max Bend Radius & Ripple & Kink & Collapse \\
\hline$\varnothing 13$ & 143.47 & $\mathrm{~N}$ & $\mathrm{Y}$ & $\mathrm{N}$ \\
$\varnothing 16$ & 179.06 & $\mathrm{~N}$ & $\mathrm{Y}$ & $\mathrm{N}$ \\
$\varnothing 19$ & 219.07 & $\mathrm{~N}$ & $\mathrm{Y}$ & $\mathrm{N}$ \\
$\varnothing 22$ & 260.76 & $\mathrm{Y}$ & $\mathrm{Y}$ & $\mathrm{Y}$ \\
$\varnothing 25$ & 311.49 & $\mathrm{Y}$ & $\mathrm{Y}$ & $\mathrm{Y}$ \\
\hline $\mathrm{T}=1.5 \mathrm{~mm}$ & Max Bend Radius & Ripple & Kink & Collapse \\
\hline$\varnothing 13$ & 145.15 & $\mathrm{~N}$ & $\mathrm{Y}$ & $\mathrm{N}$ \\
$\varnothing 16$ & 176.38 & $\mathrm{~N}$ & $\mathrm{Y}$ & $\mathrm{N}$ \\
$\varnothing 19$ & 210.2 & $\mathrm{~N}$ & $\mathrm{Y}$ & $\mathrm{N}$ \\
$\varnothing 22$ & 245.93 & $\mathrm{~N}$ & $\mathrm{Y}$ & $\mathrm{N}$ \\
$\varnothing 25$ & 281.65 & $\mathrm{~N}$ & $\mathrm{Y}$ & $\mathrm{N}$ \\
\hline $\mathrm{T}=2.0 \mathrm{~mm}$ & Max Bend Radius & Ripple & Kink & Collapse \\
\hline$\varnothing 13$ & 146.01 & $\mathrm{~N}$ & $\mathrm{Y}$ & $\mathrm{N}$ \\
$\varnothing 16$ & 162.58 & $\mathrm{~N}$ & $\mathrm{Y}$ & $\mathrm{N}$ \\
$\varnothing 19$ & 184.6 & $\mathrm{~N}$ & $\mathrm{Y}$ & $\mathrm{N}$ \\
$\varnothing 22$ & 212.81 & $\mathrm{~N}$ & $\mathrm{Y}$ & $\mathrm{N}$ \\
$\varnothing 25$ & 240.35 & $\mathrm{~N}$ & $\mathrm{Y}$ & $\mathrm{N}$ \\
\hline
\end{tabular}

Looking first at the maximum bend radius of the tubing before failure, some trends become apparent. The maximum bend radius (MBR) increases in the form of a power series as the tubing diameter increases. The MBR also increases in the same fashion as wall thickness decreases. Both of these trends can be seen in Figure 8-10. 


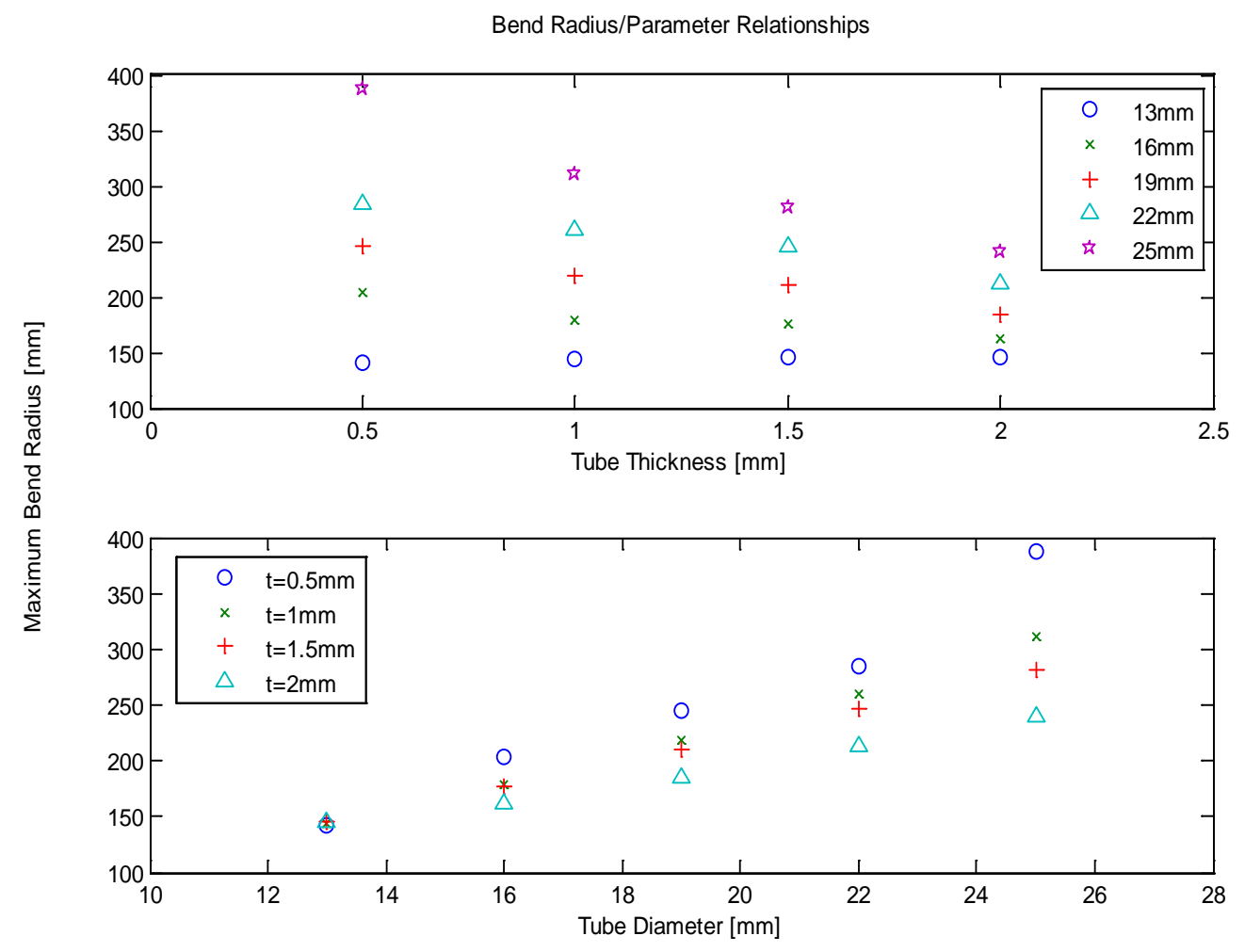

Figure 8-10: Bend radius/parameter relationships

As these trends seem to be inverse of one another, non-dimensionalizing the tubing length with respect to the tubing thickness (i.e., diameter/thickness) could potentially create a linear relationship. As shown in Figure 8-11, this is not the case. The MBR is therefore a function of thickness and diameter, both of which are independent variables. 


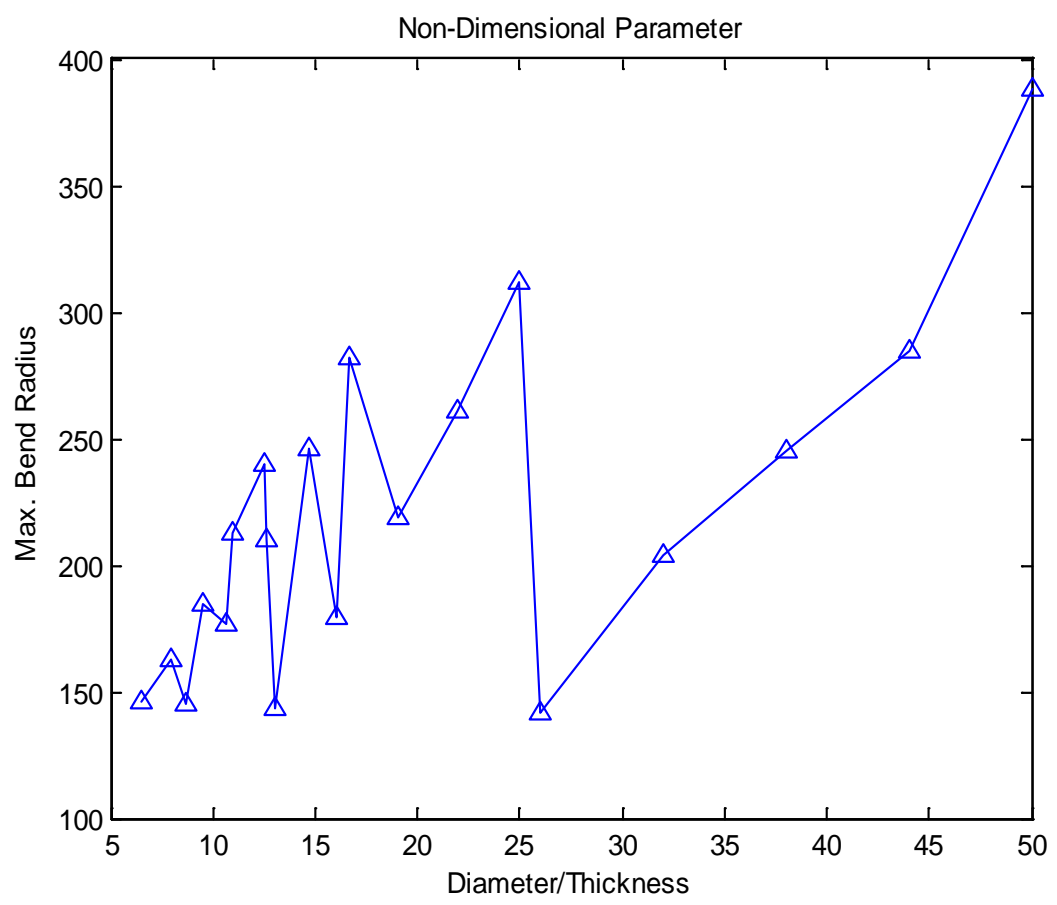

Figure 8-11: Bend radius as a function of non-dimensional diameter/thickness

There is considerable tubing deformation before failure occurs for all twenty models. To analyze the dimensional changes instantaneously, four different points were tracked, representing the dimensional change in two directions, shown in Figure 8-12. The top/bottom contraction is the section of tubing that collapses during bending, and the left/right expansion represents the section of tubing that expands during bending. Both of these displacements are in the same plane through the tubing.

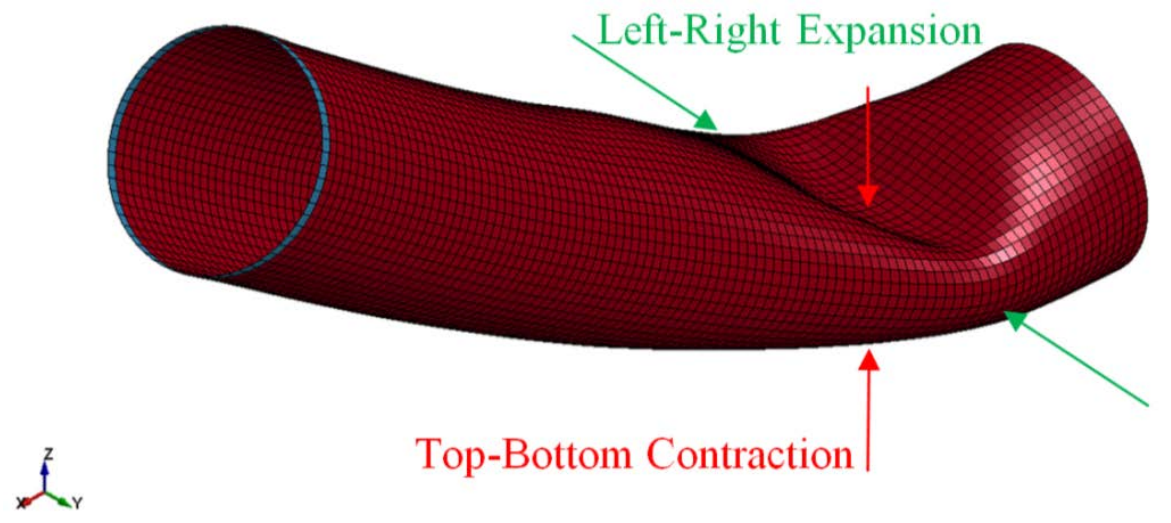

Figure 8-12: Illustration of the dimension change in the plane cross section 
The difference between the left and right points, and the top and bottom points were taken, and plotted against bend radius for every timestep of each simulation, shown in Figure 8-13. These data are a powerful tool, as they prescribe the inner dimensions of the tubing at any given bend radius, from un-deflected up to failure. All of the data have a couple of common trends. As the diameter of the tubing increases, the dimensional change at any point increases. As the wall thickness increases, the dimensional change at any point decreases.
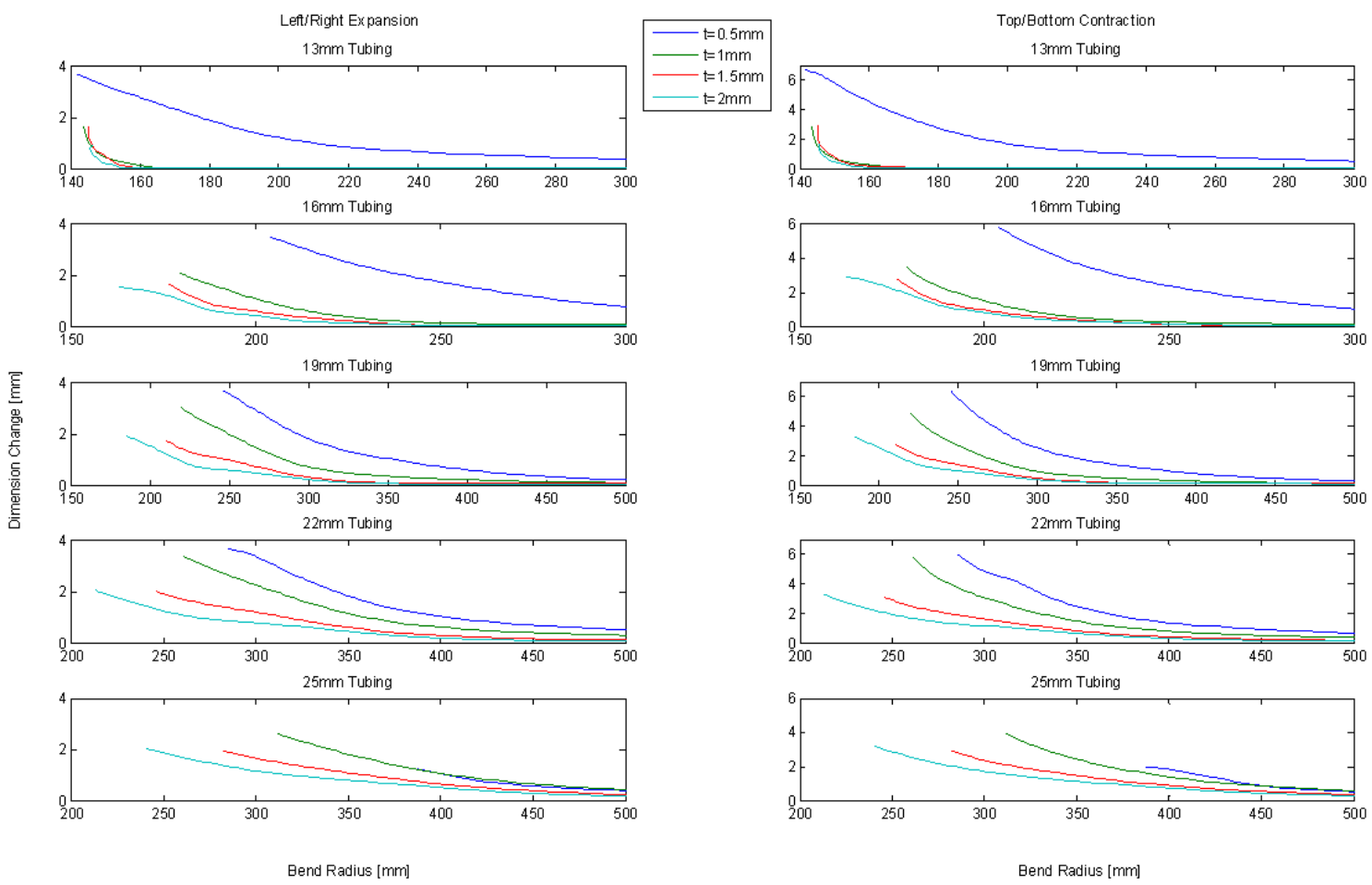

Figure 8-13: Dimensional Changes with respect to instantaneous bend radius

There also appear to be several asymptotes on the trend behavior. As the tubing approaches smaller diameters, the dimensional difference between different thicknesses decreases. As the tubing approaches smaller thicknesses (e.g., $0.5 \mathrm{~mm}$ ), there is a marked increase in dimensional change when compared to greater thicknesses. This behavior may also be attributed to the different bending modes of the tubing. At thin wall 
thicknesses, the mode changes from a single kink to ripple and total tube collapse. The ripple actually adds structural strength to the tubing during bending by distributing the stresses in the tube across a greater area, enabling greater dimensional changes before collapse. A stress distribution comparison can be seen in Figure 8-14.

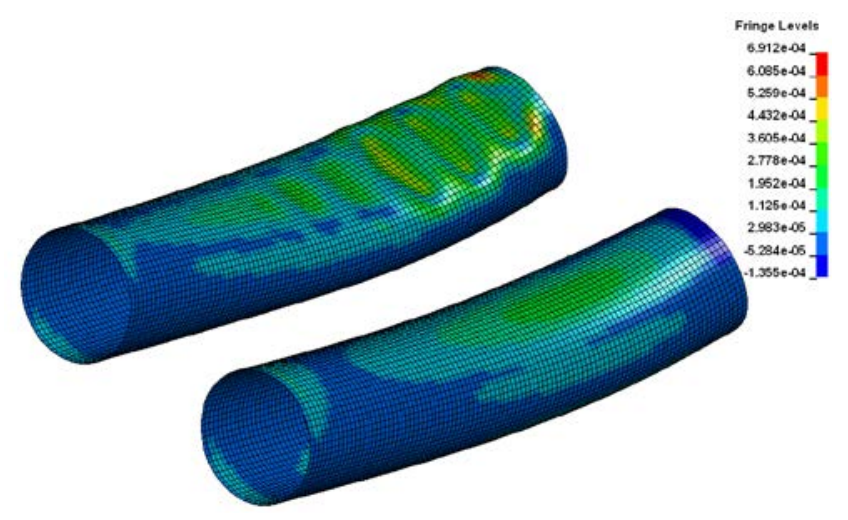

Figure 8-14: Stress distribution comparison between different bending modes

With regard to overtubes in general, this study shows that it may be best to design in the range of diameters and wall thicknesses that seem to exhibit the basic and most common kinking failure behavior. When designing overtubes that have internal supporting structures, such as the MHS, minimizing wall thickness of the overtube may be the best approach. Thin wall tubing seems to exhibit bending behavior that distributes stresses with a ripple failure mode, enabling proper operation of devices within the tubing under a wider range of bending conditions.

The intent of this analysis is to enable designers to make informed decisions when allowing clearances and tolerances for an object passing through a silicone tube. This is directly applicable to many medical devices that utilize silicone overtubes, such as the Material Handling System. This analysis prescribes only basic guidelines for silicone tubing behavior during pure bending and provides a solid basis for additional examination in the future. 


\section{Chapter 9: Conclusion}

In NOTES procedures, in vivo miniature robots can effectively perform surgical tasks. Coupled with this steerable Material Handling System, these robots are given even more flexibility and capability within the peritoneal cavity. The MHS has been designed with maximum functionality without compromising any of the rigid anatomical constraints, and includes a large degree of flexibility for material transport. The MHS performs as intended based on functional requirements as demonstrated in benchtop and porcine in vivo testing. The control method is robust even when pushed beyond the physical constraints of the system. Collectively, the MHS provides a simple, repeatable way for an operator to interface with miniature in vivo robots, improving surgical system flexibility while minimizing impact on the duration of an abdominal surgical procedure.

Through the simulation of a number of different silicone tubing parameter configurations, general recommendations about the tubing behavior were prescribed. These recommendations provide design guidelines for designing a silicone rubber overtube, such as the one utilized in the MHS. With some additional analysis, this FE model of silicone rubber could elucidate a direct analytical representation of the behavior of all compliant silicone overtubes.

In addition to the silicone FE model, the focus of future work should be on refining the finite element models of the spring grasper to optimize the admittance and retention characteristics for diverse payloads. Other work also needs to be done to bring all of the plastic components that cannot be easily sterilized up to sanitary regulations. Also, all of the hardware must be configured for FDA compliance, and the software must be reworked to add redundancies and fail-safes so that it also can be in compliance with 
the FDA. Once FDA compliance is met, testing on a human (cadaver) model, especially focusing on the natural orifice insertion, must be performed. Once all of these additional steps are performed, the MHS could prove to be an essential component in all NOTES procedures involving miniature in vivo robots. These two systems can help make NOTES a more feasible surgical method, eventually replacing MIS procedures as the standard for all abdominal surgical procedures. 


\section{References}

[2-1] Hatzinger, M., 2006. Hans Christian Jacobaeus: inventor of human laparoscopy and thoracoscopy. Journal of Endourology, 20(11):848-850.

[2-2] Horgan, S., Vanuno, D. 2001. Robots in laparoscopic surgery. Journal of Laparoendoscopic \& Advanced Surgical Techniques. Part A, 11(6):415-419.

[2-3] Mack, M. J., 1992. Present role of thoracoscopy in the diagnosis and treatment of diseases of the chest. The Annals of Thoracic Surgery, 54(3):403-8.

[2-4] Kim, V. B., 2002. Early experience with telemanipulative robot-assisted laparoscopic cholecystectomy using da vinci. Surgical Laparoscopy, Endoscopy \& Percutaneous Techniques, 12(1):33-40.

[2-5] Kukleta, J.F., Freytag, C., Weber, M., 2011. Efficiency and safety of mesh fixation in laparoscopic inguinal hernia repair using n-butyl cyanoacrylate: longterm biocompatibility in over 1,300 mesh fixations. The World Journal of Hernia and Abdominal Wall Surgery. American Hernia Society.

[2-6] Ahmed K., Wang T. T., Patel V. M., Nagpal K., Clark J., Ali M., Deeba S., Ashrafian H., Darzi A., Athanasiou T., Paraskeva P. 2011. The role of singleincision laparoscopic surgery in abdominal and pelvic surgery: a systematic review. Surgical Endoscopy. 25(2):378-96.

[2-7] Tracy, C.R., Raman, J.D., Cadeddu, J.A., Rane, A. 2008. Laparoendoscopic single-site surgery in urology: where have we been and where are we heading? Nature Clinical Practice Urology, 5(1): 561-568.

[2-8] Abbott, D.J., Becke, C., Rothstein, R.I., Peine, W.J., 2007, "Design of an Endoluminal NOTES Robotic System," IEEE/RSJ International Conference on Intelligent Robots and Systems, IROS 2007, pp.410-416.

[2-9] ASGE and SAGES. 2006. ASGE/SAGES working group on natural orifice translumenal endoscopic surgery, white paper October 2005. Gastrointestinal Endoscopy, 63(2): 199-203.

[2-10] Giday, S.A., Kantsevoy, S.V., Kalloo, A.N. 2006. Principle and history of natural orifice translumenal endoscopic surgery (NOTES). Minimally Invasive Therapy, 15(6): 373-377.

[2-11] Kalloo, A.N., Sing, V.K., Jagannath, S.B., et al. 2004. Flexible transgastric peritoneoscopy: a novel approach to diagnostic and therapeutic interventions in the peritoneal cavity. Gastrointestinal Endoscopy, 60(1): 114-117. 
[2-12] Rao, G.V., Reddy, D.N., Banerjee, R. 2008. NOTES: human experience. Gastrointestinal Endoscopy Clinics of North America, 18(2): 361-370.

[2-13] Lehman, A.C., Wood, N.A., Dumpert, J., Oleynikov, D., Farritor, S.M., 2008, "Dexterous Miniature in vivo Robot for NOTES," 2nd IEEE RAS \& EMBS International Conference on Biomedical Robotics and Biomechatronics, 2008. BioRob 2008, pp.244-249.

[2-14] Mummadi, R.R., and Pasricha, P.J. 2008. The eagle or the snake: platforms for NOTES and radical endoscopic therapy. Gastrointest Endoscopy, 18:279-289.

[2-15] Jacobs, L.K., Shayani, V., Sackier, J.M. 1997. Determination of the learning curve of the AESOP robot. Surgical Endoscopy, 11(1): 54-55.

[2-16] Ballantyne, G.H. 2002. Robotic surgery, telerobotic surgery, telepresence, and telementoring. Review of early clinical results. Surgical Endoscopy, 16(10): 1389- 1402.

[2-17] Corcione, F., Esposito, C., Cuccurullo, D., Settembre, A., et al. 2005. Advantages and limits of robotassisted laparoscopic surgery: preliminary experience. Surgical Endoscopy, 19(1): 117-119.

[2-18] Satava, R.M. 2002. Surgical robotics: the early chronicles. A personal historical perspective. Surgical Laparoscopy, Endoscopy, and Percutaneous Techniques, 12(1): 6-16.

[2-19] Lanfranco, A.R., Castellanos, A.E., Desai, J.P., Meyers, W.C. 2004. Robotic Surgery: A Current Perspective. Annals of Surgery, 239(1): 14-21.

[2-20] Faraz, A., Payandeh, S., Nagy, A.G. 1995. Issues and design concepts in endoscopic extenders. In Proceedings of 6th IFAC Man-Machine Systems, MIT, 109-114.

[2-21] Swanstrom, L.L., Whiteford, M., Khajanchee, Y. 2008. Developing essential tools to enable transgastric surgery. Surgical Endoscopy, 22(3): 600-604.

[2-22] Mummadi, R.R., Pasricha, P.J. 2008. The eagle or the snake: platforms for NOTES and radical endoscopic therapy. Gastrointestinal Endoscopy Clinics of North America, 18(2): 279-289.

[2-23] Swanstrom, L.L., Khajanchee, Y., Abbas, M.A. 2008. Natural orifice translumenal endoscopic surgery: the future of gastrointestinal surgery. The Permanente Journal, 12(2): 42-47. 
[2-24] Bardaro, S.J., Swanstrom, L. 2006. Development of advanced endoscopes for natural orifice translumenal endoscopic surgery (NOTES). Minimally Invasive Therapy, 15(6): 378-383.

[2-25] Abbott, D.J., Becke, C., Rothstein, R.I., Peine, W.J. 2007. Design of an endoluminal NOTES robotic system. In Proceedings of 2007 IEEE/RSJ International Conference on Intelligent Robots and Systems, San Diego, CA, 410416.

[2-26] Lirici, M.M., Arezzo, A. 2006. Surgery without scars: the new frontier of minimally invasive surgery? Controversies, concerns, and expectations in advanced operative endoscopy. Minimally Invasive Therapy, 15(6): 323-324.

[2-27] Phee, S.J., et al. 2008. Robotic system for no-scar gastrointestinal surgery. International Journal of Medical Robotics, 4(1):15-22.

[2-28] Ota, T., Degani, A., Schwartzman, D., Zubiate, B., McGarvey, J., Choset, H., Zenati, M.A. 2009. A highly articulated robotic surgical system for minimally invasive surgery. Annals of Thoracic Surgery, 87(4): 1253-1256.

[2-29] Chin, W.J., Seow, C.M., Nakamura, A., Head, M., Farritor, S., Oleynikov, D., Nelson, C.A., 2011, "Multifunctional articulating surgical robot for NOTES," Proceedings of the 2011 Design of Medical Devices Conference.

[2-30] Lehman, A.C., Wood, N.A., Dumpert, J., Oleynikov, D., Farritor, S.M., 2008, "Robotic Natural Orifice Translumenal Endoscopic Surgery," IEEE International Conference on Robotics and Automation, ICRA 2008, pp.2969-2974.

[2-31] Rentschler, M.E., Dumpert, J., Platt, S.R., Oleynikov, D., Farritor, S.M., Iagnemma, K., "Mobile in vivo biopsy robot," Proceedings 2006 IEEE International Conference on Robotics and Automation, ICRA 2006. pp.41554160 ,

[2-32] A. C. Lehman, K. A. Berg, J. Dumpert, N. A. Wood, A. Q. Visty, M. E. Rentschler, S. M. Farritor and D. Oleynikov, "Surgery with cooperative robots," Computer Aided Surgery, vol. 13, no. 2, pp. 95-105, 2008.

[2-33] Lehman, A.C., Wood, N.A., Dumpert, J., Oleynikov, D., Farritor, S.M., "Dexterous miniature in vivo robot for NOTES," 2nd IEEE RAS \& EMBS International Conference on Biomedical Robotics and Biomechatronics, BioRob 2008. pp.244-249, 
[2-34] Dumpert, J., Lehman, A.C., Wood, N.A., Oleynikov, D., Farritor, S.M., 2009, "Semi-Autonomous Surgical Tasks Using a Miniature in vivo Surgical Robot," Annual International Conference of the IEEE Engineering in Medicine and Biology Society, EMBC 2009, pp.266-269.

[2-35] Rentschler, M.E., Dumpert, J., Platt, S.R., Oleynikov, D., Farritor, S.M., Iagnemma, K., 2006, "Mobile in vivo Biopsy Robot," IEEE International Conference on Robotics and Automation, ICRA 2006, pp.4155-4160.

[2-36] J. Hawks, Improved mobile wireless in vivo surgical robots: Modular design, experimental results, and analysis, ETD Collection for University of Nebraska Lincoln: Paper AAI3432048, 2010.

[2-37] Platt, S.R., Hawks, J.A., Rentschler, M.E., 2009, "Vision and Task Assistance Using Modular Wireless in vivo Surgical Robots," IEEE Transactions on Biomedical Engineering, Vol.56, pp.1700-1710.

[4-1] Auyang, E., Santos, B., Enter, D., Hungness, E., \& Soper, N., 2011, "Natural Orifice Translumenal Endoscopic Surgery (NOTES): a Technical Review," Surgical Endoscopy Vol. 25, no. 10: pp. 3135-3148.

[4-2] Oleynikov, Dr. Dmitry. Personal Interview. 14 September 2010.

[4-3] Baumeister, Theodore, Ali M. Sadegh, and Eugene A. Avallone. "Friction." Marks' Standard Handbook for Mechanical Engineers. 11th ed. New York: McGraw-Hill, 2007. 3- 2-24. Print.

[5-1] Hribernik, K.A., Ghrairi, Z., Hans, C., Thoben, K.D., 2011, "Co-creating the Internet of Things - First experiences in the participatory design of Intelligent Products with Arduino," 17th Intl. Conference on Concurrent Enterprising, ICE 2011, pp.1-9.

[7-1] Fuller, Janie, Walter Scott, Binita Ashar, and Julia Corrado. Laparoscopic Trocar Injuries: A Report from a U.S. Food and Drug Administration (FDA) Center for Devices and Radiological Health (CDRH) Systematic Technology Assessment of Medical Products (STAMP) Committee. Report. FDA, 2003.

[8-1] "Bose ElectroForce 3200." Bose Worldwide. Bose Corporation. Web. $<$ http://worldwide.bose.com/electroforce/en/web/3200_products/page.html>.

[8-2] Avallone, Eugene A., Baumeister III, Theodore, Sadegh, Ali M. "Mechanics of Materials: Simple Stresses and Strains." Marks' Standard Handbook for Mechanical Engineers. vol. 11. McGraw Hill, 2007. 
[8-3] Madsen, E L, H J Sathoff, and J A Zagzebski. "Ultrasonic shear wave properties of soft tissues and tissuelike materials.” Journal of the Acoustical Society of America. 74.5 1983: 1346-1355.

[8-4] Huang, Yueh-Min Ray. "Ultrasonic Sensing: Fundamentals and Its Applications to Nondestructive Evaluation." Sensors: Advancements in Modeling, Design Issues, Fabrication and Practical Applications. Springer, 2008. 287-304.

[8-5] Folds, D.L. Speed of sound and transmission loss in silicone rubbers at ultrasonic frequencies. The Journal of the Acoustical Society of America, vol. 56, issue 4, p. 1295.

[8-6] Bondi, Scott. "Capturing structural silicone non-linear behavior via the finite element method.” Façade Session - Advanced Architectural Glass Applications. Glass Global, Glass Performance Days. 2009. <www.gpd.fi>.

[8-7] Livermore Software Technology Corporation. LS-DYNA Keyword User's Manual. Volume II: Material Models. Ver. 971, rev. 586. May 20, 2011.

[8-8] O. Kopmaz and Ö. Gündogdu. On the curvature of an euler-bernoulli beam. International Journal of Mechanical Engineering Education, 31:132-142, 2003. 


\section{Appendix}

\section{Appendix A: MHS Insertion w/ Articulated Endoscope}

1. Remove the spring assembly from the overtube assembly

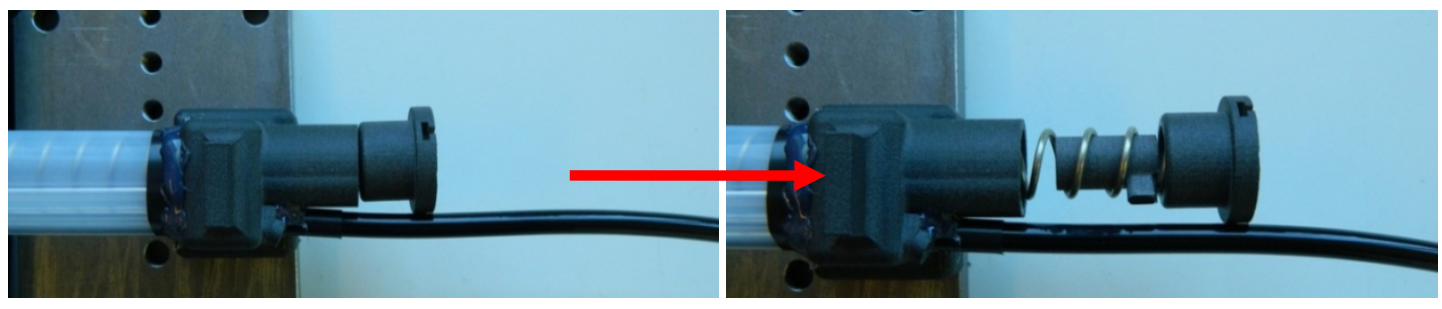

2. Insert an articulated endoscope into the vacated lumen
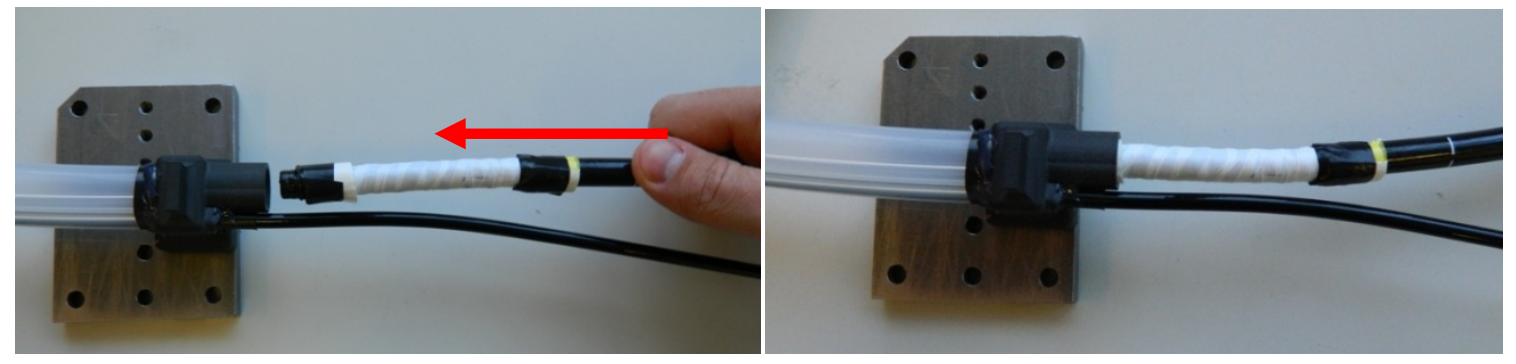

3. The endoscope should be seated in the overtube cap for best articulation

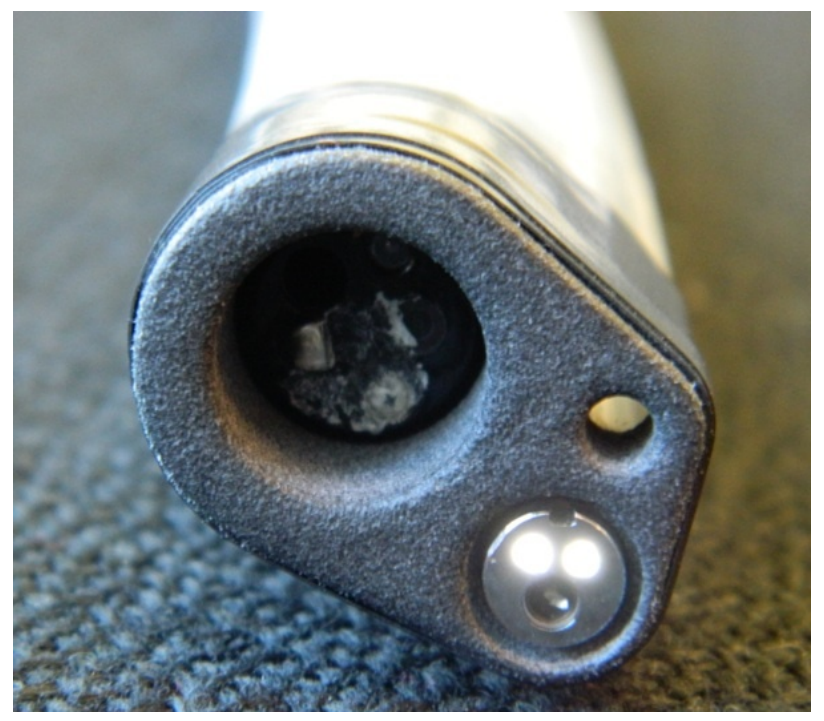


4. Once fully inserted in the esophagus, remove the endoscope and reinsert the spring assembly. Make sure the shuttle is oriented properly and in the fully retracted position against the coupling.

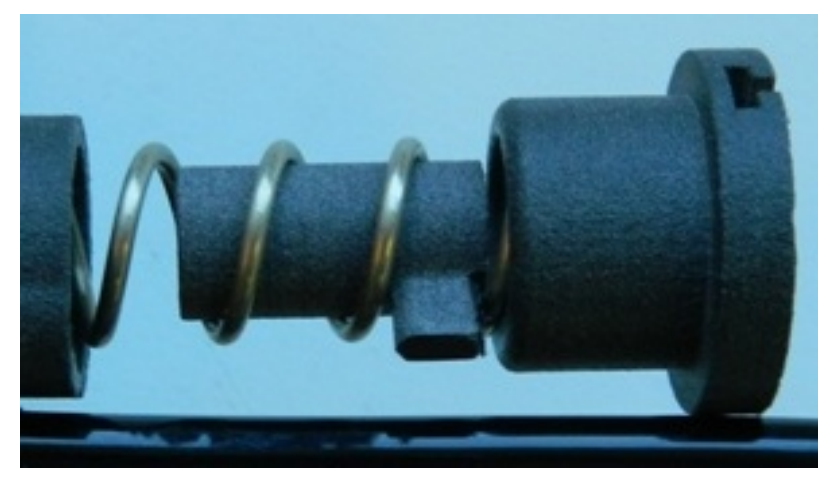

5. Attach the overtube assembly to the control interface by interlocking the keyed couplings. Make sure the overtube mount is seated on the magnetic attachment pads.
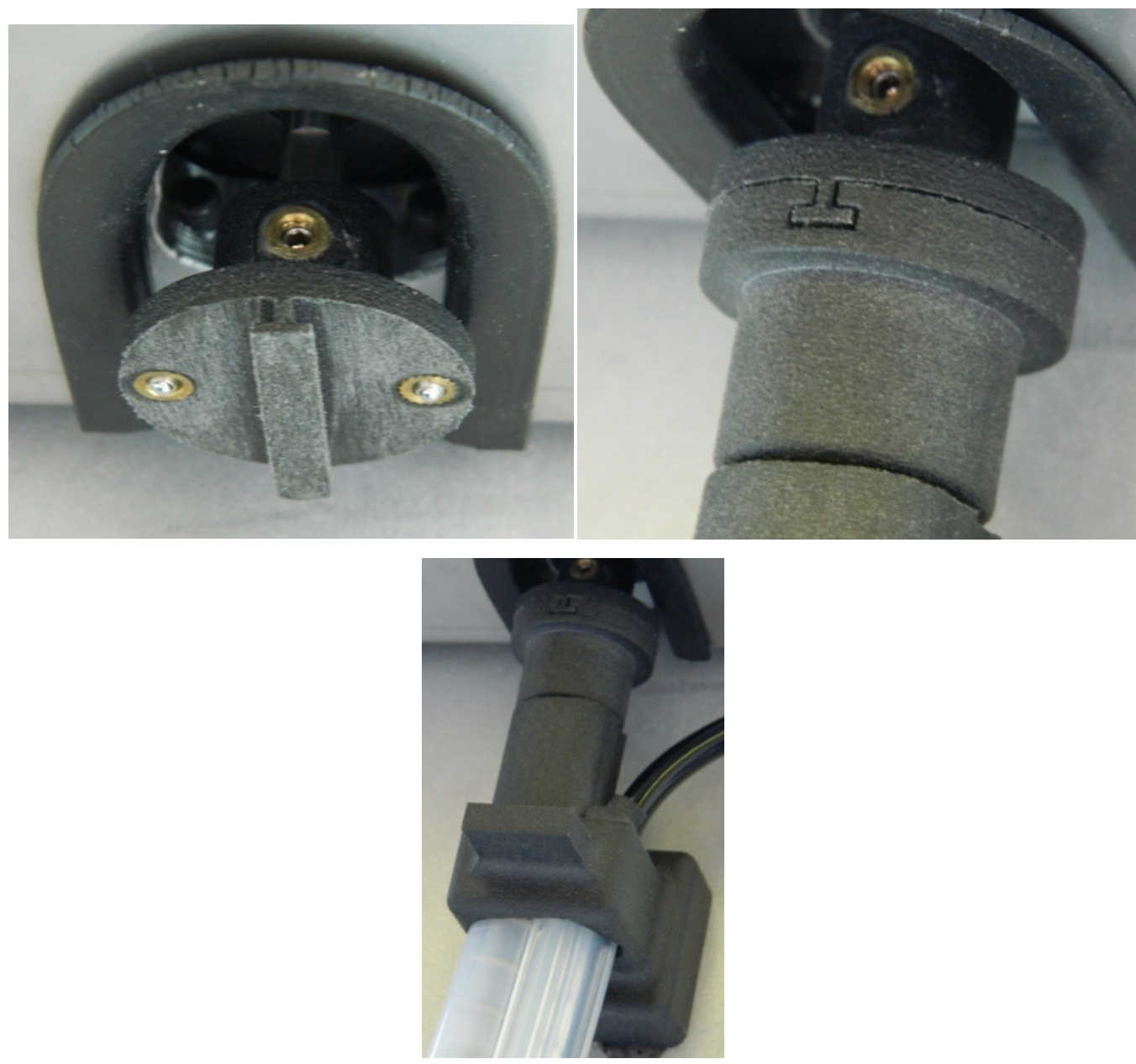


\section{Appendix B: MHS Start-up Procedure \& Control}

1. Plug the control interface into any outlet and the Power LED and Fully Retracted LED will illuminate

2. To calibrate the system, it must be cycled twice before being coupled to the overtube assembly

3. Press the Insert Auto button and allow the system to cycle until the Fully Inserted LED is illuminated

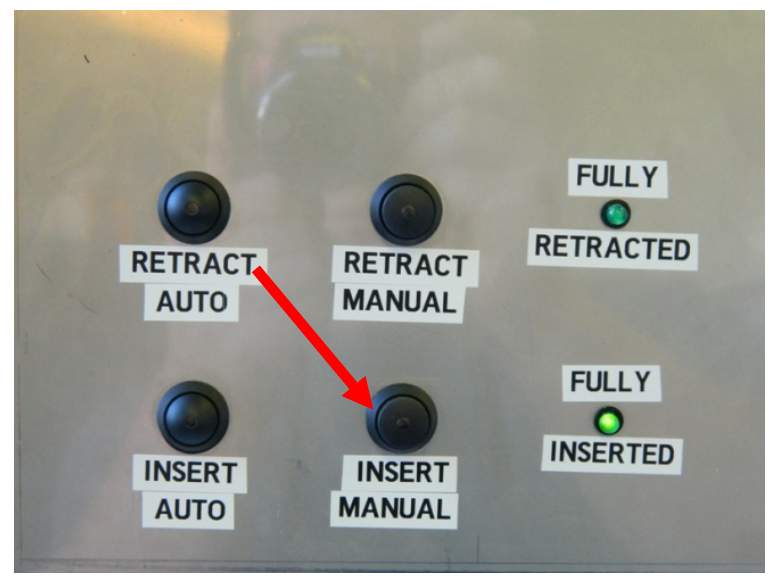

4. Press the Retract Auto button and allow the system to cycle until the Fully Retracted LED is illuminated

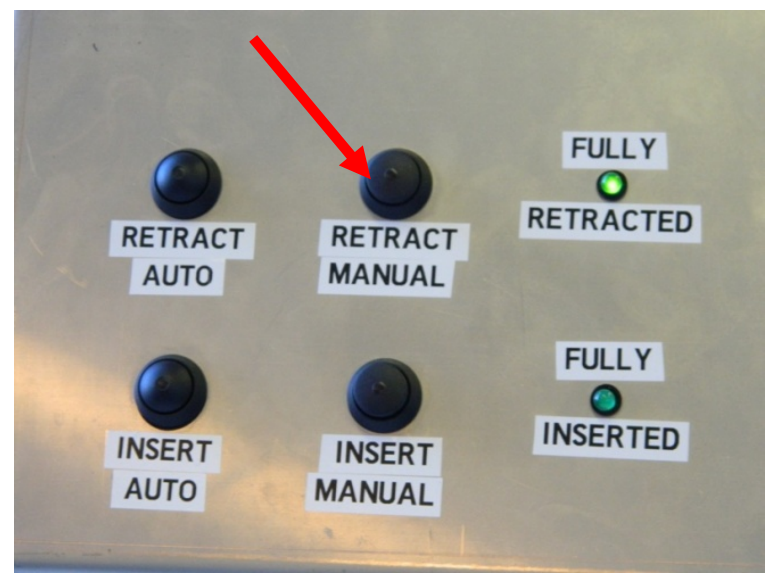


5. Repeat Steps 3-4: the Fully Retracted LED should be illuminated

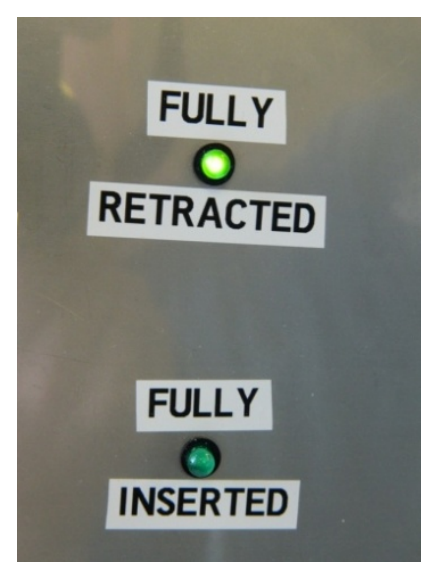

6. Attach the overtube assembly to the control interface by interlocking the keyed couplings. Make sure the overtube mount is seated on the magnetic attachment pads
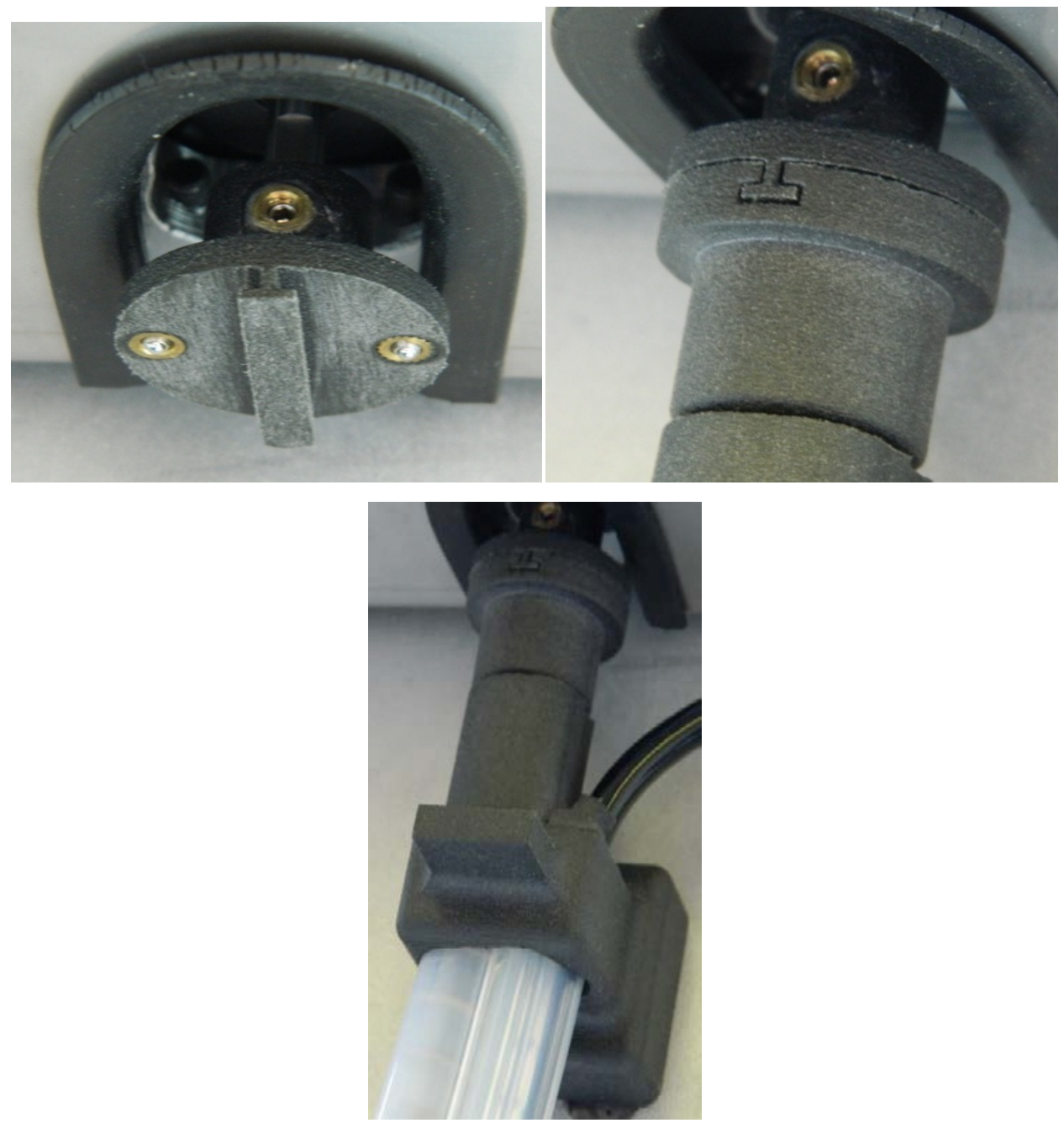


\section{Appendix C: Important Datasheets}

\section{C.1 Low-Friction Coating for Silicone Tubing}

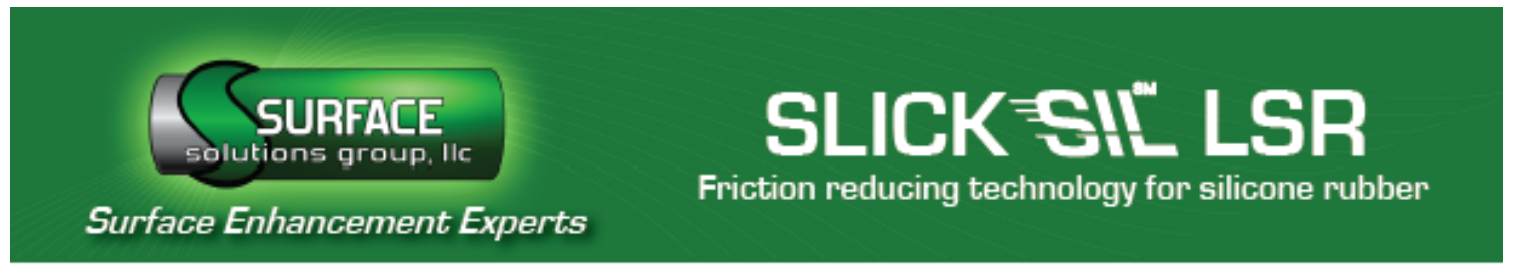

Low Friction \& Flexible Replacement for Parylene

Slick Sil ${ }^{\text {su }}$ LSR is a translucent matte coating designed to reduce the coefficient of friction (COF) and, hence, the friction force of molded and extruded silicone elastomers. The ability of the Slick Sil ${ }^{\text {su }}$ LSR coating system to reduce the surface friction of elastomeric silicone parts enables $\mathrm{LIM}^{*}$ (liquid silicone rubber), HCR, and RTV materials to be utilized in areas that were previously closed to silicones due to their high inherent COF.

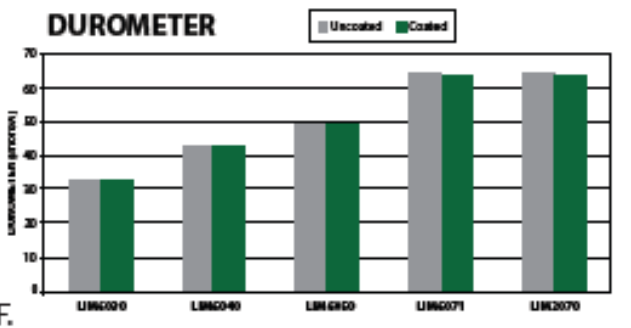

\section{Key Features}

- Low friction (.31) vs. raw silicone (.65)

- Chemical bond

- Thin film (.0005-.001")

- Elastomeric

- Excellent elongation

- Reduces surface dust pick up

- VOC (Volatile Organic Compound) free

- Biocompatible (USP class VI requirements)

- Can be tinted by using silicone compatible pigments

- Anti-microbial properties (optional)

\section{Disadvantages of Parylene \& Silicone Lubricants}

Parylene:

- A rigid coating that cracks on flexible silicone (physical bond)

- Requires time consuming and expensive vapor deposition process

- No anti-microbial properties

Silicone Lubricants:

- If not applied correctly, the lubricant can migrate

- Extra cost and step in the manufacturing process

- Contaminate other areas of manufacturing facility

- Extremely expensive

- No anti-microbial properties
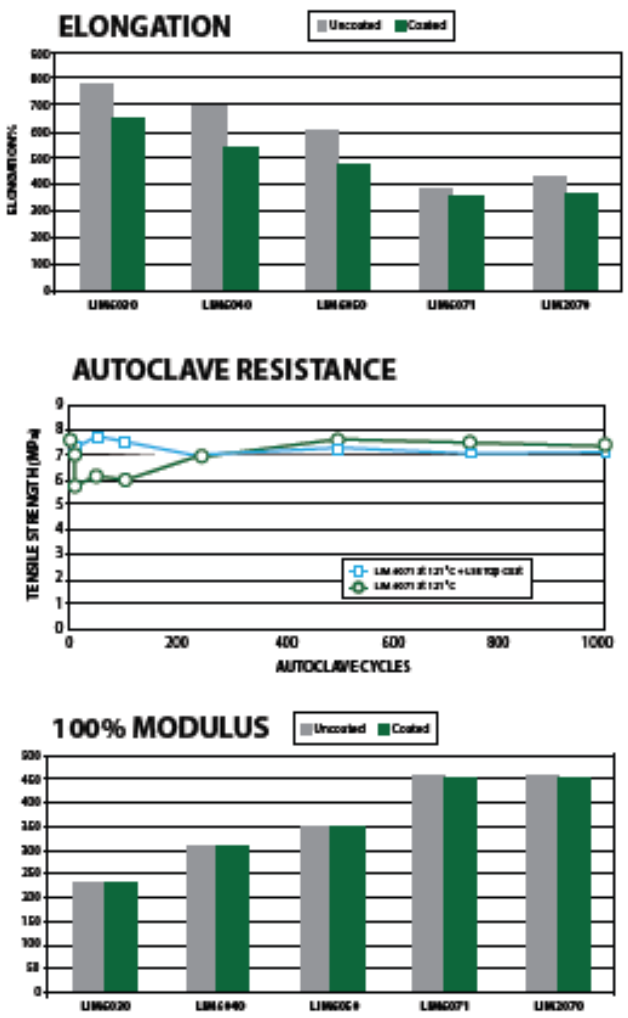


\section{C.2 Stepper Motor}

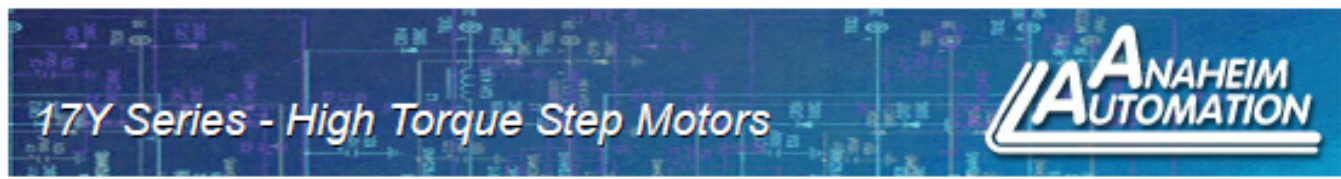

- NEMA 17 Frame Size

- $1.8^{\circ}$ Step Angle (0.9 and $3.6^{\circ}$ also available)

- High Torque - Up to 100 oz-in

- High Step Accuracy and Resolution

- Low Vibration and Noise

- Can be Customized for

-Winding Current

-Shaft Options

- Cables and Connectors

- CE Certified and RoHS Compliant

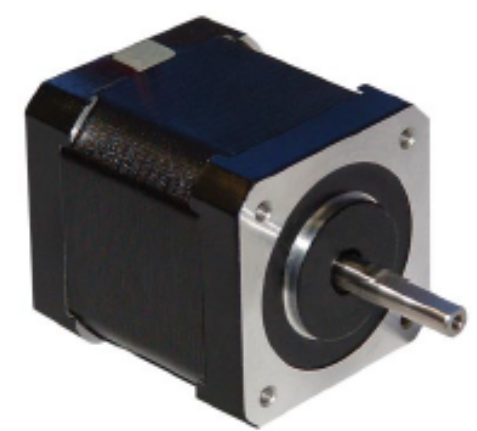

The 17Y Series High Torque Step Motors offer a great value without sacrificing quality. These motors were designed to offer the highest possible torque while minimizing vibration and audible noise. A broad line of motor windings and stack lengths are available off-the-shelf, or the motors can be customized to fit your machine requirements. We can customize the winding to perfectly match your voltage, current, and maximum operating speed for maximum flexability.

See Accessories on this website for optional motor adders, such as encoders, cables and connectors. Gearbox optionals can be found under Gearboxes.

See compatible Drivers for the 17Y Series: MBC15081, and MBC25081TB

\begin{tabular}{|c|c|c|c|c|c|c|c|c|c|}
\hline Model \# & $\begin{array}{l}\text { NEMA } \\
\text { 5ize }\end{array}$ & $\begin{array}{l}\text { Bipolar } \\
\text { Tonque } \\
\text { (oz-in) }\end{array}$ & $\begin{array}{l}\text { Bipolar } \\
\text { Current } \\
\text { (A) }\end{array}$ & $\begin{array}{l}\text { Bipolar } \\
\text { hauctance } \\
\text { (mH) }\end{array}$ & $\begin{array}{c}\text { Robbr } \\
\text { nerfa } \\
\text { (az-in-sect) }\end{array}$ & $\begin{array}{c}\text { Shat } \\
\text { Diameter } \\
\text { (in) }\end{array}$ & $\begin{array}{l}\text { * of } \\
\text { Lead } \\
\text { Wires }\end{array}$ & $\begin{array}{l}\text { Weight } \\
\text { (BS) }\end{array}$ & $\begin{array}{l}\text { L } \\
\text { Lengh } \\
\text { (in) }\end{array}$ \\
\hline $17 Y 0015-L W / 4$ & 17 & 24 & 0.40 & 96 & .00028 & .197 & 4 & 0.33 & 1.02 \\
\hline 17Y1015-LV/4 & 17 & 91 & 0.28 & 49 & .00050 & .197 & 4 & 0.44 & 1.34 \\
\hline $17 Y 1025-L W / 4$ & 17 & 31 & 0.67 & 17 & .00050 & .197 & 4 & 0.44 & 1.34 \\
\hline $17 Y 2015-L W / 4$ & 17 & 50 & 0.28 & 120 & .00076 & .197 & 4 & 0.62 & 1.57 \\
\hline $17 Y 2025-1 W / 4$ & 17 & 50 & 0.85 & 10 & .00076 & .197 & 4 & 0.62 & 1.57 \\
\hline 17Y9015-LW/4 & 17 & 62 & 0.28 & 100 & .00096 & .197 & 4 & 0.77 & 1.89 \\
\hline 17YYSO25-LW/4 & 17 & 62 & 0.85 & 11 & .00096 & .197 & 4 & 0.77 & 1.89 \\
\hline $17 Y 4025-L W / 4$ & 17 & 100 & 0.85 & 29 & .00143 & .197 & 4 & 1.10 & 2.96 \\
\hline
\end{tabular}

Notes: All Shafts have a flat unless otherwise noted. The 7 th character "S" denotes a single shaft, use " $D$ " for double shaft. Double shafts include encoder mounting provisions. Custom cables, connectors, and windings are available upon request.

L010174

910 East Orangefair Ln. Anaheim, CA 92801 Tel. (714) 992-6890 Fax. (714) 992-0471 www.anaheimautomation.com 

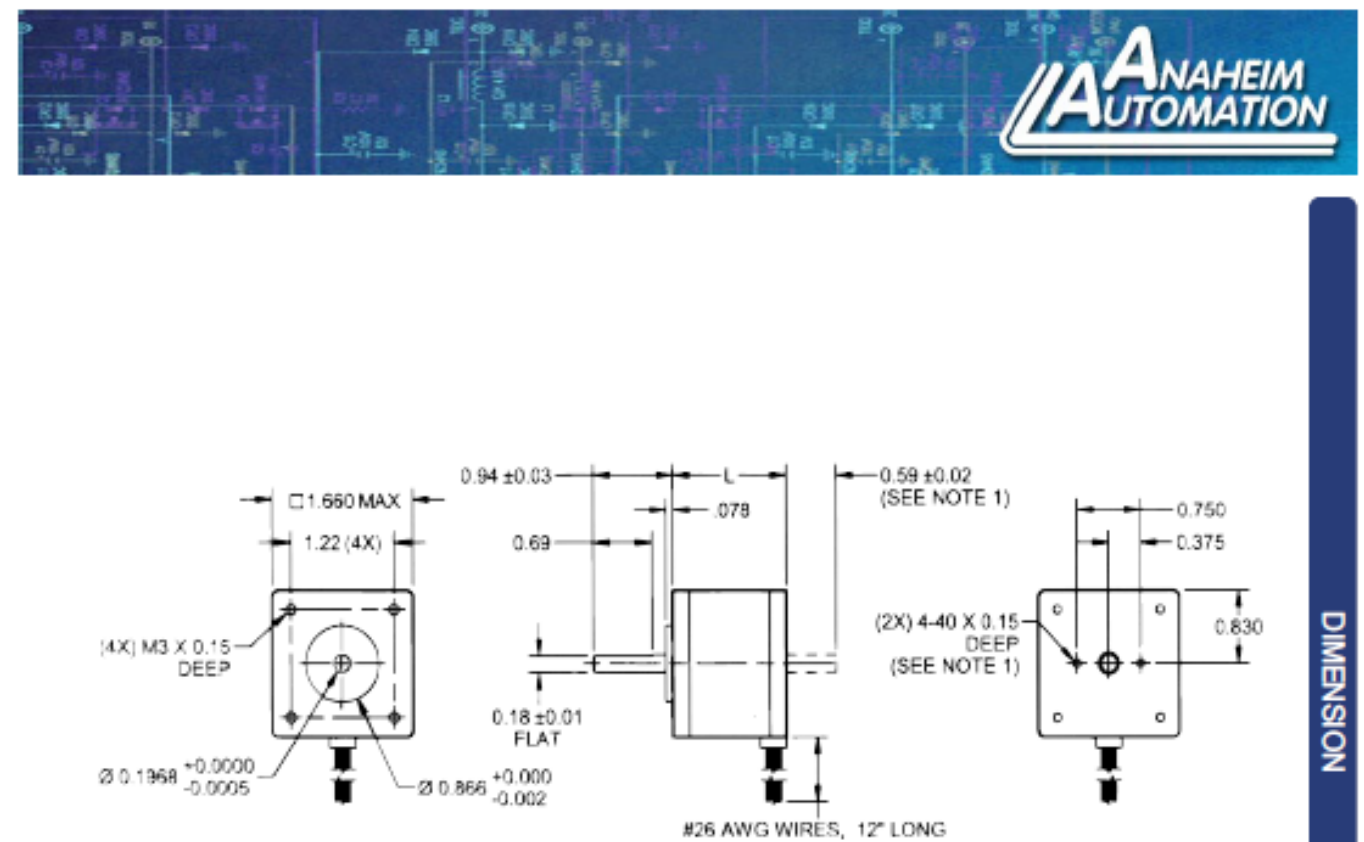

Note 1: Double shaft option only.

(All units are inches)
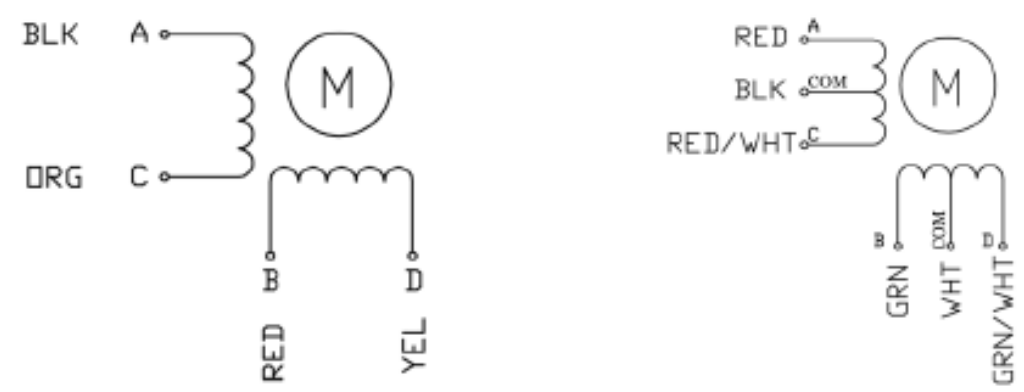


\section{C.3 Stepper Motor Encoder}

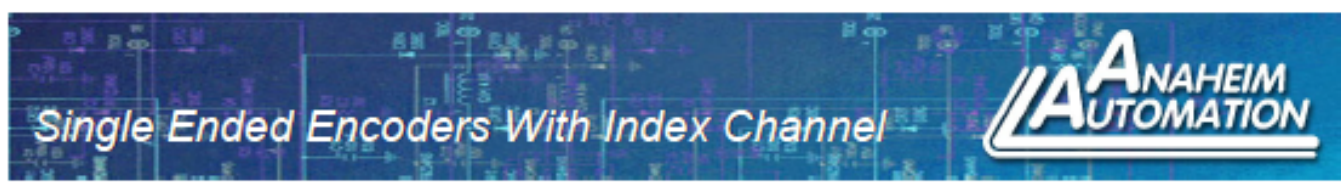

- Small Size, Pre-Mounted to Dual Shaft Motor

-Available CPR: 32, 50, 100, 200, 250, 360, $400,500,720,1000,1024$ and 1250.

- Tracks from 0 to $100,000 \mathrm{cycles} / \mathrm{sec}$

- 2 Channel Quadrature TTL Squarewave Outputs

- Index Channel (3rd channel)

- Accepts +/-0.010" axial shaft play

$-40^{\circ} \mathrm{C}$ to $+100^{\circ} \mathrm{C}$ Operating Temperature

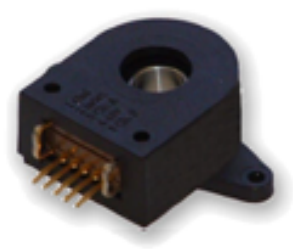

Our Single Ended Encoders with an Index channel are transmissive optical encoder modules. These modules are designed to detect rotary position with a codewheel when added to the end of an Anaheim Automation dual shaft motor. These Single Ended Encoders consist of a lensed LED source and a monolithic detector IC enclosed in a small polymer package. These modules use phased array detector technology to provide superior performance and greater tolerances over traditional aperture mask type encoders. They provide digital quadrature outputs, and come standard with a third index channel output on all resolutions and are capable of sinking or sourcing $8 \mathrm{~mA}$ each. These encoders are powered from a single +5VDC power supply. Also, they are RoHS compliant.

Example: To order an encoder, add a " ${ }^{~ "}$, the CPR number and a SI on the end of any Anaheim Automation dual shaft motor. For example, to place a $1000 \mathrm{CPR}$ encoder on a 23Y106D-LW8, the part number would be 23Y106D-LW8-1000SI.

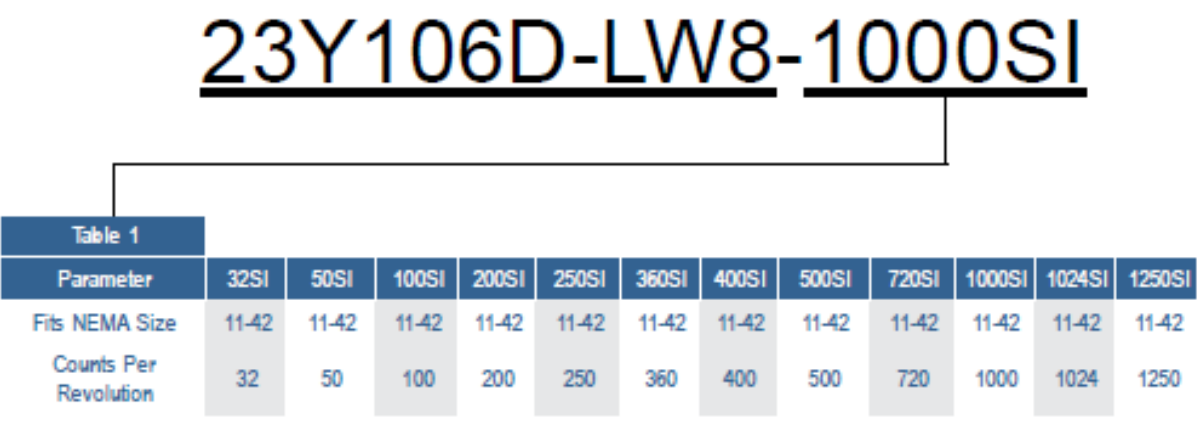
L010390 


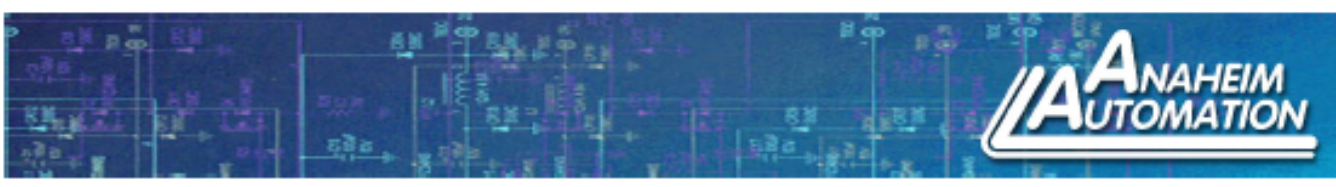

DIMENSIONS
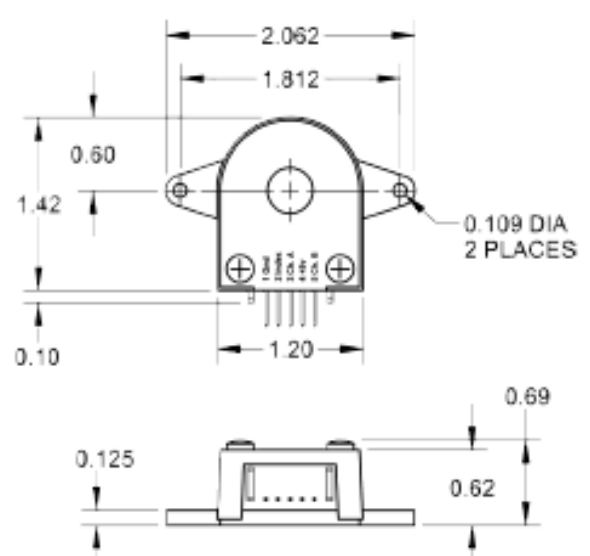

Note: All dimensions are in inches.
SINGLE-END ENCODER PINOUT

TOP OF ENCODER FACING PLUG
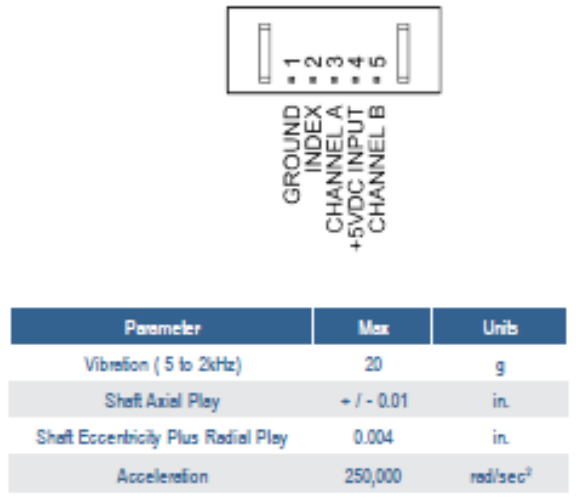

Accelerefon

SINGLE-END ENCODER TIMING DIAGRAMS
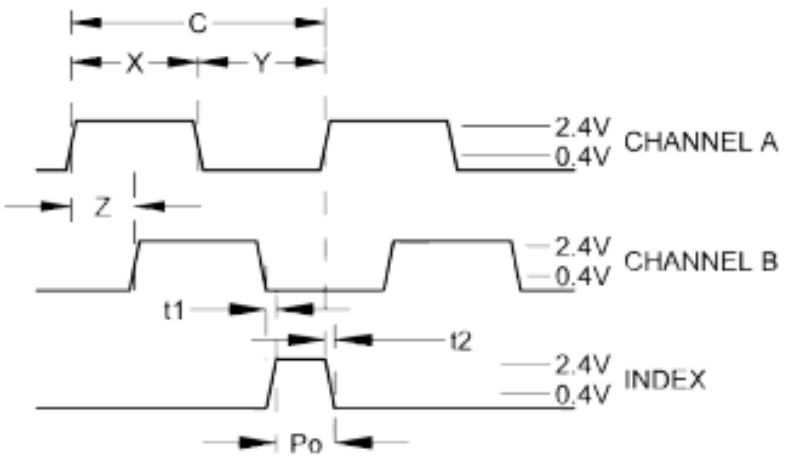

Rotation:

CW - B leads A CCW - A Leads B 

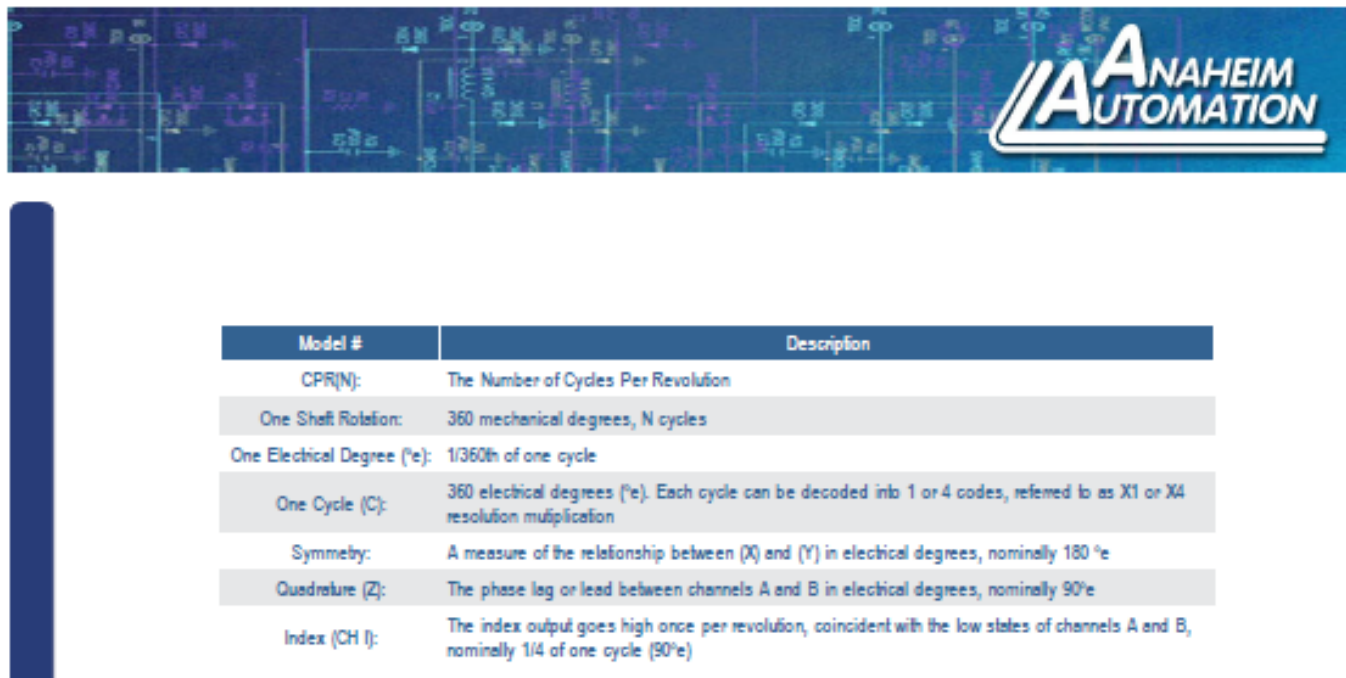

\begin{tabular}{|l|c|c|c|c|}
\hline Paremeter & Min & Typ & Max & Unib \\
\hline
\end{tabular}

Supply Current

32 CPR only

720, 1000, 1024, 1250 CPR Only

All Other Resolutions

$\begin{array}{rrr}- & 27 & 30 \\ - & 55 & 57\end{array}$

\section{.}

Output Low

32, 720, 1000, 1004, 1250 CPR Only

A1 Other Resolstion:

$30 \quad 57 \quad 85$

7 mA

\section{Descriplon \\ Index Pulse Width}

\begin{tabular}{|l|l|l|l|l|}
\hline Symbol & Min & Typ & Max & Unit \\
\hline
\end{tabular}

Output High"

\begin{tabular}{|c|c|c|c|c|}
\hline $32,720,1000,1024,1250$ CPR Only & 20 & - & - & Vots \\
\hline Al Other Resolutions & 24 & - & - & Vols \\
\hline Output Current Per Channel & & & & \\
\hline $32,720,1000,1024,1250$ CPR Only & -8.0 & - & 8.0 & $m A$ \\
\hline Ai Other Ressolutions & -10 & - & 5.0 & $\mathrm{~mA}$ \\
\hline
\end{tabular}

All Resolutions

Ch. I Rise After Ch. B or Ch. A Fal

$32,720,1000,1024,1250$ CPR only

All Oter Resolufons

Ch. I Fall After Ch. A or Ch. B Rise

$32,720,1000,1024,125$ CPR only

All Oter Resolufions

$\begin{array}{ccccc}\text { Po } & 60 & 90 & 120 & \text { = } \\ \text { t1 } & 10 & 100 & 250 & \mathrm{~ns} \\ \text { t1 } & -300 & 100 & 250 & \mathrm{~ns} \\ & & & & \\ \text { E } & 70 & 150 & 300 & \mathrm{~ns} \\ \text { t2 } & 70 & 150 & 1000 & \mathrm{~ns}\end{array}$

\section{Recommended Openefing Condifons}

Min

\begin{tabular}{|c|c|}
\hline Max & Unib \\
\hline 100 & ${ }^{\circ} \mathrm{C}$ \\
\hline 5.5 & Vols \\
\hline 100 & pF \\
\hline 100 & $\mathrm{kHtz}$ \\
\hline
\end{tabular}

\begin{tabular}{|c|c|}
\hline Cable Ordering th & Length \\
\hline CBL-AA4032 & $1 \mathrm{ft}$ \\
\hline CBL-AAAOS2-04 & $4 \mathrm{ft}$ \\
\hline CBL-AAADS2-10 & $10 \mathrm{ft}$ \\
\hline
\end{tabular}

"Unloaded high level ouput voltage is $4.80 \mathrm{~V}$ typically. $4.2 \mathrm{~V}$ minimum. 


\section{C.4 Power Supply}

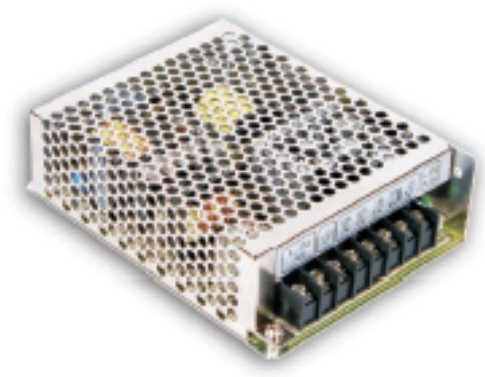

Features :

- Universal AC nput/Full range

- Protections:Short circuiv Over load Over voltage

- Cooling by tree air corrvection

- LED indicator for power on

- $100 \%$ full load burn in test

- All using 105'C longlife electrolycic capacitors

- Withstand 300VAC surge input for 5 second

- High operating temperature up to $70 \mathrm{C}$

- Withstand SG vibration test

- High etticiency, bng life and high relability

- 3 years warranty

\begin{tabular}{|c|c|c|c|c|c|}
\hline \multicolumn{2}{|l|}{ MOOEL } & \multicolumn{2}{|l|}{ RD-65A } & \multicolumn{2}{|l|}{$20-658$} \\
\hline \multirow{12}{*}{ OUTPUT } & OUTPUT NUMBER & $\mathrm{CH} 1$ & $\mathrm{CH}_{2}$ & C서1 & $\mathrm{CH}_{2}$ \\
\hline & DC VOLTAGE & SW & $12 \mathrm{~V}$ & F & $24 \mathrm{~V}$ \\
\hline & RATED CU RSENT & 64 & $3 \mathrm{~A}$ & 44 & $2 \mathrm{~A}$ \\
\hline & CURRE NT RANGE & $0.3-3$. & $0.2-4 A$ & $0.3-84$ & $0.2-34$ \\
\hline & RATED POWER & siw & & बw & \\
\hline & APPLES NasE $(\max )$ Nota. & $30 \mathrm{~m} / \mathrm{p} \cdot \mathrm{p}$ & $120 \mathrm{mWp} \cdot \mathrm{p}$ & $30 \mathrm{~m} / \mathrm{Vp} \cdot \mathrm{p}$ & $150 \mathrm{ml} / \mathrm{p} p$ \\
\hline & VOLTAGE ADS.RANGE & \multicolumn{2}{|l|}{ 어t:4J5-5.5V } & \multicolumn{2}{|c|}{ CHEA.T5-5.SW } \\
\hline & VOLTA \&E TOLERANCENCLS 3 & $12.0 \%$ & $16.0 \%$ & $2.0 \times$ & $+4-6 \%$ \\
\hline & LNEREQULATION NOLA & $10.5 \%$ & $\pm 1.5 \%$ & $0.5 \mathrm{~K}$ & $120 \%$ \\
\hline & LOA D REGULATION Nota.S & $20.5 \%$ & $\pm 30 \%$ & $70.5 \mathrm{~K}$ & $\pm 40 \%$ \\
\hline & SETUP, NISE TIME & so0ma, 20ma/23ONAC & $10 \mathrm{ma}, 30 \mathrm{ma}$ & & \\
\hline & HOLO TME (TYP.) & \multicolumn{4}{|c|}{ 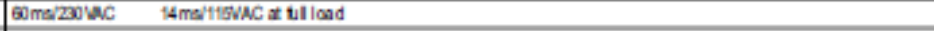 } \\
\hline \multirow{6}{*}{ NPUT } & VOLTA GE RANGE & \multicolumn{4}{|c|}{ 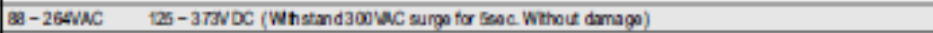 } \\
\hline & FREQUENCY RANCE & \multicolumn{4}{|c|}{$47-63+2$} \\
\hline & EFACENCY(TYP.) & \multicolumn{2}{|c|}{796} & \multicolumn{2}{|l|}{786} \\
\hline & AC CURRENT (Typ.) & \multicolumn{4}{|c|}{ 2NH1ENAC 1.2N2BONAC } \\
\hline & INRJSH CUR RENT (TYR) & \\
\hline & IEAXAGE CURRENT & \multicolumn{4}{|c|}{$2 \mathrm{~mA} / 2 \operatorname{sov} \mathrm{AC}$} \\
\hline & & $110-160 \%$ rand atp & & & \\
\hline PROTETIO & OVERLOLD & Protioc Ion typo: Hiloou & recovorsaut & condtion be & \\
\hline Prolection & & 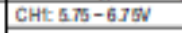 & & & \\
\hline & ONERVOLTADE & Protioc Ion typo: Hibou & rocovorsaut & condtion be & \\
\hline & WORNAG TEN?. & $-25-+\pi 0<$ Ratar too & d dorating 0 & & \\
\hline & WORNAS HUMOITY & $20-90 \times$ RHA non-ond & & & \\
\hline BWponert & STORAGETENP, HUMIOTY & $-20-+26 C_{0}-10-968$ & & & \\
\hline & TEMP COEFFICIENT & $\pm 0036 /(0)(0-301) 0$ & uput & & \\
\hline & ИBeATION & $10-500 \mathrm{~Hz}, 3 \mathrm{G} 10 \mathrm{~min}$ & peribdfor 6 & $1.2 \operatorname{axces}$ & \\
\hline & SAFETY STAND AQDS & U6OOSO-1, TUV ENG0 & provod & & \\
\hline & \begin{tabular}{|l} 
WTHST AND VOLTAGE \\
\end{tabular} & IP-ONPZJWVAC MPF & MC OPA & & \\
\hline SAFETYS & ISOATION RE SSTANCE & P-ONP, IPFG, OP.P & Chmelsong & & \\
\hline EMC & EM COAOUCTION S RACIAT TOA & Complanco b ENGCO & (222) Clase 8 & & \\
\hline hater) & HARNONC CURRENT & Complinos b EN6100 & & & \\
\hline & ENSINYJNTY & Complanos b EN6100 & 4.5.8.1t:E & 6.2 (EN 300 & bud, cintia A \\
\hline & MTBF & 205 .9Ghrsmin. ML. & $\left.11 \pi(25)^{\circ}\right)$ & & \\
\hline OTHERS & DIMENSION & $1298739 m m$ (L"WF & & & \\
\hline & PACXNG & $0.446 \mathrm{~g}^{2} 30 \mathrm{pe} / \mathrm{/4} .2 \mathrm{Kg}$ & & & \\
\hline NOTE & 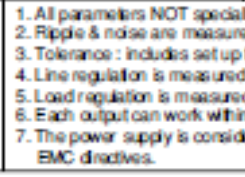 & 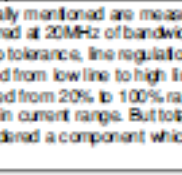 & 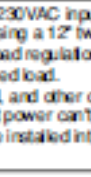 & $\begin{array}{l}\text { 5ic d ant } \\
\text { inasd wh } \\
\text { bad } \\
\text { thener. } \\
\text { The fral s }\end{array}$ & El capactor. \\
\hline
\end{tabular}

Tel: 1-973-779-8282 www.trcelectronics.com 

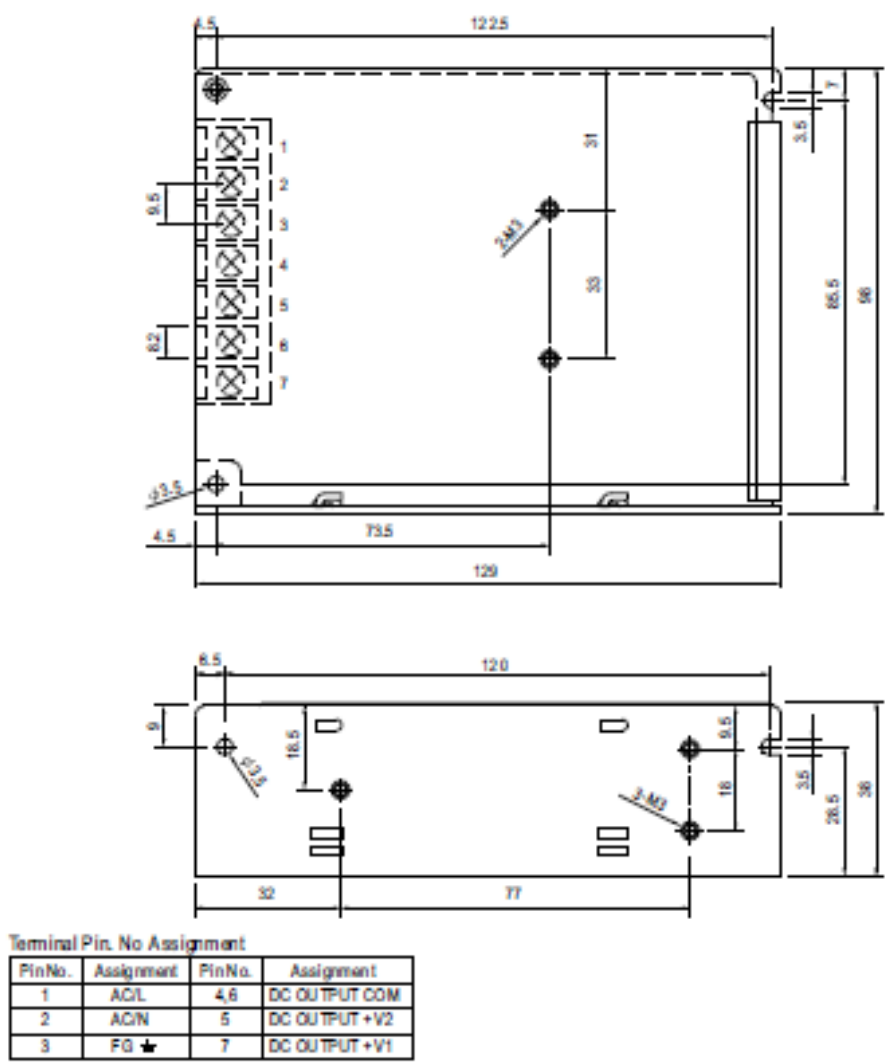

\section{Derating Curve}

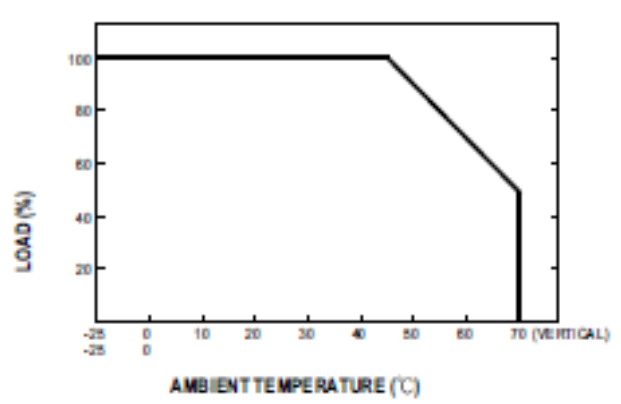

- Output Derafing VS Input Voltage

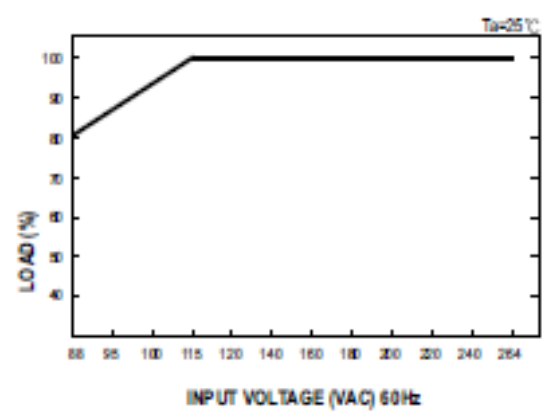

Tel: 1-973-779-8282

www.trcelectronics.com

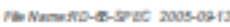




\section{Appendix D: Control Logic \& Programming}
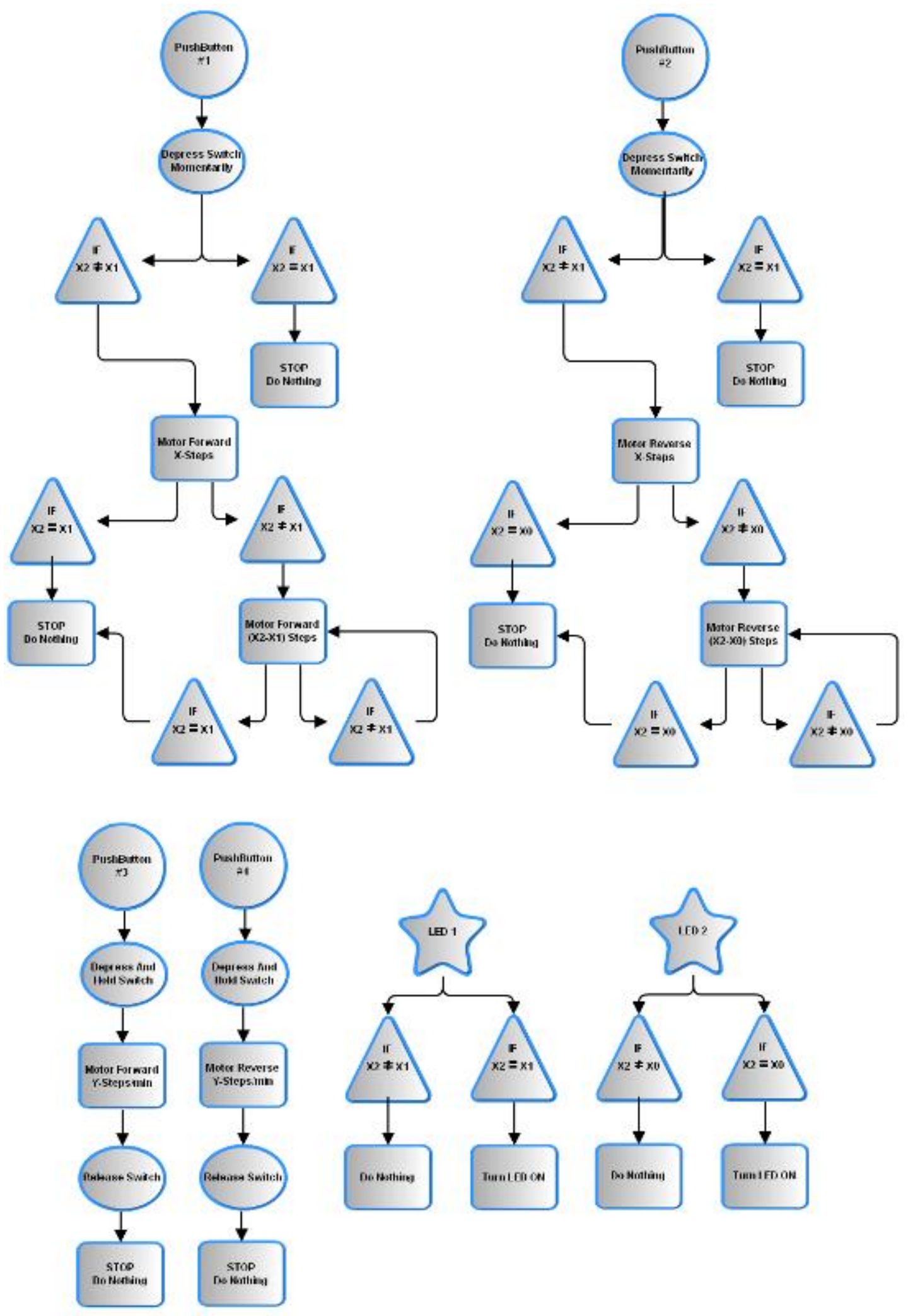


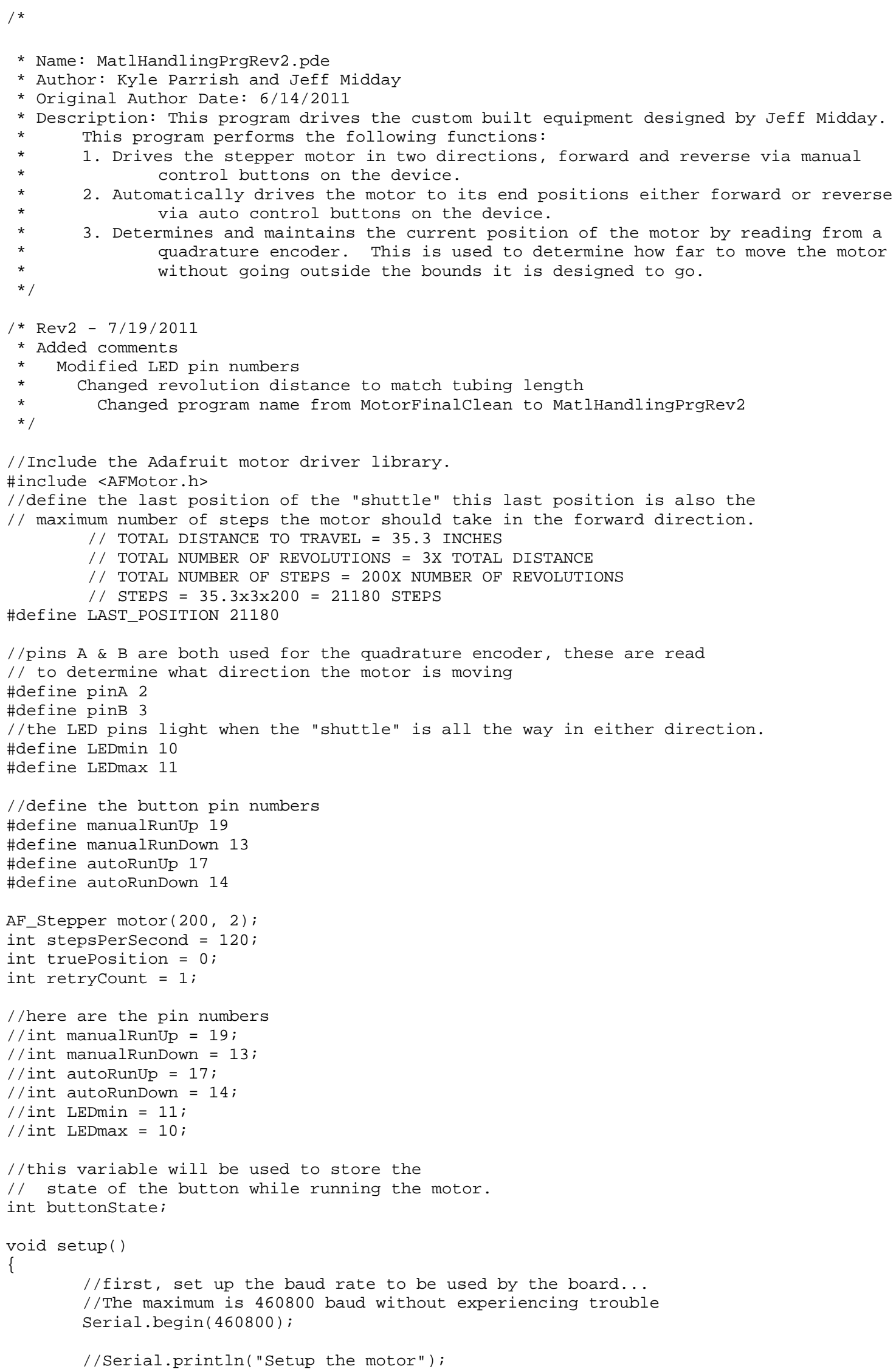




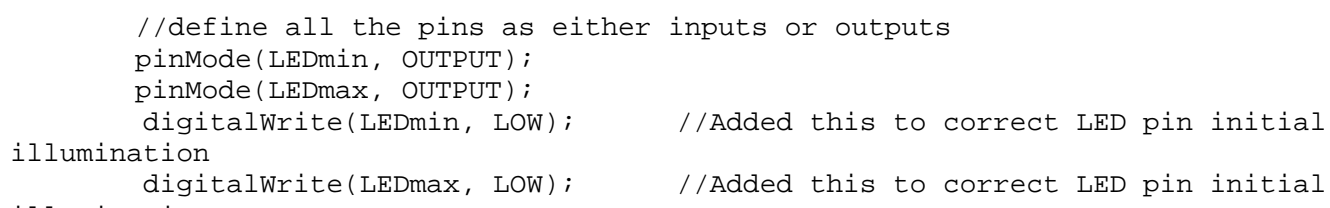
illumination

pinMode(manualRunUp, INPUT); pinMode (manualRunDown, INPUT); pinMode(autoRunUp, INPUT); pinMode(autoRunDown, INPUT);

pinMode(pinA, INPUT);

digitalWrite(pinA, HIGH); //I'm not sure why this is needed but was highly recommended in the

//documentation that I read,

something about clearing residual charge...

pinMode(pinB, INPUT);

digitalWrite(pinB, HIGH);

//attach an interrupt in slot $\odot$ to the function doEncoder, and then do it only on the RISING change,

// when we ran this on any change, the number that was generated out of the encoder was double what

// it really was, this might be because CHANGE is counting microsteps. attachInterrupt $(\odot$, doEncoder, RISING);

//set the motor speed, for our current setup we decided that 120 RPM is the fastet that we can run.

$(16 \mathrm{MHz})$

//limited by the maximum baud rate and clock speed of the board

\}

motor.setSpeed(120); //120

void loop( )

\{

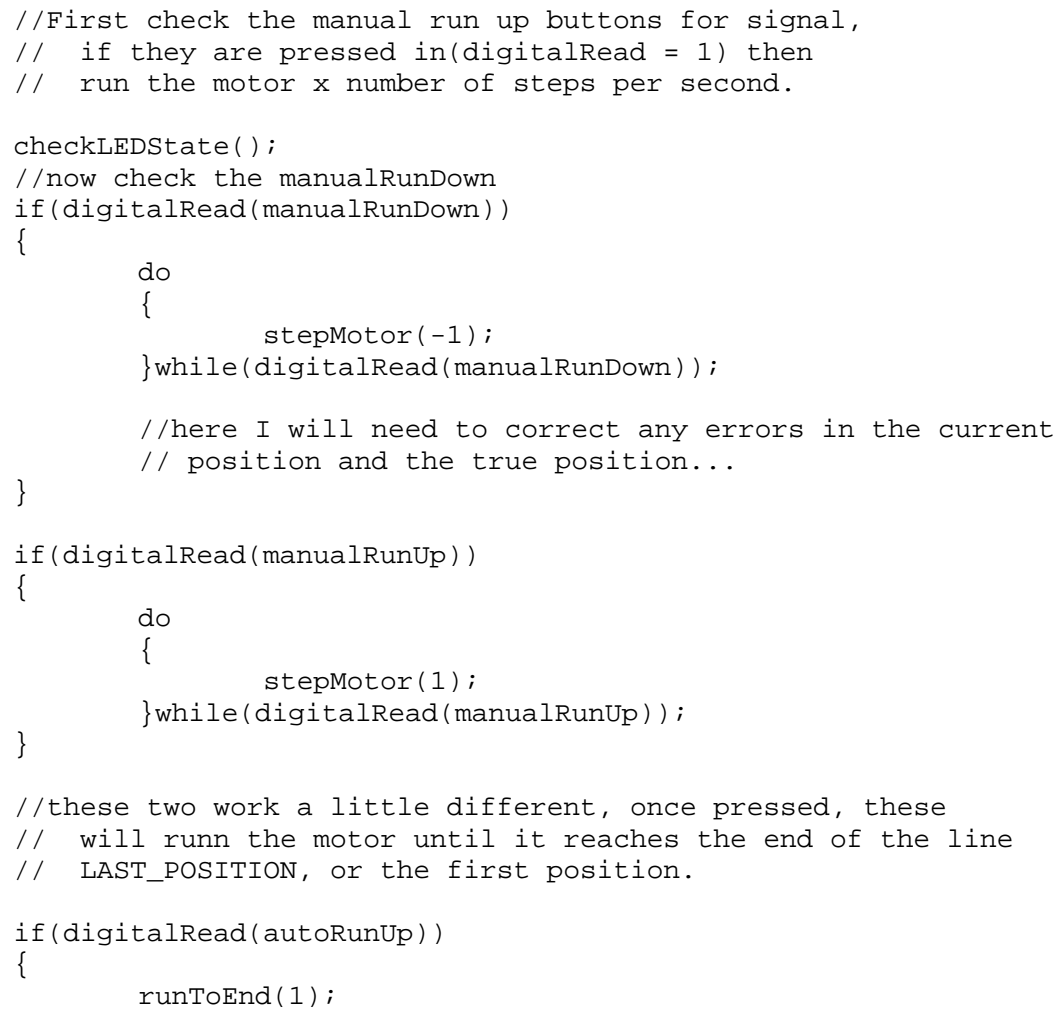




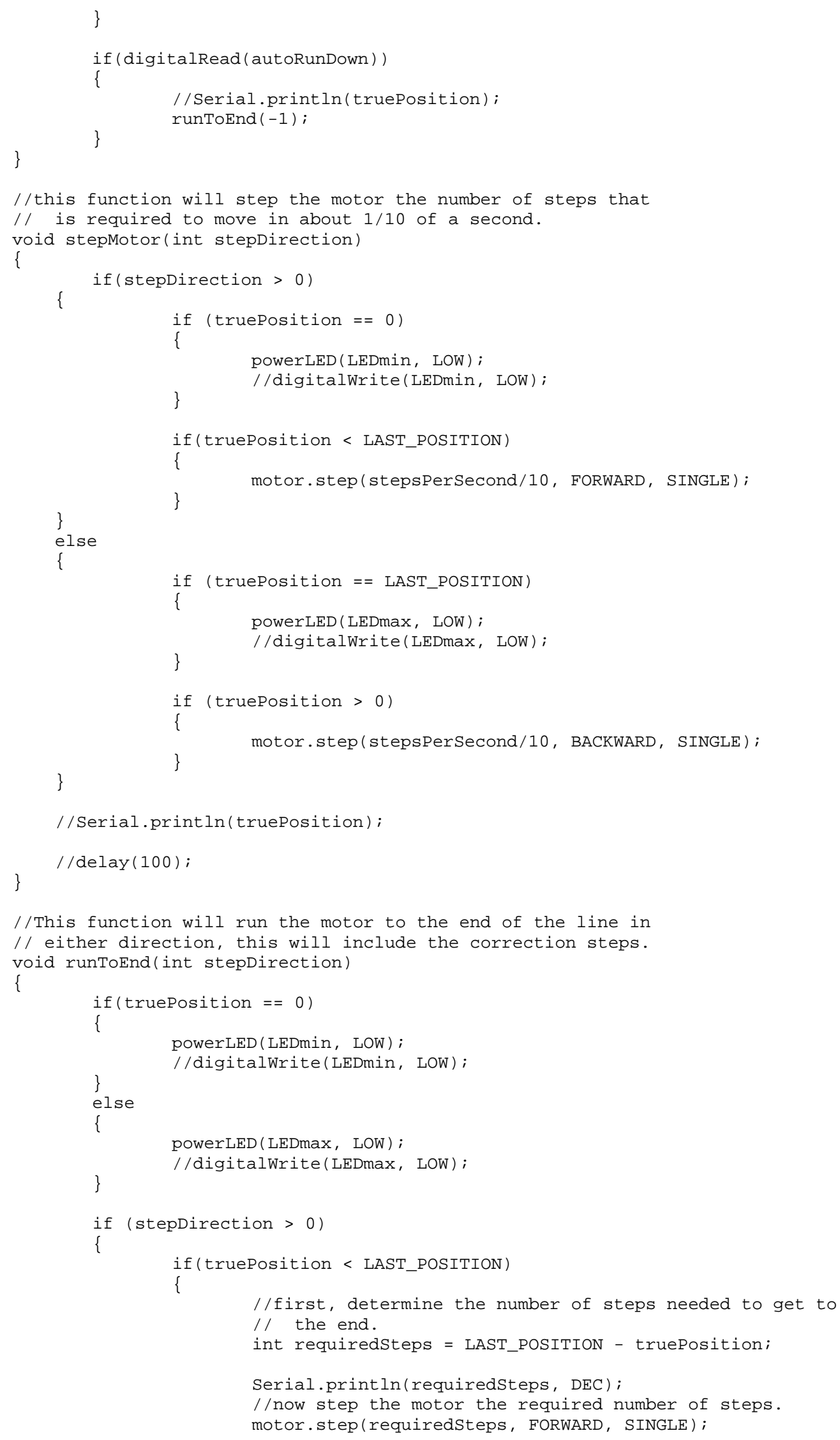


//Serial.println(truePosition);

//Now check to make sure the shuttle is in the correct // position.

retrycount $=1$;

/*

error checking later on but now

this code is not currently in use, it may be needed to do while(truePosition <= LAST_POSITION || retrycount < 4)

\{

//they do not match, adjust accordingly...

int positionDifference = LAST_POSITION - truePosition;

//if the difference is negative, then the true position

// thinks that it is past the last position, we will not

// move the shuttle in this case because this is an

// unexpected instance that should be fixed with the

// manual movement.

if (positionDifference $>0$ )

\{

$/ /$ now move the motor the difference //motor.step(positionDifference, FORWARD, SINGLE); retrycount++;

else

\{

\}

break;

\}

\}

\}

else

\{

//we are moving back to the zero position, we just need

// to move the shuttle the true position backwards.

if (truePosition $>0$ )

\{

motor.step(truePosition, BACKWARD, SINGLE);

if (truePosition $>0$ )

\{

//move the motor again...

\}

//motor.step(truePosition, BACKWARD, SINGLE);

\}

//This function checks to see what, if any LEDs need to be on void checkLEDState( )

\{

//Since only once LED can be on at any given point, this logic can be combined

// a single if-the-else statement

$/ /$ first check if it is at zero

if $($ truePosition $==0$ )

\{

//it is at zero, light the zero LED...

powerLED(LEDmin, HIGH);

\}

//digitalWrite(LEDmin, HIGH);

else if(truePosition == LAST_POSITION)

\{

//it is in the last position, light the endpoint LED...

powerLED(LEDmax, HIGH);

//digitalWrite(LEDmax, HIGH); 


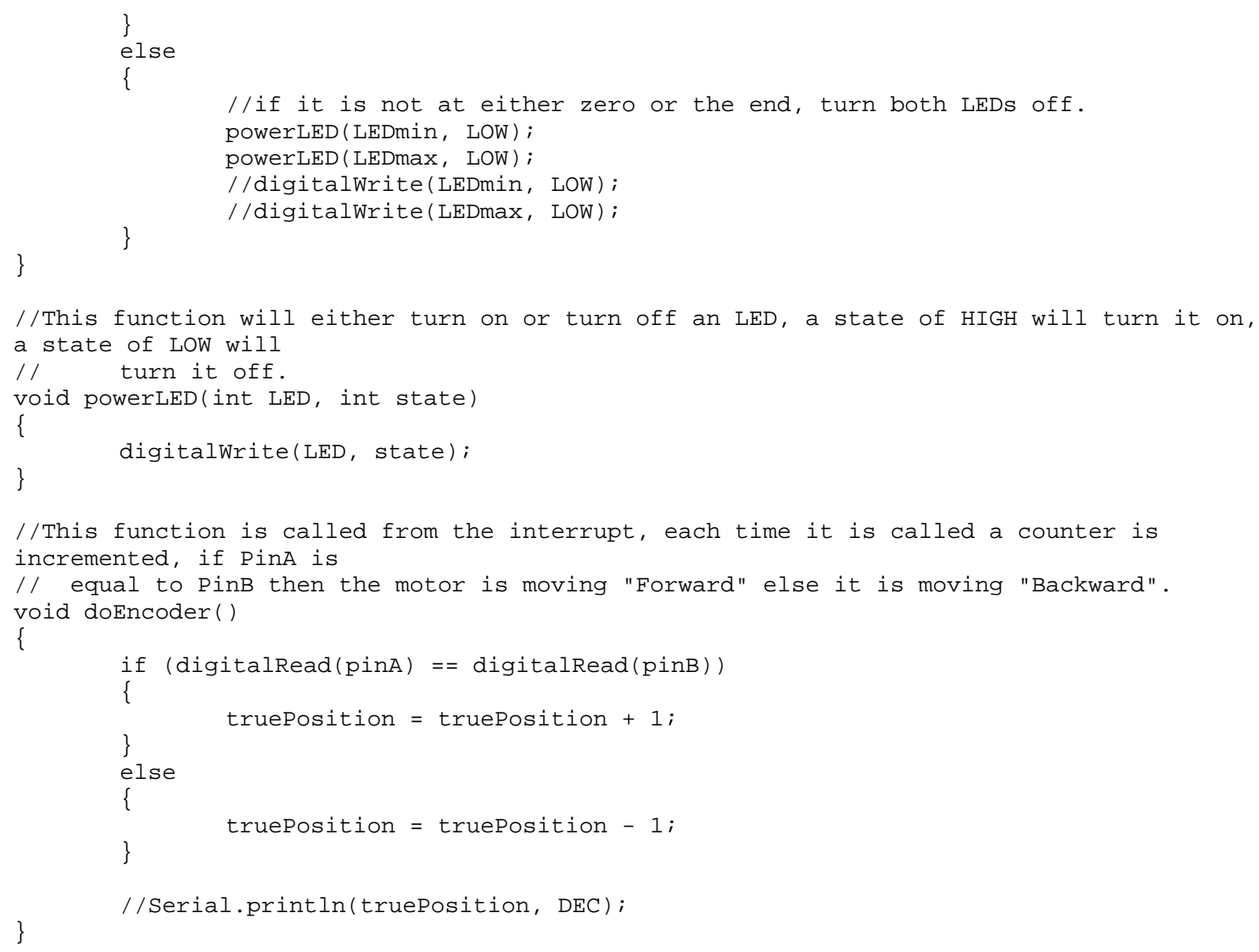




\section{Appendix E: LS-Dyna - Deck Code}

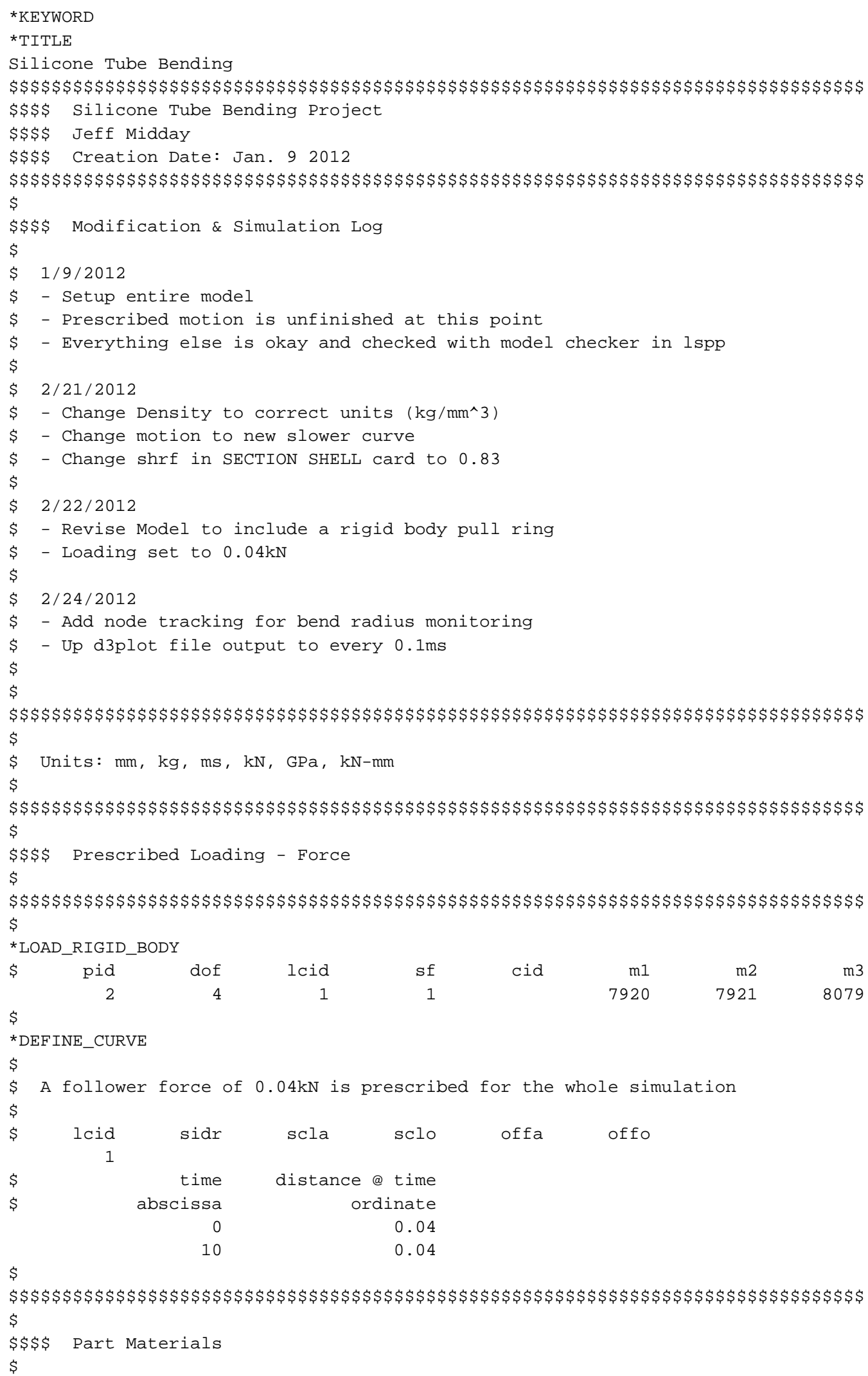




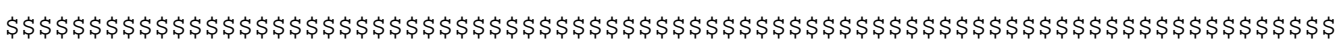

$\$$

\$ Use Blatz-Ko Rubber

$\$$ poisson's ratio $=0.463$ (default for material card)

$\$$ density $=1210 \mathrm{~kg} / \mathrm{m}^{\wedge} 3=1.21 \mathrm{E}-6 \mathrm{~kg} / \mathrm{mm} \wedge 3$

$\$$ shear modulus $=3.6 \mathrm{MPa}=0.0036 \mathrm{GPa}$

$\$$

*MAT_BLATZ-KO_RUBBER

$\begin{array}{rrrr}\$ & \mathrm{R} \odot & \mathrm{G} & \mathrm{REF} \\ 1 & 1.21 \mathrm{E}-6 & 0.0036 & \end{array}$

$\$$ The properties of the rigid pull ring do not really matter.

$\$$

*MAT_RIGID

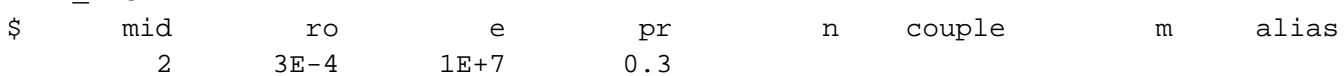

$\$$

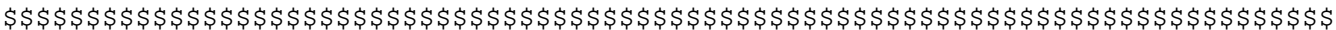

$\$$

$\$ \$ \$$ Element Section

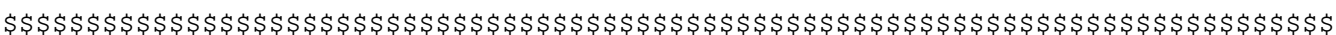

$\$$

*SECTION_SHELL

$\$$ Use default Belytschko-Tsay formulation

Shell Thickness (2.0 mm thick)

\$ sid elform shrf nip propt qr/irid icomp

$\$$

1

20.83

t3

3. 0

2.0

2.0

2.0

2.0

nloc

$\$$

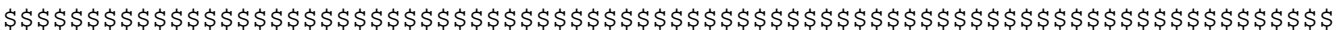

$\$ \$ \$$ Controls

$\$$

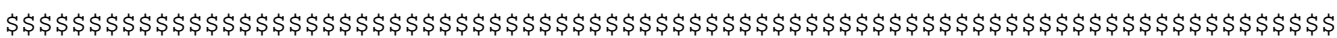

$\$$

${ }^{*}$ CONTROL_TIMESTEP

$\$$ dtinit tssfac isdo tslimt dt2ms lctm erode ms1st

$\$$

*CONTROL_tERMINATION

$\$$ endtim endcyc dtmin endeng endmas

10.0

$\$$

*CONTROL_ENERGY

\$ hgen rwen slnten rylen

$\$$

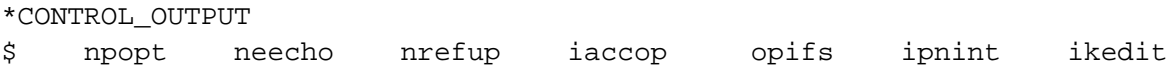

$\$$

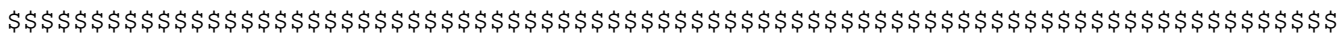

$\$$

$\$ \$$ Database Files

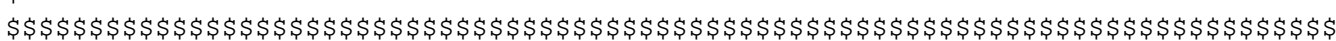


$\$$

*DATABASE_BINARY_D3PLOT

$\$ d t \quad l c d t$

0.1

$\$$

*DATABASE_EXTENT_BINARY

$\$$ neiph neips maxint strflg sigflg epsflg rltflg engflg

\$ cmpflg ieverp beamip dcomp shge stssz n3thdt

$\$$

*DATABASE_GLSTAT

$\$ d t$

0.05

$\$$

*DATABASE_MATSUM

$\$ d t$

0.05

*DATABASE_ELOUT

$\$ d t$

0.05

$\$$

*DATABASE_RCFORC

$\$ \quad \mathrm{dt}$

0.05

$\$$

*DATABASE_SLEOUT

$\$ \quad \mathrm{dt}$

0.05

*DATABASE_NODOUT

$\$ d t$

0.05

$\$$

*DATABASE_HISTORY_NODE

$\$$

node location

id1 - at left of bent section

id2 - at right of bent section

id3 - at top of bent section

id4 - at bottom of bent section

All others - on the Left Side View for bend-radius tracking ( $X \& Z$ coords)

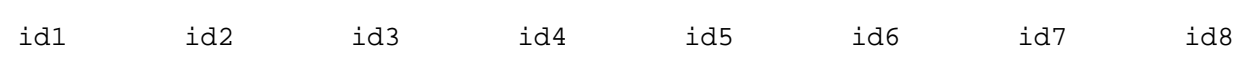

$\begin{array}{llllllll}1502 & 1541 & 1522 & 1561 & 1 & 791 & 1581 & 2371\end{array}$

$\begin{array}{lllllll}3161 & 3951 & 4741 & 5531 & 6321 & 7111 & 7901\end{array}$

$\$$

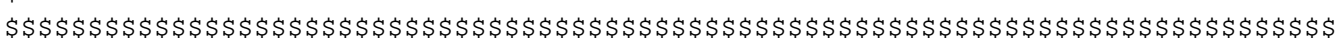

$\$$

$\$ \$ \$$ SPC Constraints

$\$$

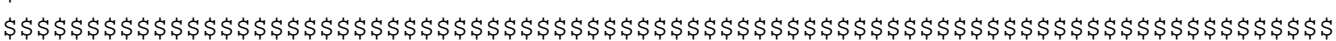

$\$$

$\$$ Zero dof to all nodes in set - fixed in space

$\$$

*BOUNDARY_SPC_SET

$\$$

nsid

1

cid dofx

1 dofy

1

dofz

dofrx

dofry

dofrz

$\begin{array}{lllll}1 & 1 & 1 & 1 & 1\end{array}$




\begin{tabular}{|c|c|c|c|c|c|c|c|c|}
\hline \multicolumn{9}{|c|}{ 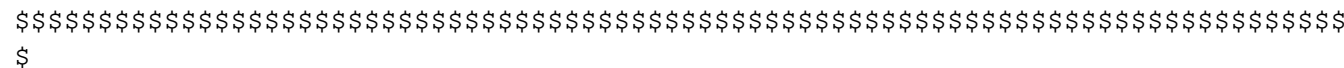 } \\
\hline \multicolumn{9}{|c|}{$\begin{array}{l}\$ \$ \$ \text { Node Sets, Part Cards, Elements \& Nodes } \\
\$\end{array}$} \\
\hline \multicolumn{9}{|c|}{ 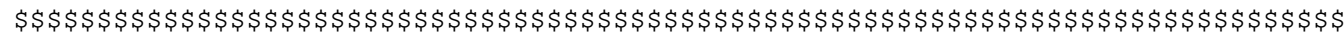 } \\
\hline \multicolumn{9}{|c|}{ *PART } \\
\hline \multicolumn{9}{|c|}{ Silicone_Tube } \\
\hline \multirow[t]{2}{*}{$\$$} & pid & secid & $\operatorname{mid}$ & eosid & hgid & grav & adpopt & tmid \\
\hline & 1 & 1 & 1 & 0 & 0 & 0 & 0 & 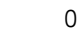 \\
\hline \multicolumn{9}{|l|}{$\$$} \\
\hline \multicolumn{9}{|c|}{ Rigid_Pull_Ring } \\
\hline \multirow[t]{2}{*}{ \$ } & pid & secid & mid & eosid & hgid & grav & adpopt & tmid \\
\hline & 2 & 1 & 2 & $\odot$ & 0 & 0 & $\odot$ & $\theta$ \\
\hline \multicolumn{9}{|l|}{ \$ } \\
\hline \multicolumn{9}{|c|}{ *SET_NODE_LIST_TITLE } \\
\hline \multirow[t]{2}{*}{$\$$} & sid & da1 & da2 & da3 & da4 & solver & & \\
\hline & 1 & 0.000 & 0.000 & 0.000 & 0.000 & & & \\
\hline \multirow[t]{11}{*}{$\$$} & nid1 & nid2 & nid3 & nid4 & nid5 & nid6 & nid7 & nid8 \\
\hline & 1 & 2 & 3 & 4 & 5 & 6 & 7 & 8 \\
\hline & 9 & 10 & 11 & 12 & 13 & 14 & 15 & 16 \\
\hline & 17 & 18 & 19 & 20 & 21 & 22 & 23 & 24 \\
\hline & 25 & 26 & 27 & 28 & 29 & 30 & 31 & 32 \\
\hline & 33 & 34 & 35 & 36 & 37 & 38 & 39 & 40 \\
\hline & 41 & 42 & 43 & 44 & 45 & 46 & 47 & 48 \\
\hline & 49 & 50 & 51 & 52 & 53 & 54 & 55 & 56 \\
\hline & 57 & 58 & 59 & 60 & 61 & 62 & 63 & 64 \\
\hline & 65 & 66 & 67 & 68 & 69 & 70 & 71 & 72 \\
\hline & 73 & 74 & 75 & 76 & 77 & 78 & 79 & $\odot$ \\
\hline
\end{tabular}

$\$$ 


\section{Appendix F: Matlab FEA Processing Code}

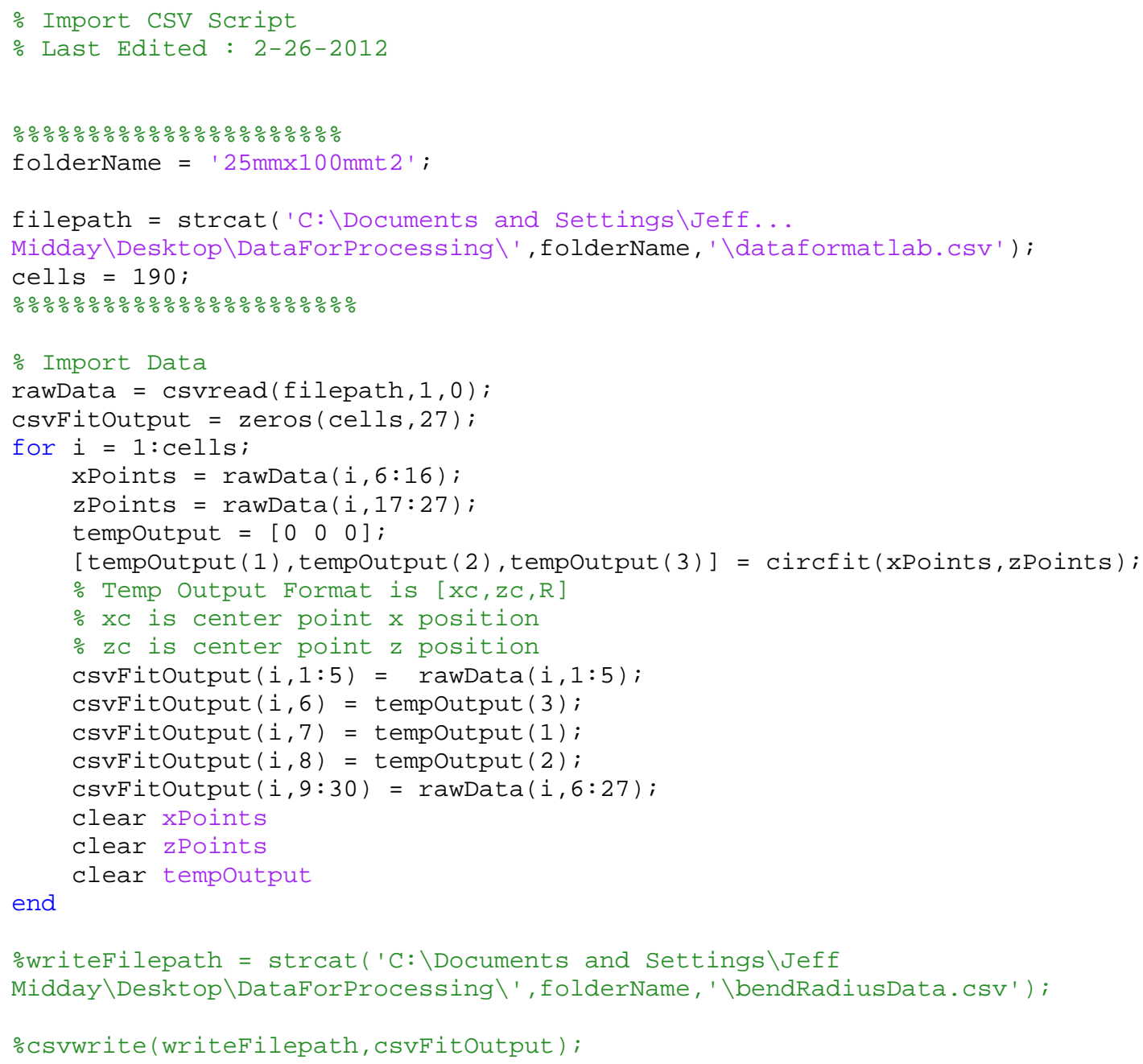




\section{Appendix G: Bill of Materials}

\begin{tabular}{|c|c|c|c|c|c|c|c|c|}
\hline \multicolumn{9}{|c|}{ Bill of Materials } \\
\hline Item\# & Part \# & Part Name & Description & Manufacturer & Quantity & Unit & Unit Cost & Total Cost \\
\hline \multicolumn{9}{|c|}{1 - Insertion Assembly } \\
\hline 1.1 & - & Silicone_Tube & Multi-Lumen Extruded Silicone Tubing 80 Durometer, .625" ID & Specialty Silicone Fabricators & & Pc. & $\$ 6,450.00$ & $\$ 6,450.00$ \\
\hline 1.2 & $5054 \mathrm{~K} 25$ & Drive_Spring_Rev2 & Ø0.585 x 42" Custom Compression Spring - Stainless Steel & Marshall Spring & 1 & Pc. & $\$ 140.00$ & $\$ 140.00$ \\
\hline 1.3 & CLM-3-SBP-1 & Spring Plungers & $3 \mathrm{~mm}$ Ball Spring Plungers & Carr Lane & 2 & Pc. & $\$ 6.95$ & $\$ 13.90$ \\
\hline 1.4 & Q\#: 2031511-2 & Shuttle_Rev3 & Line Item \#2: SLS Standard NyTek 1200 CF & Solid Concepts & 1 & Pc. & $\$ 83.00$ & $\$ 83.00$ \\
\hline 1.5 & 17Y402D-LW4-200SI & Stepper_Motor & 1.8 ${ }^{\circ}$ Step Motor. $100 \mathrm{oz}$-in torque w/ SingleEnd Indexed 200 step encoder & Anaheim Automation & 1 & Pc. & $\$ 108.33$ & $\$ 108.33$ \\
\hline 1.6 & Q\#: 2011792-2 & Motor_Coupling_RP_Rev2 & SLS NyT ek $1200 \mathrm{CFw} /$ three threaded inserts & Solid Concepts & 1 & Pc. & $\$ 83.00$ & $\$ 83.00$ \\
\hline 1.7 & Q\#: S11-068 & \begin{tabular}{|l|} 
Tube Coating \\
\end{tabular} & SlickSil Coating on 25 multi-lumen Silicone tubes. & Surface Solutions Group LLC & 1 & Pc. & $\$ 20.00$ & $\$ 20.00$ \\
\hline 1.8 & $9452 \mathrm{~K} 72$ & O-Ring & \#018 Buna-N O-Ring, 100ct & McMaster Carr & 1 & Pack & $\$ 3.33$ & $\$ 3.33$ \\
\hline 1.9 & $1243 \mathrm{~K} 31$ & Grease & High Temp PTFE Grease $0.750 z$ & McMaster Carr & 1 & Tube & $\$ 4.87$ & $\$ 4.87$ \\
\hline 1.10 & Q\#: 2045474-1 & Nose_Cap_POINT_SOFT & Nose_Cap_POINT_SOFT_Rev1 Tango Plus PolyJet & Solid Concepts & 1 & Pc. & $\$ 69.00$ & $\$ 69.00$ \\
\hline 1.11 & N35B.100.500.250 & Magnets & Nickel Plated Neo Magnet 1"x.5"x.25" & Magnets Online & 4 & Pc. & $\$ 4.50$ & $\$ 18.00$ \\
\hline 1.12 & Q\#: 2047512-1 & Proximal_Coupler_Modified & Item \#2: NyTek 1200CF Proximal_Coupler_Modified & Solid Concepts & 1 & Pc. & $\$ 116.00$ & $\$ 116.00$ \\
\hline 1.13 & Q\#: 2047512-1 & Spring Coupling & Item \#3: NyTek 1200CF Spring_Coupling_Rev3 & Solid Concepts & 1 & Pc. & $\$ 87.00$ & $\$ 87.00$ \\
\hline 1.14 & Q\#: 2047512-2 & Nose Cap & Item \#1: DMLS 14-7 SS Shotpeen Nose_Cap_Modified_Rev2 & Solid Concepts & 1 & Pc. & $\$ 132.00$ & $\$ 132.00$ \\
\hline & & & & & & & Total & $\$ 7,328.43$ \\
\hline
\end{tabular}




\begin{tabular}{|c|c|c|c|c|c|c|c|c|}
\hline \multicolumn{9}{|c|}{ Bill of Materials } \\
\hline Item\# & Part \# & Part Name & Description & Manufacturer & Quantity & Unit & Unit Cost & Total Cost \\
\hline \multicolumn{9}{|c|}{2 - Other Features } \\
\hline 2.1 & SLIPVCC37 & Camera Coupler & $37 \mathrm{~mm}$ threaded camera coupler & The Tool Warehouse & 1 & Pc. & $\$ 28.95$ & $\$ 28.95$ \\
\hline 2.2 & - & Fiberscope & 48" 6mm Rubber Hose Fiber Optic Scope & Anaconda Universal Products Inc. & 1 & Pc. & $\$ 300.00$ & $\$ 300.00$ \\
\hline 2.3 & $8701 \mathrm{~K} 861$ & Bushing & 1.125" UHMWP Plastic Rod & McMaster Carr & 3 & Feet & $\$ 2.52$ & $\$ 7.56$ \\
\hline 2.4 & 2418T117 & O-Ring & Soft Buna-N O-Ring 5/16"ID, 1/8" Wall & McMaster Carr & 1 & Pack & $\$ 9.97$ & $\$ 9.97$ \\
\hline 2.5 & 7129 T33 & Knob & 1/4-20 Knob w/ 1/2" Shaft & McMaster Carr & 6 & Pc. & $\$ 1.84$ & $\$ 11.04$ \\
\hline 2.6 & 5407K65 & Clamp & 1.25"-2.25" Worm Band Clamp & McMaster Carr & 1 & Pack & $\$ 11.38$ & $\$ 11.38$ \\
\hline 2.7 & - & Stand & 3 Way LCD Monitor TV Desk Mount Adjustable Tiliting Stand & Ebay & 1 & Pc. & $\$ 34.22$ & $\$ 34.22$ \\
\hline 2.8 & - & Lens Adapter & 67mm Tube Adapter for Nikon Coolpix L120 Lens & Amazon & 1 & Pc. & $\$ 19.99$ & $\$ 19.99$ \\
\hline 2.9 & - & Lens Adapter & Adorama Step-Down adapter ring $67 \mathrm{~mm}$ lens to $52 \mathrm{~mm}$ filter & Amazon & 1 & Pc. & $\$ 3.44$ & $\$ 3.44$ \\
\hline 2.10 & - & Memory Card & Transcend 16GB Class 10 SDHC Memory Card (TS16GSDHC10) & Amazon & 1 & Pc. & $\$ 24.21$ & $\$ 24.21$ \\
\hline 2.11 & - & Carry Case & Zeikos ZE-CA48B Deluxe Soft Medium Camera and Video Bag & Amazon & 1 & Pc. & $\$ 7.18$ & $\$ 7.18$ \\
\hline 2.12 & NIKCPL120RD & Nikon Camera & Nikon Coolpix L120 Red 14.1MP Digital Camera (Nikon \#26254) & Tri State Camera \& Video & 1 & Pc. & $\$ 249.00$ & $\$ 249.00$ \\
\hline 2.13 & Q\#: 0012461 & Camera_Mount & Camera_Mount Fabrication \& Material & TMCO & 1 & Pc. & $\$ 57.18$ & $\$ 57.18$ \\
\hline 2.14 & Q\#: 2060926-1 & Trocar Port & Port.STL in NyTek 1200CF Mat'l & Solid Concepts & 1 & Pc. & $\$ 101.00$ & $\$ 101.00$ \\
\hline 2.15 & - & Trocar Tube & Drawn Aluminum Bare Tube $6061 \mathrm{~T} 6$ & Online Metals & 1 & Foot & $\$ 5.82$ & $\$ 5.82$ \\
\hline 2.16 & - & Trocar Punch & Cold Finish Aluminum Round 6061 T651 & Online Metals & 1 & Foot & $\$ 10.07$ & $\$ 10.07$ \\
\hline 2.17 & $9452 \mathrm{~K} 84$ & O-Ring & Bbuna-N O-Ring AS568A Dash\# 120, 100 pack & McMaster Carr & 1 & Pack & $\$ 4.52$ & $\$ 4.52$ \\
\hline 2.18 & CL-4-CP & Trocar Handle & 1/2 Clamping Pin @ 5 inches long & Carr Lane & 1 & Pc. & $\$ 8.25$ & $\$ 8.25$ \\
\hline & & & & & & & Total & $\$ 893.78$ \\
\hline
\end{tabular}




\begin{tabular}{|c|c|c|c|c|c|c|c|c|}
\hline \multicolumn{9}{|c|}{ Bill of Materials } \\
\hline Item\# & Part \# & Part Name & Description & Manufacturer & Quantity & Unit & Unit Cost & Total Cost \\
\hline \multicolumn{9}{|c|}{3 - Control System } \\
\hline 3.1 & $532-501200 \mathrm{~B} 00$ & Heat Sink & DIP 16 Finned Heat Sink for Motor Shield & Mouser & 3 & Pc. & $\$ 0.59$ & $\$ 1.77$ \\
\hline 3.2 & 511-L293B & L293B & L293B Chip (Increases Motor Shield Current Capacity for Arduino) & Mouser & 2 & Pc. & $\$ 3.89$ & $\$ 7.78$ \\
\hline 3.3 & RB-Ada-02 & Motor Shield & Motor Shield Kit for Arduino (Drive 2 Steppers, $1.2 \mathrm{~mA}$ max & Robot Shop & 1 & Pc. & $\$ 19.50$ & $\$ 19.50$ \\
\hline 3.4 & RB-Phi-36 & USB Cable & 6' USB A to B Cable (For Arduino Communication) & Robot Shop & 1 & Pc. & $\$ 2.84$ & $\$ 2.84$ \\
\hline 3.5 & RB-Spa-375 & Barrel Jack & 9V to 5.5 x 2.1mm Barrel Jack (For Arduino Power to PC Power Supply) & Robot Shop & 1 & Pc. & $\$ 2.95$ & $\$ 2.95$ \\
\hline 3.6 & RB-lbo-84 & Wire & \#22 Gauge Hook-Up Wire 25' & Robot Shop & 1 & Pc. & $\$ 2.25$ & $\$ 2.25$ \\
\hline 3.7 & RB-Ada-08 & Headers & Motor Shield Stacking Headers & Robot Shop & 4 & Pc. & $\$ 2.00$ & $\$ 8.00$ \\
\hline 3.8 & 92185A106 & Fastener & 316 SS, 4-40 x 1/4" SHCS, pack of 50 & McMaster Carr & 1 & Pack & $\$ 4.93$ & $\$ 4.93$ \\
\hline 3.9 & $7665 \mathrm{~K} 11$ & Rubber Gasket & $1 / 2$ " x 1/16" x 36" Rubber Gasket & McMaster Carr & 2 & Pc. & $\$ 2.25$ & $\$ 4.50$ \\
\hline 3.10 & $8507 \mathrm{~K} 52$ & Silicone Gasket & Silicone Rubber Edging for 1/16" Plate & McMaster Carr & 10 & Feet & $\$ 0.77$ & $\$ 7.70$ \\
\hline 3.11 & 91251A197 & Fastener & Black Oxide Steel 8-32 x 3/4" SHCS, pack of 100 & McMaster Carr & 1 & Pack & $\$ 10.84$ & $\$ 10.84$ \\
\hline 3.12 & 91292A114 & Fastener & $18-8$ SS M3 x $12 \mathrm{~mm}$ SHCS, pack of 100 & McMaster Carr & 1 & Pack & $\$ 3.15$ & $\$ 3.15$ \\
\hline 3.13 & 9013K811 & Rubber Sheet & Rubber Sheet 6"'x6"x1/16" Neoprene & McMaster Carr & 1 & Pc. & $\$ 4.20$ & $\$ 4.20$ \\
\hline 3.14 & 92196A108 & Fastener & 18-8 SS, 4-40 x 3/8" SHCS, pack of 100 & McMaster Carr & 1 & Pack & $\$ 2.87$ & $\$ 2.87$ \\
\hline 3.15 & RD-65B & Power Supply & AC/DC 68W Switching Power Supply 5V/8A, 24V/3A, 110VAC input & TRC Electronics & 1 & Pc. & $\$ 36.43$ & $\$ 36.43$ \\
\hline 3.16 & 611-AP4E202T ZBE & Push Button & Pushbutton Switch BiColor LED (Red/Green) 5V input (24V Max) & Mouser & 2 & Pc. & $\$ 15.30$ & $\$ 30.60$ \\
\hline 3.17 & 611-AP4D207TZBE & Push Button & Pushbutton Switch SuperBlue LED 5V input (24V Max) & Mouser & 2 & Pc. & $\$ 18.87$ & $\$ 37.74$ \\
\hline 3.18 & 526-HS-ASST-9 & Heat Shrink & NTE Heat Shrink Kit & Mouser & 1 & Pc. & $\$ 14.33$ & $\$ 14.33$ \\
\hline 3.19 & $845-5507560$ & Cable Gland & Altech Cable Gland M12x1.5 & Mouser & 2 & Pc. & $\$ 2.90$ & $\$ 5.80$ \\
\hline 3.20 & 607-5102H5-5V & LED & Chicago Miniature Ø1/4" Green Panel Mount LED & Mouser & 4 & Pc. & $\$ 1.66$ & $\$ 6.64$ \\
\hline 3.21 & $538-88732-9002$ & USB Cable & Molex USB A to B Cable 2.69' & Mouser & 1 & Pc. & $\$ 2.27$ & $\$ 2.27$ \\
\hline 3.22 & 706-17-200321 & USB Connector & Conec USB A type F/F Panel Connector & Mouser & 1 & Pc. & $\$ 18.25$ & $\$ 18.25$ \\
\hline 3.23 & \begin{tabular}{|c|}
- \\
\end{tabular} & Push_Button_Panel_Rev1 & Stainless Steel Push Button Panel & TMCO & 1 & Pc. & $\$ 90.00$ & $\$ 90.00$ \\
\hline 3.24 & 5444 & USB Cable & USB Male-A to Male-A $10 \mathrm{ft}$. & MonoPrice & 1 & Pc. & $\$ 1.50$ & $\$ 1.50$ \\
\hline 3.25 & 5280 & Power Cable & 10ft. 18AWG Power Cord Cable & MonoPrice & 1 & Pc. & $\$ 1.97$ & $\$ 1.97$ \\
\hline 3.26 & 92319A653 & Standoff & 5/8" long, 4-40 Circuitboard Standoff Nylon 6/6 & McMaster Carr & 8 & Pc. & $\$ 1.26$ & $\$ 10.08$ \\
\hline 3.27 & AM010 & Microcontroller & Ruggeduino 24V Microcontroller & Rugged Circuits & 1 & Pc. & $\$ 39.95$ & $\$ 39.95$ \\
\hline 3.28 & 1053-1228-ND & Fan & 5V Square DC Fan 60mmx15mm 4500rpm 19CFM Fan (Orion Fans) & DigiKey & 2 & Pc. & $\$ 10.26$ & $\$ 20.52$ \\
\hline 3.29 & 1053-1378-ND & Fan Guard & Metal Fan Guard (Orion Fans) & DigiKey & 2 & Pc. & $\$ 1.37$ & $\$ 2.74$ \\
\hline 3.30 & N35B.100.500.100 & Magnets & 1" X .5" X .1" Grade 35 Neo Block Nickel Plated & Magnets Online & 2 & Pc. & $\$ 6.00$ & $\$ 12.00$ \\
\hline 3.31 & - & Base Plate & Fabrication \& Material for Base Plate Part & UNL Machine Shop & & Pc. & $\$ 218.00$ & $\$ 218.00$ \\
\hline & & & & & & & Total & $\$ 632.10$ \\
\hline
\end{tabular}




\section{Appendix H: Assembly Drawings}

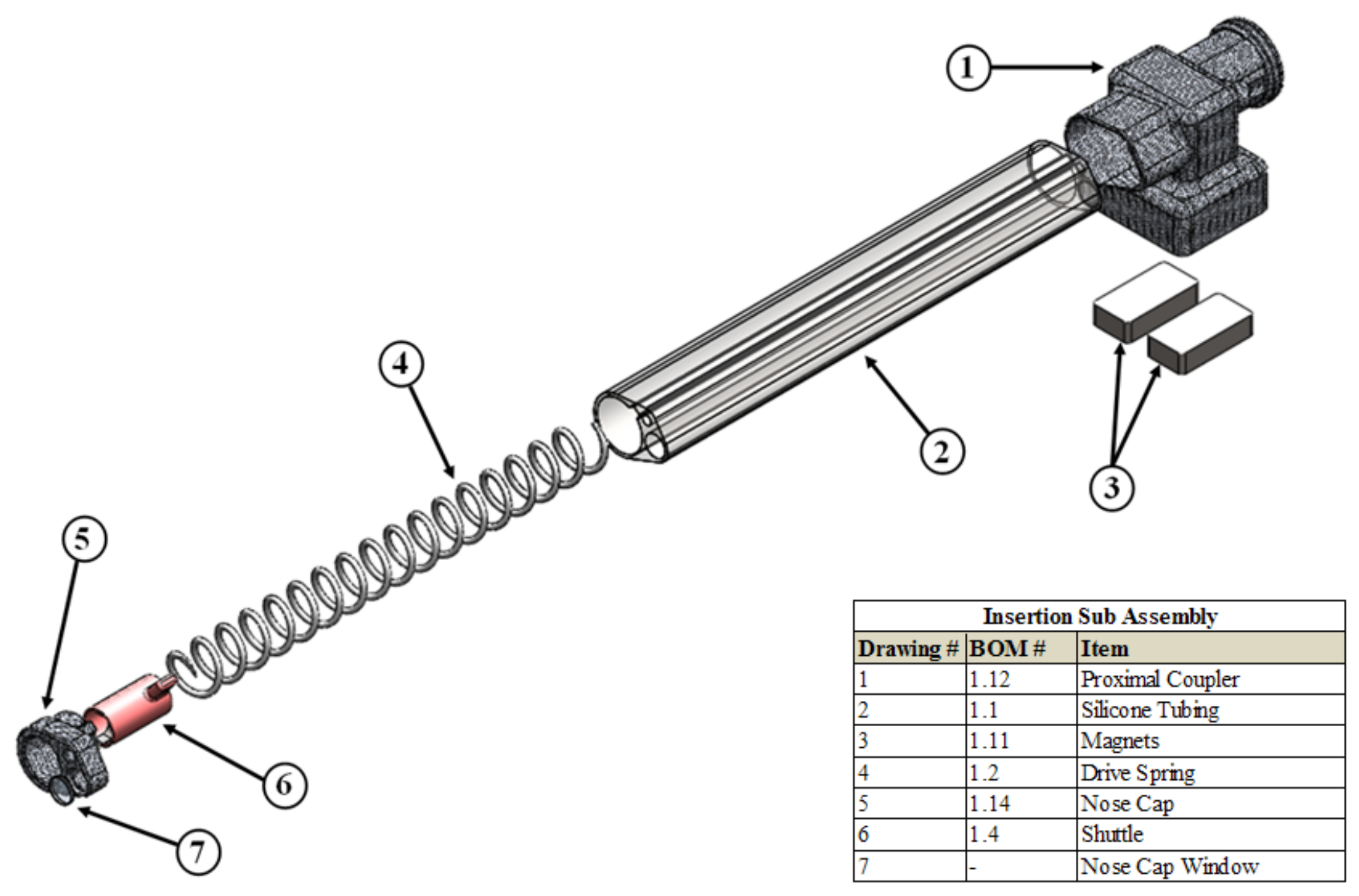




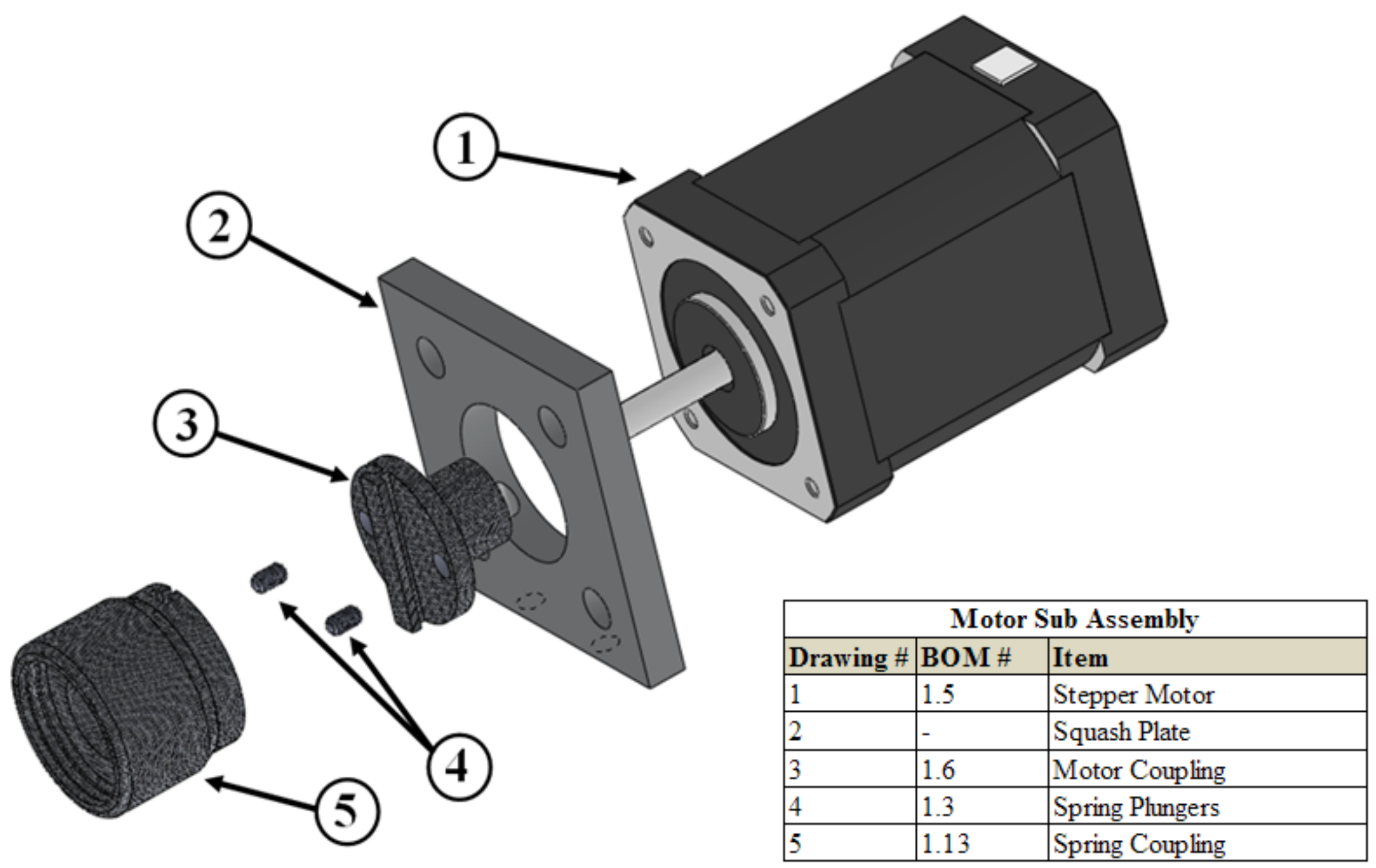




\begin{tabular}{|l|l|l|}
\hline \multicolumn{3}{|c|}{ Complete Assembly } \\
\hline Drawing \# & BOM \# & Item \\
\hline 1 & $3.16 / 3.17$ & Push Buttons \\
\hline 2 & 3.20 & LED Power Indicator \\
\hline 3 & 3.23 & Push Button Panel \\
\hline 4 & 3.22 & USB Connector \\
\hline 5 & 3.20 & LED Position Indicators \\
\hline 6 & 3.15 & Power Supply \\
\hline 7 & 3.27 & Ruggeduino \\
\hline 8 & 3.31 & Base Plate \\
\hline 9 & - & Heat Sink \\
\hline 10 & - & Insertion Sub Assembly \\
\hline 11 & 3.30 & Magnets \\
\hline 12 & - & Motor Sub Assembly \\
\hline 13 & 2.2 & Fiberscope \\
\hline 14 & 2.13 & Camera Mount \\
\hline 15 & 2.1 & Camera Coupler \\
\hline 16 & 2.12 & Camera \\
\hline
\end{tabular}

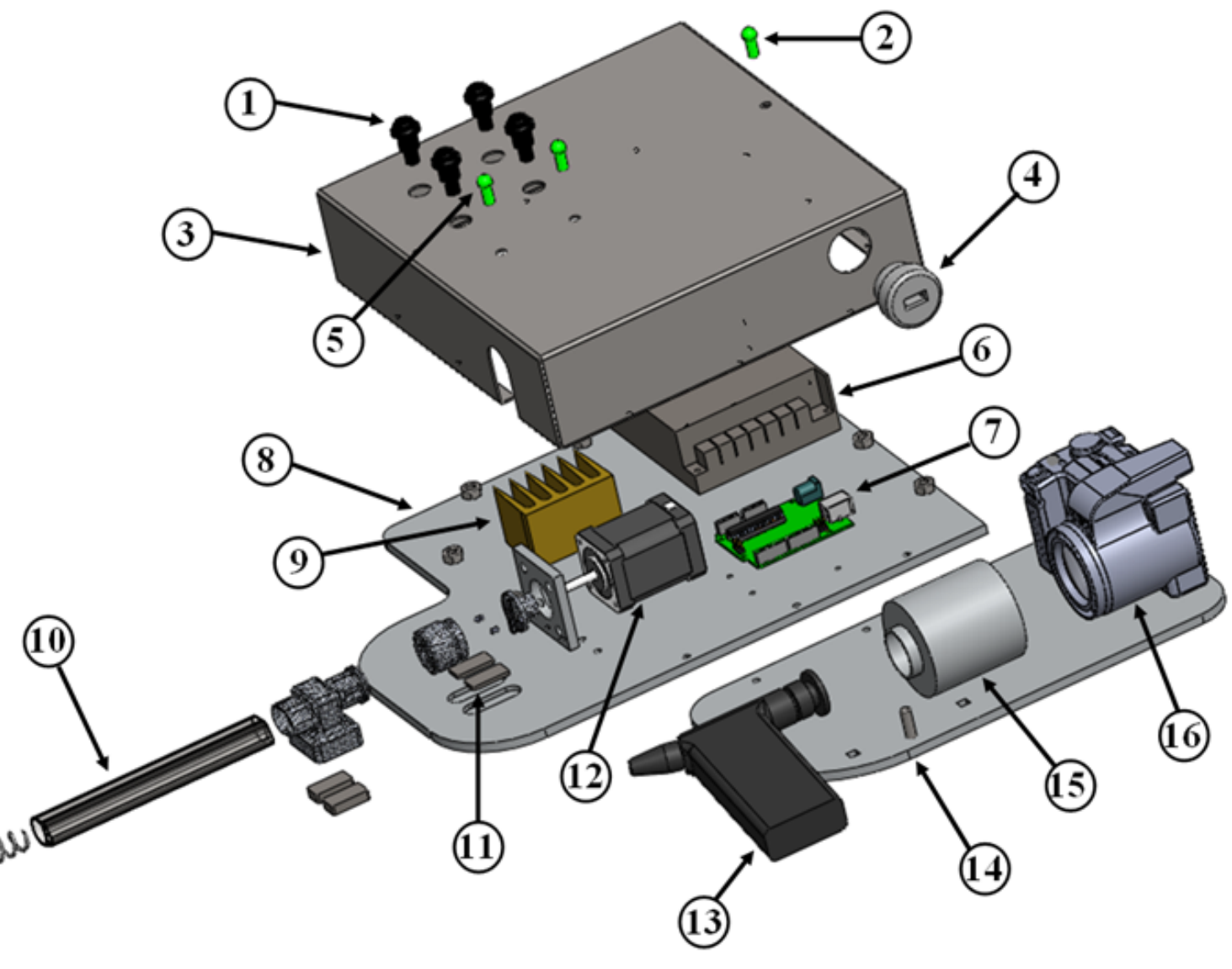




\section{Appendix I: Component Drawings}

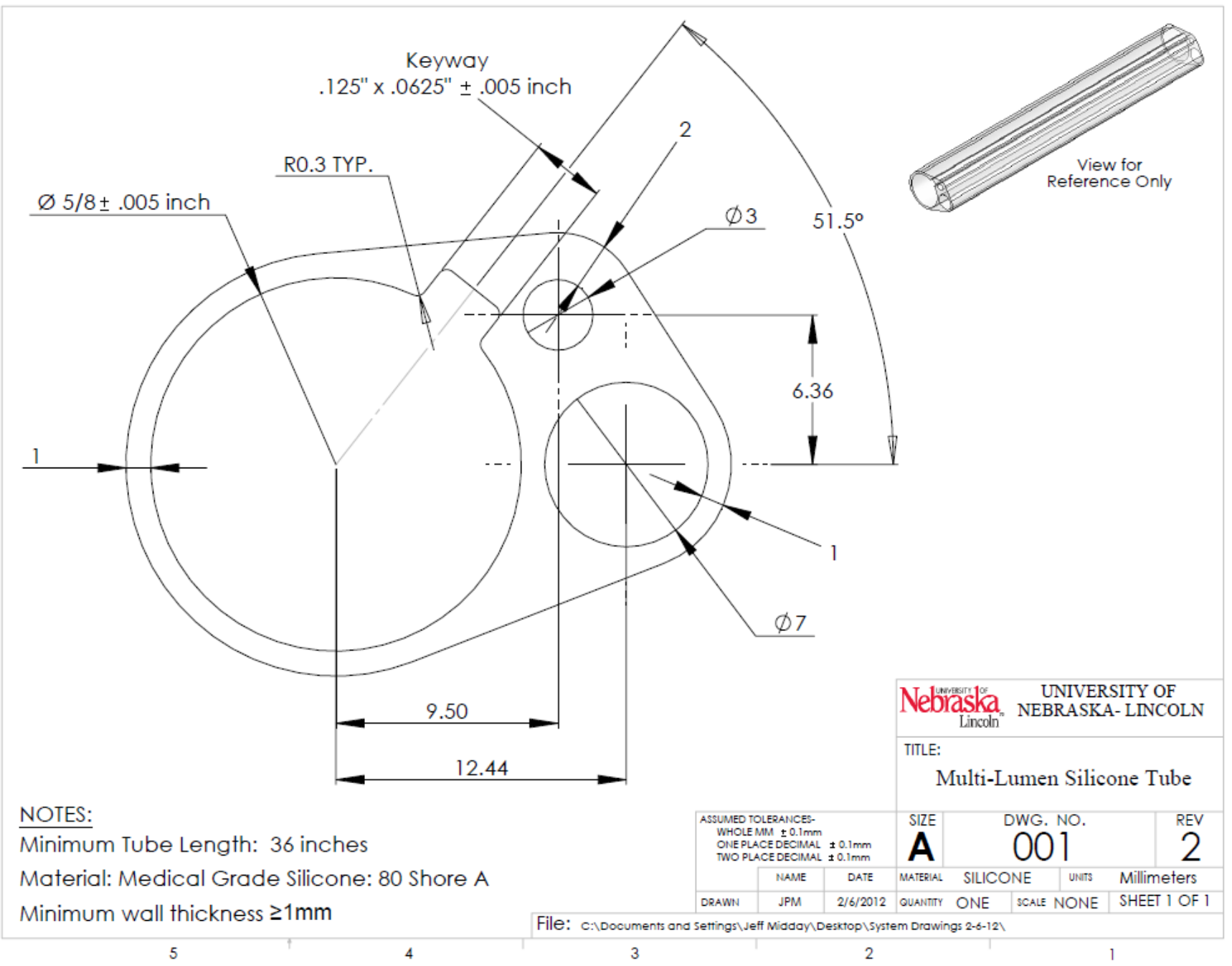




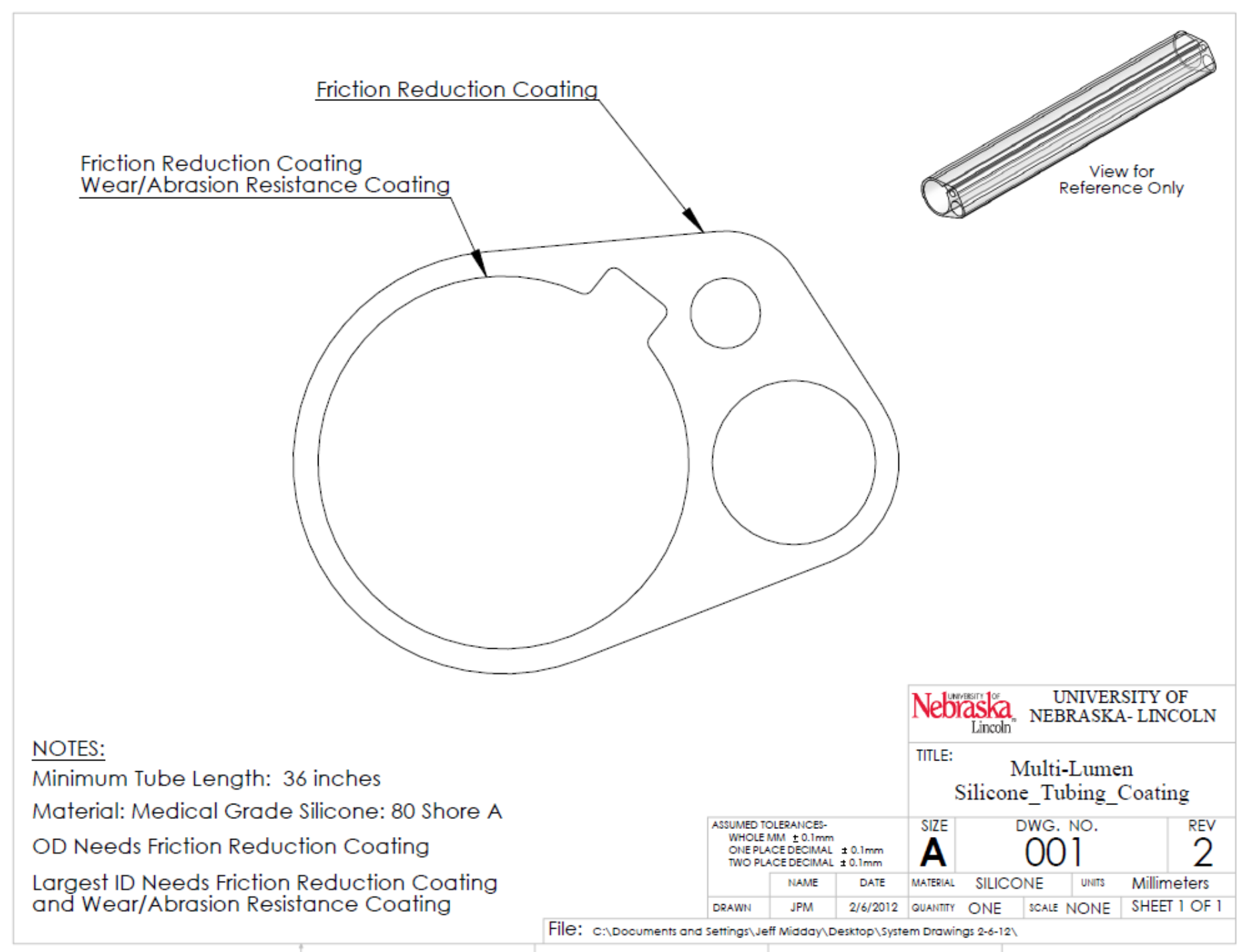

4

3 


\section{NOTES:}

-Helical Coil Compression Spring

-3 Coils per Inch

-Wire $\varnothing: 1 / 16 "$

-Finished Length: 42"

-End Condition: Open \& Ground

-Material: 304 Stainless Stee

*Rev2-Changes OD from $\varnothing 0.585$ to $\varnothing 0.560$
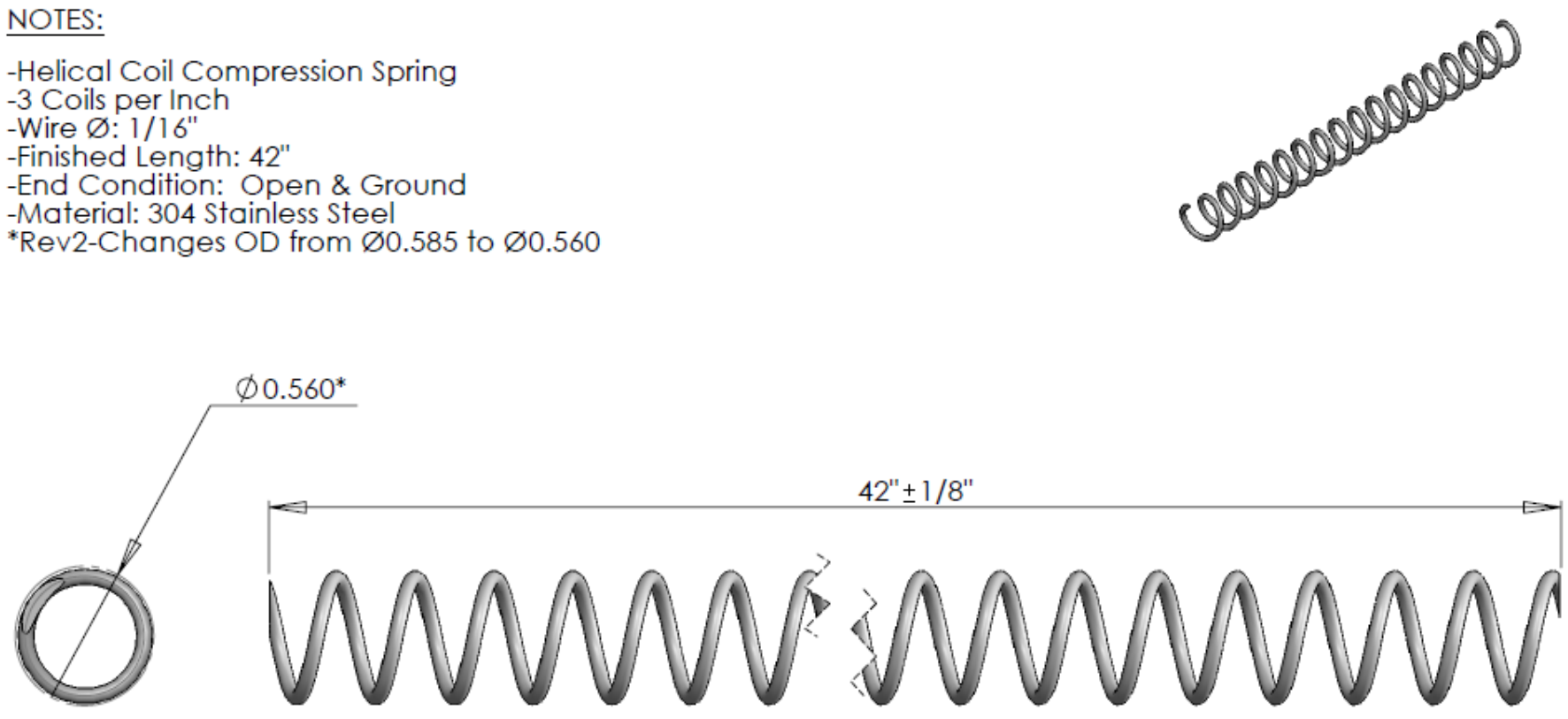

Nebraskia UNIVERSITY OF Lincoln $^{n}$ NEBRASKA-LINCOLN TITLE:

Drive Spring

\begin{tabular}{|c|c|c|c|c|c|c|}
\hline $\begin{array}{l}\text { SSUMED D } \\
\text { FRACT } \\
\text { TWO } \\
\text { THREE }\end{array}$ & & & $\stackrel{S I Z E}{\mathbf{A}}$ & DWG & & $2^{\text {REV }}$ \\
\hline & NAME & DATE & MATER/AL & 304 Stainless & UNTS & INCHES \\
\hline DRAWN & JPM & $2 / 6 / 2012$ & QUANATYY & \begin{tabular}{l|l}
5 & SCALE
\end{tabular} & NONE & SHEET 1 OF 1 \\
\hline
\end{tabular}
File: c:\Documents and Settings \Jeff Miaday Desktop \system Drawings 2-6-12 


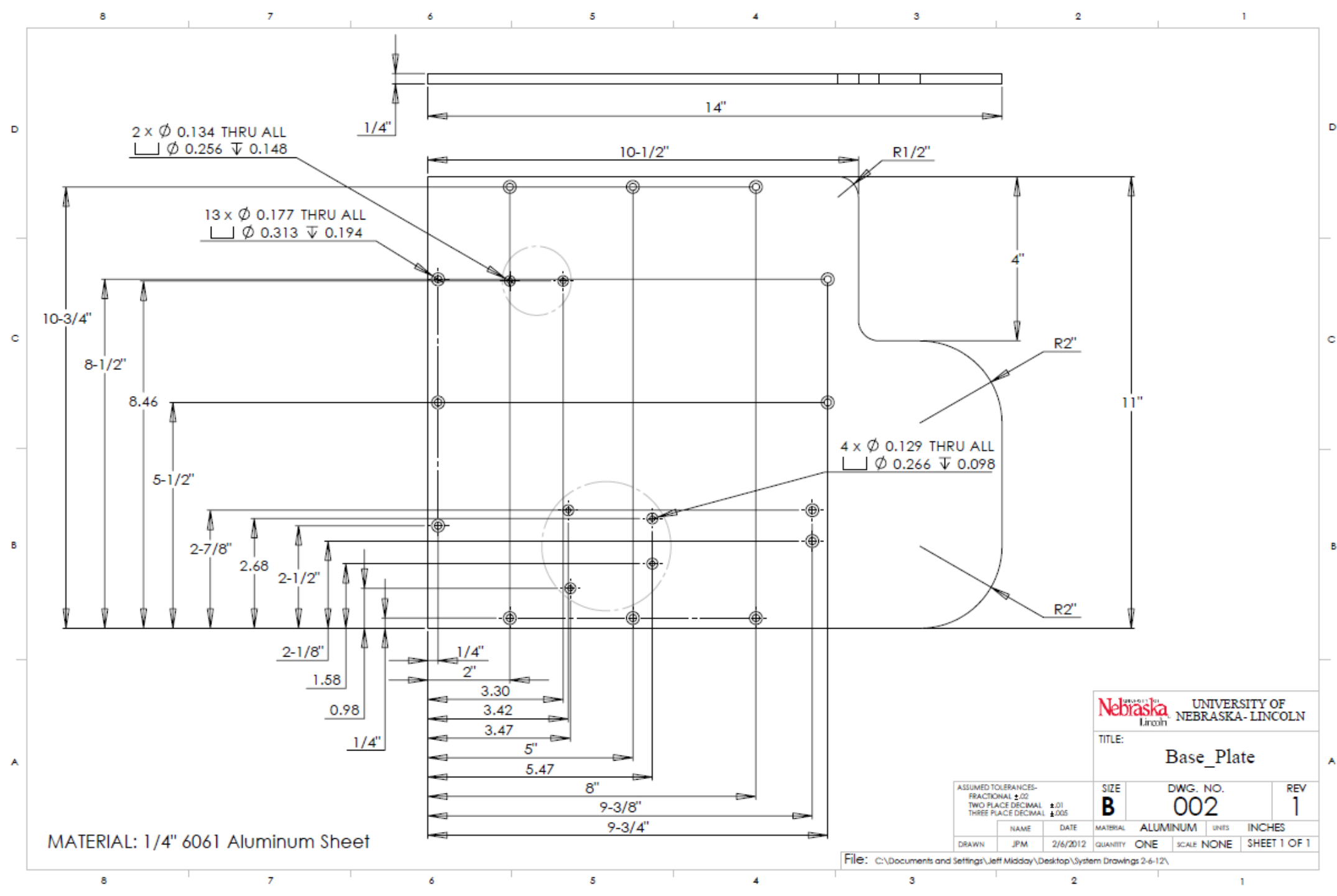




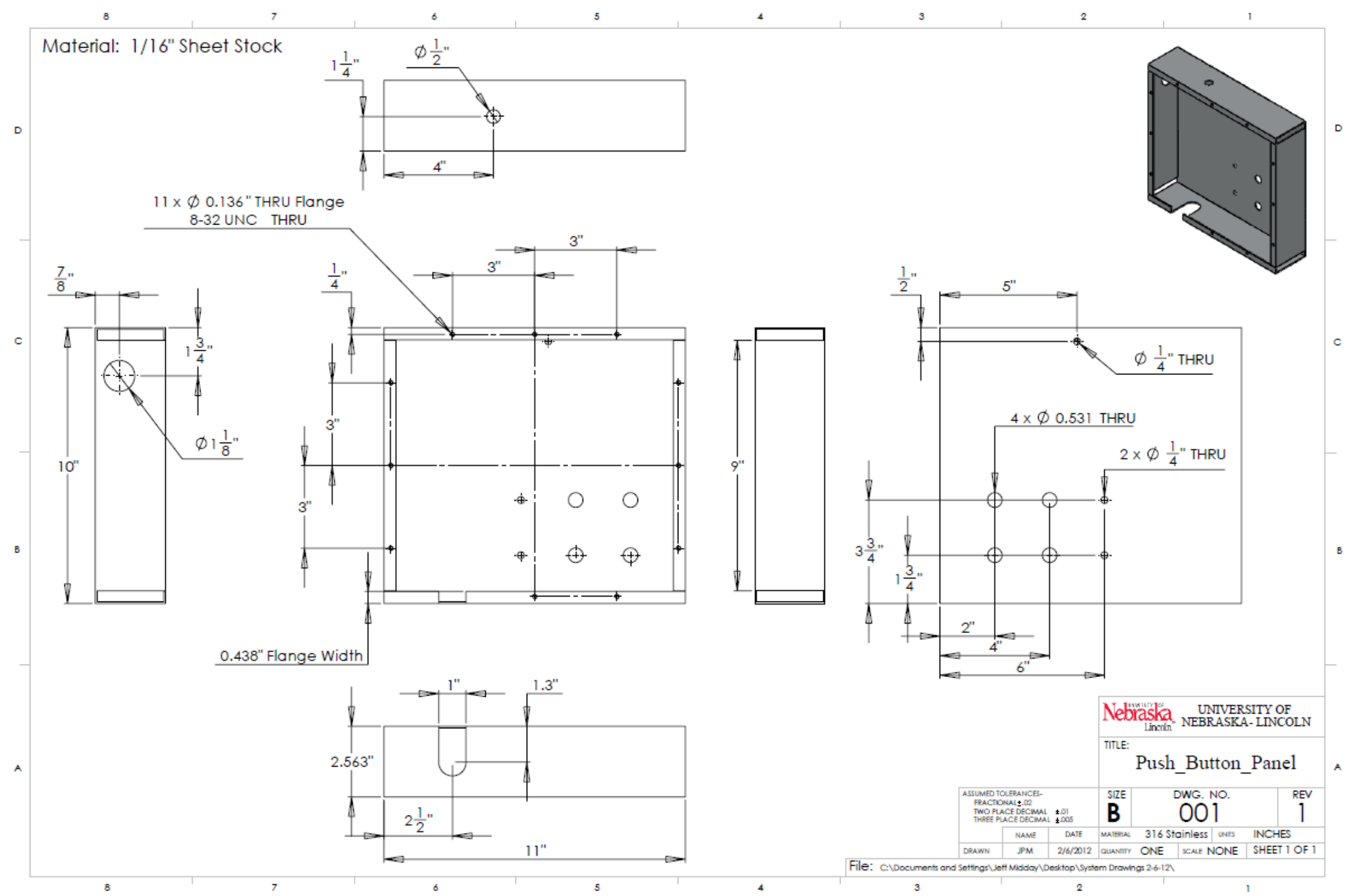




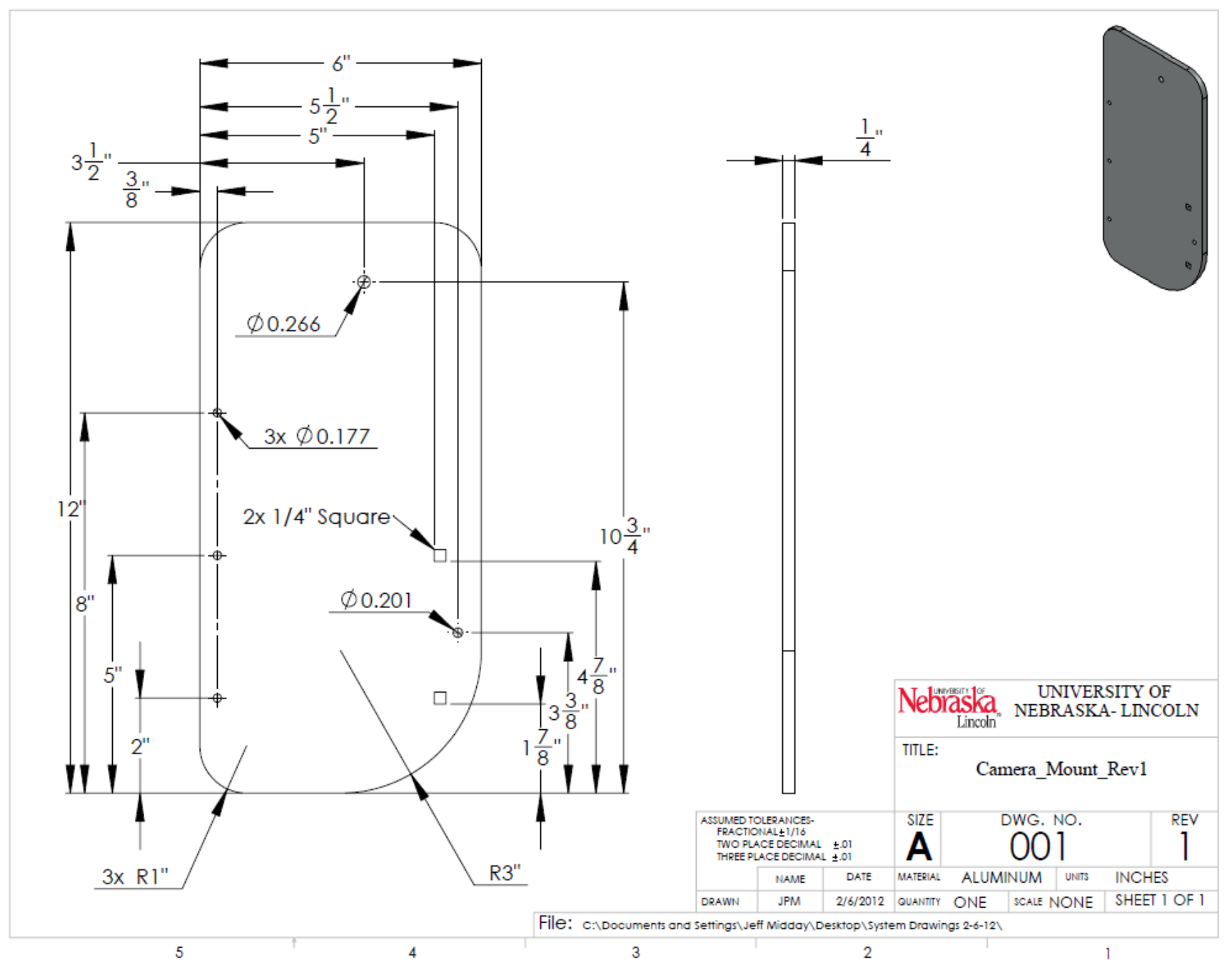

